\title{
Fast spectral algorithms from sum-of-squares proofs: tensor decomposition and planted sparse vectors
}

\author{
Samuel B. Hopkins ${ }^{*} \quad$ Tselil Schramm ${ }^{\dagger} \quad$ Jonathan Shi ${ }^{\ddagger} \quad$ David Steurer ${ }^{\S}$
}

February 4, 2016

\begin{abstract}
We consider two problems that arise in machine learning applications: the problem of recovering a planted sparse vector in a random linear subspace and the problem of decomposing a random low-rank overcomplete 3-tensor. For both problems, the best known guarantees are based on the sum-of-squares method. We develop new algorithms inspired by analyses of the sum-of-squares method. Our algorithms achieve the same or similar guarantees as sum-ofsquares for these problems but the running time is significantly faster.

For the planted sparse vector problem, we give an algorithm with running time nearly linear in the input size that approximately recovers a planted sparse vector with up to constant relative sparsity in a random subspace of $\mathbb{R}^{n}$ of dimension up to $\tilde{\Omega}(\sqrt{n})$. These recovery guarantees match the best known ones of Barak, Kelner, and Steurer (STOC 2014) up to logarithmic factors.

For tensor decomposition, we give an algorithm with running time close to linear in the input size (with exponent $\approx 1.086$ ) that approximately recovers a component of a random 3tensor over $\mathbb{R}^{n}$ of rank up to $\tilde{\Omega}\left(n^{4 / 3}\right)$. The best previous algorithm for this problem due to $\mathrm{Ge}$ and Ma (RANDOM 2015) works up to $\operatorname{rank} \tilde{\Omega}\left(n^{3 / 2}\right)$ but requires quasipolynomial time.
\end{abstract}

${ }^{*}$ Cornell University. samhop@cs . cornell . edu. Supported by an NSF Graduate Research Fellowship (NSF award no. 1144153) and by David Steurer's NSF CAREER award.

${ }^{\dagger}$ UC Berkeley, tschrammacs. berkeley. edu. Supported by an NSF Graduate Research Fellowship (NSF award no 1106400).

‡Cornell University, jshi@cs . cornell. edu. Supported by David Steurer's NSF CAREER award.

$\S$ Cornell University, dsteurer@cs . cornell. edu. Supported by a Microsoft Research Fellowship, a Alfred P. Sloan Fellowship, an NSF CAREER award, and the Simons Collaboration for Algorithms and Geometry. 


\section{Contents}

1 Introduction $\quad 1$

1.1 Planted sparse vector in random linear subspace . . . . . . . . . . . . . . . . . 2

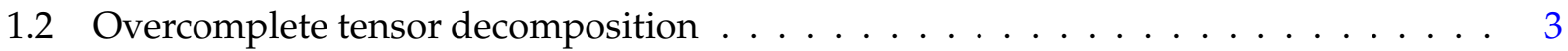

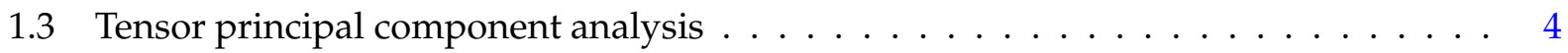

1.4 Related work . . . . . . . . . . . . . . . . . . . . 5

2 Techniques $\quad 6$

2.1 Planted sparse vector in random linear subspace . . . . . . . . . . . . . 8

2.2 Overcomplete tensor decomposition . . . . . . . . . . . . . . . 10

2.3 Tensor principal component analysis . . . . . . . . . . . . . . . . . 12

3 Preliminaries $\quad 13$

4 Planted sparse vector in random linear subspace $\quad 14$

4.1 Algorithm succeed on good basis . . . . . . . . . . . . . . . 16

4.2 Closeness of input basis and good basis . . . . . . . . . . . . . 18

5 Overcomplete tensor decomposition $\quad 20$

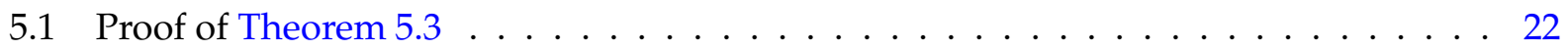

5.2 Spectral gap for diagonal terms: proof of Proposition $5.4 \ldots \ldots \ldots$

5.3 Bound for cross terms: proof of Proposition $5.5 \ldots \ldots \ldots \ldots$

5.4 Full algorithm and proof of Theorem $5.2 \ldots \ldots \ldots \ldots$. . . . . . . . . . 32

$6 \quad$ Tensor principal component analysis $\quad 37$

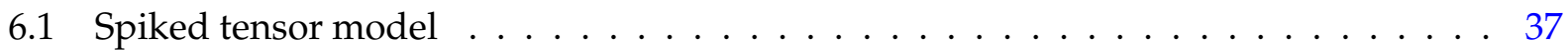

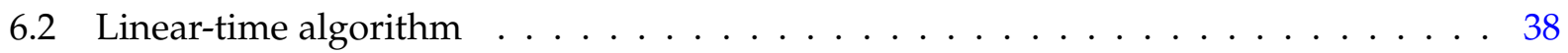

$\begin{array}{ll}\text { References } & 39\end{array}$

A Additional preliminaries $\quad 43$

A.1 Linear algebra . . . . . . . . . . . . . . . . . . . . . . . 43

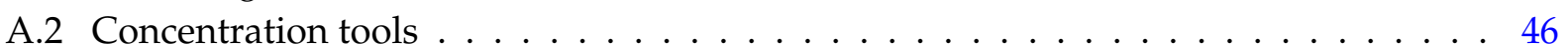

B Concentration bounds for planted sparse vector in random linear subspace 51

C Concentration bounds for overcomplete tensor decomposition 54

$\begin{array}{ll}\text { D Concentration bounds for tensor principal component analysis } & 61\end{array}$ 


\section{Introduction}

The sum-of-squares (SoS) method (also known as the Lasserre hierarchy) [Sho87, Par00, Nes00, Las01] is a powerful, semidefinite-programming based meta-algorithm that applies to a widerange of optimization problems. The method has been studied extensively for moderate-size polynomial optimization problems that arise for example in control theory and in the context of approximation algorithms for combinatorial optimization problems, especially constraint satisfaction and graph partitioning (see e.g. the survey [BS14]). For the latter, the SoS method captures and generalizes the best known approximation algorithms based on linear programming (LP), semidefinite programming (SDP), or spectral methods, and it is in many cases the most promising approach to obtain algorithms with better guarantees-especially in the context of Khot's Unique Games Conjecture $\left[\mathrm{BBH}^{+} 12\right]$.

A sequence of recent works applies the sum-of-squares method to basic problems that arise in unsupervised machine learning: in particular, recovering sparse vectors in linear subspaces and decomposing tensors in a robust way [BKS14, BKS15, HSS15, BM15, GM15]. For a wide range of parameters of these problems, SoS achieves significantly stronger guarantees than other methods, in polynomial or quasi-polynomial time.

Like other LP and SDP hierarchies, the sum-of-squares method comes with a degree parameter $d \in \mathbb{N}$ that allows for trading off running time and solution quality. This trade-off is appealing because for applications the additional utility of better solutions could vastly outweigh additional computational costs. Unfortunately, the computational cost grows rather steeply in terms of the parameter $d$ : the running time is $n^{O(d)}$ where $n$ is the number of variables (usually comparable to the instance size). Further, even when the SDP has size polynomial in the input (when $d=O(1)$ ), solving the underlying semidefinite programs is prohibitively slow for large instances.

In this work, we introduce spectral algorithms for planted sparse vector, tensor decomposition, and tensor principal components analysis (PCA) that exploit the same high-degree information as the corresponding sum-of-squares algorithms without relying on semidefinite programming, and achieve the same (or close to the same) guarantees. The resulting algorithms are quite simple (a couple of lines of матцAв code) and have considerably faster running times-quasi-linear or close to linear in the input size.

A surprising implication of our work is that for some problems, spectral algorithms can exploit information from larger values of the parameter $d$ without spending time $n^{O(d)}$. For example, one of our algorithms runs in nearly-linear time in the input size, even though it uses properties that the sum-of-squares method can only use for degree parameter $d \geqslant 4$. (In particular, the guarantees that the algorithm achieves are strictly stronger than the guarantees that SoS achieves for values of $d<4$.)

The initial successes of SoS in the machine learning setting gave hope that techniques developed in the theory of approximation algorithms, specifically the techniques of hierarchies of convex relaxations and rounding convex relaxations, could broadly impact the practice of machine

learning. This hope was dampened by the fact that in general, algorithms that rely on solving large semidefinite programs are too slow to be practical for the large-scale problems that arise in machine learning. Our work brings this hope back into focus by demonstrating for the first time that with some care SoS algorithms can be made practical for large-scale problems. 
In the following subsections we describe each of the problems that we consider, the prior best-known guarantee via the SoS hierarchy, and our results.

\subsection{Planted sparse vector in random linear subspace}

The problem of finding a sparse vector planted in a random linear subspace was introduced by Spielman, Wang, and Wright as a way of learning sparse dictionaries [SWW12]. Subsequent works have found further applications and begun studying the problem in its own right [DH14, BKS14, QSW14]. In this problem, we are given a basis for a $d$-dimensional linear subspace of $\mathbb{R}^{n}$ that is random except for one planted sparse direction, and the goal is to recover this sparse direction. The computational challenge is to solve this problem even when the planted vector is only mildly sparse (a constant fraction of non-zero coordinates) and the subspace dimension is large compared to the ambient dimension $\left(d \geqslant n^{\Omega(1)}\right)$.

Several kinds of algorithms have been proposed for this problem based on linear programming (LP), basic semidefinite programming (SDP), sum-of-squares, and non-convex gradient descent (alternating directions method).

An inherent limitation of simpler convex methods (LP and basic SDP) [SWW12, dGJL04] is that they require the relative sparsity of the planted vector to be polynomial in the subspace dimension (less than $n / \sqrt{d}$ non-zero coordinates).

Sum-of-squares and non-convex methods do not share this limitation. They can recover planted vectors with constant relative sparsity even if the subspace has polynomial dimension (up to dimension $O\left(n^{1 / 2}\right)$ for sum-of-squares [BKS14] and up to $O\left(n^{1 / 4}\right)$ for non-convex methods [QSW14]).

We state the problem formally:

Problem 1.1 (Planted sparse vector problem with ambient dimension $n \in \mathbb{N}$, subspace dimension $d \leqslant n$, sparsity $\varepsilon>0$, and accuracy $\eta>0$ ). Given an arbitrary orthogonal basis of a subspace spanned by vectors $v_{0}, v_{1}, \ldots, v_{d-1} \in \mathbb{R}^{n}$, where $v_{0}$ is a vector with at most $\varepsilon n$ non-zero entries and $v_{1}, \ldots, v_{d-1}$ are vectors sampled independently at random from the standard Gaussian distribution on $\mathbb{R}^{n}$, output a unit vector $v \in \mathbb{R}^{n}$ that has correlation $\left\langle v, v_{0}\right\rangle^{2} \geqslant 1-\eta$ with the sparse vector $v_{0}$.

Our Results. Our algorithm runs in nearly linear time in the input size, and matches the bestknown guarantees up to a polylogarithmic factor in the subspace dimension [BKS14].

Theorem 1.2 (Planted sparse vector in nearly-linear time). There exists an algorithm that, for every sparsity $\varepsilon>0$, ambient dimension $n$, and subspace dimension $d$ with $d \leqslant \sqrt{n} /(\log n)^{O(1)}$, solves the planted sparse vector problem with high probability for some accuracy $\eta \leqslant O\left(\varepsilon^{1 / 4}\right)+o_{n \rightarrow \infty}(1)$. The running time of the algorithm is $\tilde{O}(n d)$.

We give a technical overview of the proof in Section 2, and a full proof in Section 4.

Previous work also showed how to recover the planted sparse vector exactly. The task of going from an approximate solution to an exact one is a special case of standard compressed sensing (see e.g. [BKS14]). 
Table 1: Comparison of algorithms for the planted sparse vector problem with ambient dimension $n$, subspace dimension $d$, and relative sparsity $\varepsilon$.

\begin{tabular}{|c|c|c|c|c|}
\hline Reference & Technique & Runtime & Largest $d$ & Largest $\varepsilon$ \\
\hline \hline Demanet, Hand [DH14] & linear programming & poly & any & $\Omega(1 / \sqrt{d})$ \\
\hline Barak, Kelner, Steurer [BKS14] & SoS, general SDP & poly & $\Omega(\sqrt{n})$ & $\Omega(1)$ \\
\hline $\mathrm{Qu}$, Sun, Wright [QSW14] & alternating minimization & $\tilde{O}\left(n^{2} d^{5}\right)$ & $\Omega\left(n^{1 / 4}\right)$ & $\Omega(1)$ \\
\hline this work & SoS, partial traces & $\tilde{O}(n d)$ & $\Omega(\sqrt{n})$ & $\Omega(1)$ \\
\hline
\end{tabular}

\subsection{Overcomplete tensor decomposition}

Tensors naturally represent multilinear relationships in data. Algorithms for tensor decompositions have long been studied as a tool for data analysis across a wide-range of disciplines (see the early work of Harshman [Har70] and the survey [KB09]). While the problem is NPhard in the worst-case [Hås90, HL13], algorithms for special cases of tensor decomposition have recently led to new provable algorithmic results for several unsupervised learning problems [ $\mathrm{AGH}^{+} 14$, BCMV14, GVX14, AGHK14] including independent component analysis, learning mixtures of Gaussians [GHK15], Latent Dirichlet topic modeling [AFH $\left.{ }^{+} 15\right]$ and dictionary learning [BKS15]. Some previous learning algorithms can also be reinterpreted in terms of tensor decomposition [Cha96, MR06, NR09].

A key algorithmic challenge for tensor decompositions is overcompleteness, when the number of components is larger than their dimension (i.e., the components are linearly dependent). Most algorithms that work in this regime require tensors of order 4 or higher [LCC07, BCMV14]. For example, the FOOBI algorithm of [LCC07] can recover up to $\Omega\left(d^{2}\right)$ components given an order-4 tensor in dimension $d$ under mild algebraic independence assumptions for the componentssatisfied with high probability by random components. For overcomplete 3-tensors, which arise in many applications of tensor decompositions, such a result remains elusive.

Researchers have therefore turned to investigate average-case versions of the problem, when the components of the overcomplete 3-tensor are random: Given a 3-tensor $T \in \mathbb{R}^{d^{3}}$ of the form

$$
T=\sum_{i=1}^{n} a_{i} \otimes a_{i} \otimes a_{i},
$$

where $a_{1}, \ldots, a_{n}$ are random unit or Gaussian vectors, the goal is to approximately recover the components $a_{1}, \ldots, a_{n}$.

Algorithms based on tensor power iteration-a gradient-descent approach for tensor decomposition-solve this problem in polynomial time when $n \leqslant C \cdot d$ for any constant $C \geqslant 1$ (the running time is exponential in $C$ ) [AGJ15]. Tensor power iteration also admits local convergence analyses for up to $n \leqslant \tilde{\Omega}\left(d^{1.5}\right)$ components [AGJ15, AGJ14]. Unfortunately, these analyses do not give polynomial-time algorithms because it is not known how to efficiently obtain the kind of initializations assumed by the analyses.

Recently, Ge and Ma [GM15] were able to show that a tensor-decomposition algorithm [BKS15] based on sum-of-squares solves the above problem for $n \leqslant \tilde{\Omega}\left(d^{1.5}\right)$ in quasi-polynomial time $n^{O(\log n)}$. The key ingredient of their elegant analysis is a subtle spectral concentration bound for a particular degree-4 matrix-valued polynomial associated with the decomposition problem of random 
Table 2: Comparison of decomposition algorithms for overcomplete 3-tensors with $n$ components in dimension $d$.

\begin{tabular}{|c|c|c|c|c|}
\hline Reference & Technique & Runtime & Largest $n$ & Components \\
\hline \hline Anandkumar et al. [AGJ15] & tensor power iteration & poly & $C \cdot d$ & incoherent \\
\hline Ge, Ma [GM15] & SoS, general SDP & $n^{O(\log n)}$ & $\tilde{\Omega}\left(d^{3 / 2}\right)$ & $\mathcal{N}\left(0, \frac{1}{d} \mathrm{Id}_{d}\right)$ \\
\hline this work $^{\mathrm{b}}$ & SoS, partial traces & $\tilde{O}\left(n d^{1+\omega}\right)$ & $\tilde{\Omega}\left(d^{4 / 3}\right)$ & $\mathcal{N}\left(0, \frac{1}{d} \mathrm{Id}_{d}\right)$ \\
\hline
\end{tabular}

a The analysis shows that for every constant $C \geqslant 1$, the running time is polynomial for $n \leqslant C \cdot d$ components. The analysis assumes that the components also satisfy other random-like properties besides incoherence.

${ }^{\mathrm{b}}$ Here, $\omega \leqslant 2.373$ is the constant so that $d \times d$ matrices can be multiplied in $O\left(d^{\omega}\right)$ arithmetic operations.

overcomplete 3-tensors.

We state the problem formally:

Problem 1.3 (Random tensor decomposition with dimension $d$, rank $n$, and accuracy $\eta$ ). Let $a_{1}, \ldots, a_{n} \in \mathbb{R}^{d}$ be independently sampled vectors from the Gaussian distribution $\mathcal{N}\left(0, \frac{1}{d} \operatorname{Id}_{d}\right)$, and let $\mathbf{T} \in\left(\mathbb{R}^{d}\right)^{\otimes 3}$ be the 3-tensor $\mathbf{T}=\sum_{i=1}^{n} a_{i}^{\otimes 3}$.

Single component: Given $\mathbf{T}$ sampled as above, find a unit vector $b$ that has correlation $\max _{i}\left\langle a_{i}, b\right\rangle \geqslant 1-\eta$ with one of the vectors $a_{i}$.

All components: Given $\mathbf{T}$ sampled as above, find a set of unit vectors $\left\{b_{1}, \ldots, b_{n}\right\}$ such that $\left\langle a_{i}, b_{i}\right\rangle \geqslant 1-\eta$ for every $i \in[n]$.

Our Results. We give the first polynomial-time algorithm for decomposing random overcomplete 3-tensors with up to $\omega(d)$ components. Our algorithms works as long as the number of components satisfies $n \leqslant \tilde{\Omega}\left(d^{4 / 3}\right)$, which comes close to the bound $\tilde{\Omega}\left(d^{1.5}\right)$ achieved by the aforementioned quasi-polynomial algorithm of Ge and Ma. For the single-component version of the problem, our algorithm runs in time close to linear in the input size.

Theorem 1.4 (Fast random tensor decomposition). There exist randomized algorithms that, for every dimension $d$ and rank $n$ with $d \leqslant n \leqslant d^{4 / 3} /(\log n)^{O(1)}$, solve the random tensor decomposition problem with probability $1-o(1)$ for some accuracy $\eta \leqslant \tilde{O}\left(n^{3} / d^{4}\right)^{1 / 2}$. The running time for the single-component version of the problem is $\tilde{O}\left(\min \left\{d^{1+\omega}, d^{3.257}\right\}\right)$, where $d^{\omega}$ is the time to multiply two $d$-by-d matrices. The running time for the all-components version of the problem is $\tilde{O}\left(n \cdot \min \left\{d^{1+\omega}, d^{3.257}\right\}\right)$.

We give a technical overview of the proof in Section 2, and a full proof in Section 5.

We remark that the above algorithm only requires access to the input tensor with some fixed inverse polynomial accuracy because each of its four steps amplifies errors by at most a polynomial factor (see Algorithm 5.17). In this sense, the algorithm is robust.

\subsection{Tensor principal component analysis}

The problem of tensor principal component analysis is similar to the tensor decomposition problem. However, here the focus is not on the number of components in the tensor, but about recovery in the presence of a large amount of random noise. We are given as input a tensor $\tau \cdot v^{\otimes 3}+\mathbf{A}$, where $v \in \mathbb{R}^{n}$ is a unit vector and the entries of $\mathbf{A}$ are chosen iid from $\mathcal{N}(0,1)$. This spiked tensor model was introduced by Montanari and Richard [RM14], who also obtained the first algorithms 
Table 3: Comparison of algorithms for principal component analysis of 3-tensors in dimension $d$ and with signal-to-noise ratio $\tau$.

\begin{tabular}{|c|c|c|c|}
\hline Reference & Technique & Runtime & Smallest $\tau$ \\
\hline \hline Montanari, Richard [RM14] & spectral & $\tilde{O}\left(d^{3}\right)$ & $n$ \\
\hline Hopkins, Shi, Steurer [HSS15] & SoS, spectral & $\tilde{O}\left(d^{3}\right)$ & $\Omega\left(n^{3 / 4}\right)$ \\
\hline this work & SoS, partial traces & $O\left(d^{3}\right)$ & $\tilde{\Omega}\left(n^{3 / 4}\right)$ \\
\hline
\end{tabular}

to solve the model with provable statistical guarantees. The spiked tensor model was subsequently addressed by a subset of the present authors [HSS15], who applied the SoS approach to improve the signal-to-noise ratio required for recovery from odd-order tensors.

We state the problem formally:

Problem 1.5 (Tensor principal components analysis with signal-to-noise ratio $\tau$ and accuracy $\eta$ ). Let $\mathbf{T} \in\left(\mathbb{R}^{d}\right)^{\otimes 3}$ be a tensor so that $\mathbf{T}=\tau \cdot v^{\otimes 3}+A$, where $A$ is a tensor with independent standard gaussian entries and $v \in \mathbb{R}^{d}$ is a unit vector. Given $\mathbf{T}$, recover a unit vector $v^{\prime} \in \mathbb{R}^{d}$ such that $\left\langle v^{\prime}, v\right\rangle \geqslant 1-\eta$.

Our results. For this problem, our improvements over the previous results are more modest—we achieve signal-to-noise guarantees matching [HSS15], but with an algorithm that runs in linear time rather than near-linear time (time $O\left(d^{3}\right)$ rather than $O\left(d^{3}\right.$ polylog $\left.d\right)$, for an input of size $\left.d^{3}\right)$.

Theorem 1.6 (Tensor principal component analysis in linear time). There is an algorithm which solves the tensor principal component analysis problem with accuracy $\eta>0$ whenever the signal-to-noise ratio satisfies $\tau \geqslant O\left(n^{3 / 4} / \eta \cdot \log ^{1 / 2} n\right)$. Furthermore, the algorithm runs in time $O\left(d^{3}\right)$.

Though for tensor PCA our improvement over previous work is modest, we include the results here as this problem is a pedagogically poignant illustration of our techniques. We give a technical overview of the proof in Section 2, and a full proof in Section 6.

\subsection{Related work}

Foremost, this work builds upon the SoS algorithms of [BKS14, BKS15, GM15, HSS15]. In each of these previous works, a machine learning decision problem is solved using an SDP relaxation for SoS. In these works, the SDP value is large in the yEs case and small in the No case, and the SDP value can be bounded using the spectrum of a specific matrix. This was implicit in [BKS14, BKS15], and in [HSS15] it was used to obtain a fast algorithm as well. In our work, we design spectral algorithms which use smaller matrices, inspired by the SoS certificates in previous works, to solve these machine-learning problems much faster, with almost matching guarantees.

A key idea in our work is that given a large matrix with information encoded in the matrix's spectral gap, one can often efficiently "compress" the matrix to a much smaller one without losing that information. This is particularly true for problems with planted solutions. In this way, we are able to improve running time by replacing an $n^{O(d)}$-sized SDP with an eigenvector computation for an $n^{k} \times n^{k}$ matrix, for some $k<d$. 
The idea of speeding up LP and SDP hierarchies for specific problems has been investigated in a series of previous works [dIVK07, BRS11, GS12], which shows that with respect to local analyses of the sum-of-squares algorithm it is sometimes possible to improve the running time from $n^{O(d)}$ to $2^{O(d)} \cdot n^{O(1)}$. However, the scopes and strategies of these works are completely different from ours. First, the notion of local analysis from these works does not apply to the problems considered here. Second, these works employ the ellipsoid method with a separation oracle inspired by rounding algorithms, whereas we reduce the problem to an ordinary eigenvector computation.

It would also be interesting to see if our methods can be used to speed up some of the other recent successful applications of SoS to machine-learning type problems, such as [BM15], or the application of [BKS14] to tensor decomposition with components that are well-separated (rather than random). Finally, we would be remiss not to mention that SoS lower bounds exist for several of these problems, specifically for tensor principal components analysis, tensor prediction, and sparse PCA [HSS15, BM15, MW15]. The lower bounds in the SoS framework are a good indication that we cannot expect spectral algorithms achieving better guarantees.

\section{Techniques}

Sum-of-squares method (for polynomial optimization over the sphere). The problems we consider are connected to optimization problems of the following form: Given a homogeneous $n$ variate real polynomial $f$ of constant degree, find a unit vector $x \in \mathbb{R}^{n}$ so as to maximize $f(x)$. The sum-of-squares method allows us to efficiently compute upper bounds on the maximum value of such a polynomial $f$ over the unit sphere.

For the case that $k=\operatorname{deg}(f)$ is even, the most basic upper bound of this kind is the largest eigenvalue of a matrix representation of $f$. A matrix representation of a polynomial $f$ is a symmetric matrix $M$ with rows and columns indexed by monomials of degree $k / 2$ so that $f(x)$ can be written as the quadratic form $f(x)=\left\langle x^{\otimes k / 2}, M x^{\otimes k / 2}\right\rangle$, where $x^{\otimes k / 2}$ is the $k / 2$-fold tensor power of $x$. The largest eigenvalue of a matrix representation $M$ is an upper bound on the value of $f(x)$ over unit $x \in \mathbb{R}^{n}$ because

$$
f(x)=\left\langle x^{\otimes k / 2}, M x^{\otimes k / 2}\right\rangle \leqslant \lambda_{\max }(M) \cdot\left\|x^{\otimes k / 2}\right\|_{2}^{2}=\lambda_{\max }(M) .
$$

The sum-of-squares methods improves on this basic spectral bound systematically by associating a large family of polynomials (potentially of degree higher than $\operatorname{deg}(f)$ ) with the input polynomial $f$ and computing the best possible spectral bound within this family of polynomials. Concretely, the sum-of-squares method with degree parameter $d$ applied to a polynomial $f$ with $\operatorname{deg}(f) \leqslant d$ considers the affine subspace of polynomials $\left\{f+\left(1-\|x\|_{2}^{2}\right) \cdot g \mid \operatorname{deg}(g) \leqslant d-2\right\} \subseteq \mathbb{R}[x]$ and minimizes $\lambda_{\max }(M)$ among all matrix representations ${ }^{1} M$ of polynomials in this space. ${ }^{2}$ The problem of searching through this affine linear space of polynomials and their matrix represen-

\footnotetext{
${ }^{1}$ Earlier we defined matrix representations only for homogeneous polynomials of even degree. In general, a matrix representation of a polynomial $g$ is a symmetric matrix $M$ with rows and columns indexed by monomials of degree at most $\ell=\operatorname{deg}(g) / 2$ such that $g(x)=\left\langle x^{\otimes \leqslant \ell}, M x^{\otimes \leqslant \ell}\right\rangle$ (as a polynomial identity), where $x^{\otimes \leqslant \ell}=\frac{1}{\sqrt{\ell+1}}\left(x^{\otimes 0}, x^{\otimes 1}, \ldots, x^{\otimes \ell}\right)$ is the vector of all monomials of degree at most $\ell$. Note that $\left\|x^{\otimes \leqslant \ell}\right\|=1$ for all $x$ with $\|x\|=1$.

2 The name of the method stems from the fact that this last step is equivalent to finding the minimum number $\lambda$ such that the space contains a polynomial of the form $\lambda-\left(g_{1}^{2}+\cdots+g_{t}^{2}\right)$, where $g_{1}, \ldots, g_{t}$ are polynomials of degree at most $d / 2$.
} 
tations and finding the one of smallest maximum eigenvalue can be solved using semidefinite programming.

Our approach for faster algorithms based on SoS algorithms is to construct specific matrices (polynomials) in this affine linear space, then compute their top eigenvectors. By designing our matrices carefully, we ensure that our algorithms have access to the same higher degree information that the sum-of-squares algorithm can access, and this information affords an advantage over the basic spectral methods for these problems. At the same time, our algorithms avoid searching for the best polynomial and matrix representation, which gives us faster running times since we avoid semidefinite programming. This approach is well suited to average-case problems where we avoid the problem of adversarial choice of input; in particular it is applicable to machine learning problems where noise and inputs are assumed to be random.

Compressing matrices with partial traces. A serious limitation of the above approach is that the representation of a degree- $d$ polynomial requires size roughly $n^{d}$. Hence, even avoiding the use of semidefinite programming, improving upon running time $O\left(n^{d}\right)$ requires additional ideas.

In each of the problems that we consider, we have a large matrix (suggested by a SoS algorithm) with a "signal" planted in some amount of "noise". We show that in some situations, this large matrix can be compressed significantly without loss in the signal by applying partial trace operations. In these situations, the partial trace yields a smaller matrix with the same signal-to-noise ratio as the large matrix suggested by the SoS algorithm, even in situations when lower degree sum-of-squares approaches are known to fail.

The partial trace $\operatorname{Tr}_{\mathbb{R}^{d}}: \mathbb{R}^{d^{2} \times d^{2}} \rightarrow \mathbb{R}^{d \times d}$ is the linear operator that satisfies $\operatorname{Tr}_{\mathbb{R}^{d}} A \otimes B=(\operatorname{Tr} A) \cdot B$ for all $A, B \in \mathbb{R}^{d \times d}$. To see how the partial trace can be used to compress large matrices to smaller ones with little loss, consider the following problem: Given a matrix $M \in \mathbb{R}^{d^{2} \times d^{2}}$ of the form $M=\tau \cdot(v \otimes v)(v \otimes v)^{\top}+A \otimes B$ for some unit vector $v \in \mathbb{R}^{d}$ and matrices $A, B \in \mathbb{R}^{d \times d}$, we wish to recover the vector $v$. (This is a simplified version of the planted problems that we consider in this paper, where $\tau \cdot(v \otimes v)(v \otimes v)^{\top}$ is the signal and $A \otimes B$ plays the role of noise.)

It is straightforward to see that the matrix $A \otimes B$ has spectral norm $\|A \otimes B\|=\|A\| \cdot\|B\|$, and so when $\tau \gg\|A\|\|B\|$, the matrix $M$ has a noticeable spectral gap, and the top eigenvector of $M$ will be close to $v \otimes v$. If $|\operatorname{Tr} A| \approx\|A\|$, the matrix $\operatorname{Tr}_{\mathbb{R}^{d}} M=\tau \cdot v v^{\top}+\operatorname{Tr}(A) \cdot B$ has a matching spectral gap, and we can still recover $v$, but now we only need to compute the top eigenvector of a $d \times d$ (as opposed to $\left.d^{2} \times d^{2}\right)$ matrix. ${ }^{3}$

If $A$ is a Wigner matrix (e.g. a symmetric matrix with iid \pm 1 entries), then both $\operatorname{Tr}(A),\|A\| \approx \sqrt{n}$, and the above condition is indeed met. In our average case/machine learning settings the "noise" component is not as simple as $A \otimes B$ with $A$ a Wigner matrix. Nonetheless, we are able to ensure that the noise displays a similar behavior under partial trace operations. In some cases, this requires additional algorithmic steps, such as random projection in the case of tensor decomposition, or centering the matrix eigenvalue distribution in the case of the planted sparse vector.

It is an interesting question if there are general theorems describing the behavior of spectral norms under partial trace operations. In the current work, we compute the partial traces explicitly and estimate their norms directly. Indeed, our analyses boil down to concentrations bounds for

\footnotetext{
${ }^{3}$ In some of our applications, the matrix $M$ is only represented implicitly and has size super linear in the size of the input, but nevertheless we can compute the top eigenvector of the partial trace $\operatorname{Tr}_{\mathbb{R}^{d}} M$ in nearly-linear time.
} 
special matrix polynomials. A general theory for the concentration of matrix polynomials is a notorious open problem (see [MW13]).

Partial trace operations have previously been applied for rounding SoS relaxations. Specifically, the operation of reweighing and conditioning, used in rounding algorithms for sum-of-squares such as [BRS11, RT12, BKS14, BKS15, LR15], corresponds to applying a partial trace operation to the moments matrix returned by the sum-of-squares relaxation.

We now give a technical overview of our algorithmic approach for each problem, and some broad strokes of the analysis for each case. Our most substantial improvements in runtime are for the planted sparse vector and overcomplete tensor decomposition problems (Section 2.1 and Section 2.2 respectively). Our algorithm for tensor PCA is the simplest application of our techniques, and it may be instructive to skip ahead and read about tensor PCA first (Section 2.3).

\subsection{Planted sparse vector in random linear subspace}

Recall that in this problem we are given a linear subspace $U$ (represented by some basis) that is spanned by a $k$-sparse unit vector $v_{0} \in \mathbb{R}^{d}$ and random unit vectors $v_{1}, \ldots, v_{d-1} \in \mathbb{R}^{d}$. The goal is to recover the vector $v_{0}$ approximately.

Background and SoS analysis. Let $A \in \mathbb{R}^{n \times d}$ be a matrix whose columns form an orthonormal basis for $U$. Our starting point is the polynomial $f(x)=\|A x\|_{4}^{4}=\sum_{i=1}^{n}(A x)_{i}^{4}$. Previous work showed that for $d \ll \sqrt{n}$ the maximizer of this polynomial over the sphere corresponds to a vector close to $v_{0}$ and that degree- 4 sum-of-squares is able to capture this fact $\left[\mathrm{BBH}^{+} 12, \mathrm{BKS} 14\right]$. Indeed, typical random vectors $v$ in $\mathbb{R}^{n}$ satisfy $\|v\|_{4}^{4} \approx 1 / n$ whereas our planted vector satisfies $\left\|v_{0}\right\|_{4}^{4} \geqslant 1 / k \gg 1 / n$, and this degree- 4 information is leveraged by the SoS algorithms.

The polynomial $f$ has a convenient matrix representation $M=\sum_{i=1}^{n}\left(a_{i} a_{i}^{\top}\right)^{\otimes 2}$, where $a_{1}, \ldots, a_{n}$ are the rows of the generator matrix $A$. It turns out that the eigenvalues of this matrix indeed give information about the planted sparse vector $v_{0}$. In particular, the vector $x_{0} \in \mathbb{R}^{d}$ with $A x_{0}=v_{0}$ witnesses that $M$ has an eigenvalue of at least $1 / k$ because $M^{\prime}$ s quadratic form with the vector $x_{0}^{\otimes 2}$ satisfies $\left\langle x_{0}^{\otimes 2}, M x_{0}^{\otimes 2}\right\rangle=\left\|v_{0}\right\|_{4}^{4} \geqslant 1 / k$. If we let $M^{\prime}$ be the corresponding matrix for the subspace $U$ without the planted sparse vector, $M^{\prime}$ turns out to have only eigenvalues of at most $O(1 / n)$ up to a single spurious eigenvalue with eigenvector far from any vector of the form $x \otimes x\left[\mathrm{BBH}^{+} 12\right]$.

It follows that in order to distinguish between a random subspace with a planted sparse vector (YES case) and a completely random subspace (No case), it is enough to compute the second-largest eigenvalue of a $d^{2}$-by- $d^{2}$ matrix (representing the 4-norm polynomial over the subspace as above). This decision version of the problem, while strictly speaking easier than the search version above, is at the heart of the matter: one can show that the large eigenvalue for the yEs case corresponds to an eigenvector which encodes the coefficients of the sparse planted vector in the basis.

Improvements. The best running time we can hope for with this basic approach is $O\left(d^{4}\right)$ (the size of the matrix). Since we are interested in $d \leqslant O(\sqrt{n})$, the resulting running time $O\left(n d^{2}\right)$ would be subquadratic but still super-linear in the input size $n \cdot d$ (for representing a $d$-dimensional subspace of $\mathbb{R}^{n}$ ). To speed things up, we use the partial trace approach outlined above. We will begin by applying the partial trace approach naively, obtaining reasonable bounds, and then show that a small modification to the matrix before the partial trace operation allows us to achieve even smaller signal-to-noise ratios. 
In the planted case, we may approximate $M \approx \frac{1}{k}\left(x_{0} x_{0}^{\top}\right)^{\otimes 2}+Z$, where $x_{0}$ is the vector of coefficients of $v_{0}$ in the basis representation given by $A$ (so that $A x_{0}=v_{0}$ ), and $Z$ is the noise matrix. Since $\left\|x_{0}\right\|=$ 1 , the partial trace operation preserves the projector $\left(x_{0} x_{0}^{\top}\right)^{\otimes 2}$ in the sense that $\operatorname{Tr}_{\mathbb{R}^{d}}\left(x_{0} x_{0}^{\top}\right)^{\otimes 2}=x_{0} x_{0}^{\top}$. Hence, with our heuristic approximation for $M$ above, we could show that the top eigenvector of $\operatorname{Tr}_{\mathbb{R}^{d}} M$ is close to $x_{0}$ by showing that the spectral norm bound $\left\|\operatorname{Tr}_{\mathbb{R}^{d}} Z\right\| \leqslant o(1 / k)$.

The partial trace of our matrix $M=\sum_{i=1}^{n}\left(a_{i} a_{i}^{\top}\right) \otimes\left(a_{i} a_{i}^{\top}\right)$ is easy to compute directly,

$$
N=\operatorname{Tr}_{\mathbb{R}^{d}} M=\sum_{i=1}^{n}\left\|a_{i}\right\|_{2}^{2} \cdot a_{i} a_{i}^{\top} .
$$

In the yEs case (random subspace with planted sparse vector), a direct computation shows that

$$
\lambda_{\mathrm{YES}} \geqslant\left\langle x_{0}, N x_{0}\right\rangle \approx \frac{d}{n} \cdot\left(1+\frac{n}{d}\left\|v_{0}\right\|_{4}^{4}\right) \geqslant \frac{d}{n}\left(1+\frac{n}{d k}\right) .
$$

Hence, a natural approach to distinguish between the yes case and No case (completely random subspace) is to upper bound the spectral norm of $N$ in the no case.

In order to simplify the bound on the spectral norm of $N$ in the No case, suppose that the columns of $A$ are iid samples from the Gaussian distribution $\mathcal{N}\left(0, \frac{1}{d}\right.$ Id) (rather than an orthogonal basis for the random subspace)-Lemma 4.6 establishes that this simplification is legitimate. In this simplified setup, the matrix $N$ in the No case is the sum of $n$ iid matrices $\left\{\left\|a_{i}\right\|^{2} \cdot a_{i} a_{i}^{\top}\right\}$, and we can upper bound its spectral norm $\lambda_{\mathrm{No}}$ by $d / n \cdot(1+O(\sqrt{d / n}))$ using standard matrix concentration bounds. Hence, using the spectral norm of $N$, we will be able to distinguish between the yes case and the no case as long as

$$
\sqrt{d / n} \ll n /(d k) \Longrightarrow \lambda_{\mathrm{NO}} \ll \lambda_{\mathrm{YES}}
$$

For linear sparsity $k=\varepsilon \cdot n$, this inequality is true so long as $d \ll\left(n / \varepsilon^{2}\right)^{1 / 3}$, which is somewhat worse than the bound $\sqrt{n}$ bound on the dimension that we are aiming for.

Recall that $\operatorname{Tr} B=\sum_{i} \lambda_{i}(B)$ for a symmetric matrix $B$. As discussed above, the partial trace approach works best when the noise behaves as the tensor of two Wigner matrices, in that there are cancellations when the eigenvalues of the noise are summed. In our case, the noise terms $\left(a_{i} a_{i}^{\top}\right) \otimes\left(a_{i} a_{i}^{\top}\right)$ do not have this property, as in fact $\operatorname{Tr} a_{i} a_{i}^{\top}=\left\|a_{i}\right\|^{2} \approx d / n$. Thus, in order to improve the dimension bound, we will center the eigenvalue distribution of the noise part of the matrix. This will cause it to behave more like a Wigner matrix, in that the spectral norm of the noise will not increase after a partial trace. Consider the partial trace of a matrix of the form

$$
M-\alpha \cdot \operatorname{Id} \otimes \sum_{i} a_{i} a_{i}^{\top}
$$

for some constant $\alpha>0$. The partial trace of this matrix is

$$
N^{\prime}=\sum_{i=1}^{n}\left(\left\|a_{i}\right\|_{2}^{2}-\alpha\right) \cdot a_{i} a_{i}^{\top} .
$$

We choose the constant $\alpha \approx d / n$ such that our matrix $N^{\prime}$ has expectation 0 in the No case, when the subspace is completely random. In the yes case, the Rayleigh quotient of $N^{\prime}$ at $x_{0}$ simply shifts as compared to $N$, and we have $\lambda_{\mathrm{YES}} \geqslant\left\langle x_{0}, N^{\prime} x_{0}\right\rangle \approx\left\|v_{0}\right\|_{4}^{4} \geqslant 1 / k$ (see Lemma 4.5 and sublemmas). On the other hand, in the No case, this centering operation causes significant cancellations in the 
eigenvalues of the partial trace matrix (instead of just shifting the eigenvalues). In the No case, $N^{\prime}$ has spectral norm $\lambda_{\mathrm{NO}} \leqslant O\left(d / n^{3 / 2}\right.$ ) for $d \ll \sqrt{n}$ (using standard matrix concentration bounds; again see Lemma 4.5 and sublemmas). Therefore, the spectral norm of the matrix $N^{\prime}$ allows us to distinguish between the YEs and No case as long as $d / n^{3 / 2} \ll 1 / k$, which is satisfied as long as $k \ll n$ and $d \ll \sqrt{n}$. We give the full formal argument in Section 4 .

\subsection{Overcomplete tensor decomposition}

Recall that in this problem we are given a 3-tensor $T$ of the form $T=\sum_{i=1}^{n} a_{i}^{\otimes 3} \in \mathbb{R}^{d^{3}}$, where $a_{1}, \ldots, a_{n} \in \mathbb{R}^{d}$ are independent random vectors from $N\left(0, \frac{1}{d} \mathrm{Id}\right)$. The goal is to find a unit vector $a \in \mathbb{R}^{d}$ that is highly correlated with one $\mathrm{e}^{4}$ of the vectors $a_{1}, \ldots, a_{n}$.

Background. The starting point of our algorithm is the polynomial $f(x)=\sum_{i=1}^{n}\left\langle a_{i}, x\right\rangle^{3}$. It turns out that for $n \ll d^{1.5}$ the (approximate) maximizers of this polynomial are close to the components $a_{1}, \ldots, a_{n}$, in the sense that $f(x) \approx 1$ if and only if $\max _{i \in[n]}\left\langle a_{i}, x\right\rangle^{2} \approx 1$. Indeed, Ge and Ma [GM15] show that the sum-of-squares method already captures this fact at degree 12, which implies a quasipolynomial time algorithm for this tensor decomposition problem via a general rounding result of Barak, Kelner, and Steurer [BKS15].

The simplest approach to this problem is to consider the tensor representation of the polynomial $\mathbf{T}=\sum_{i \in[n]} a_{i}^{\otimes 3}$, and flatten it, hoping the singular vectors of the flattening are correlated with the $a_{i}$. However, this approach is doomed to failure for two reasons: firstly, the simple flattenings of T are $d^{2} \times d$ matrices, and since $n \gg d$ the $a_{i}^{\otimes 2}$ collide in the column space, so that it is impossible to determine $\operatorname{Span}\left\{a_{i}^{\otimes 2}\right\}$. Secondly, even for $n \leqslant d$, because the $a_{i}$ are random vectors, their norms concentrate very closely about 1 . This makes it difficult to distinguish any one particular $a_{i}$ even when the span is computable.

Improvements. We will try to circumvent both of these issues by going to higher dimensions. Suppose, for example, that we had access to $\sum_{i \in[n]} a_{i}^{\otimes 4} .{ }^{5}$ The eigenvectors of the flattenings of this matrix are all within $\operatorname{Span}_{i \in[n]}\left\{a_{i}^{\otimes 2}\right\}$, addressing our first issue, leaving us only with the trouble of extracting individual $a_{i}^{\otimes 2}$ from their span. If furthermore we had access to $\sum_{i \in[n]} a_{i}^{\otimes 6}$, we could perform a partial random projection $(\Phi \otimes \mathrm{Id} \otimes \mathrm{Id}) \sum_{i \in[n]} a_{i}^{\otimes 6}$ where $\Phi \in \mathbb{R}^{d \times d}$ is a matrix with independent Gaussian entires, and then taking a partial trace, we end up with

$$
\operatorname{Tr}_{\mathbb{R}^{d}}\left((\Phi \otimes \mathrm{Id} \otimes \mathrm{Id}) \sum_{i \in[n]} a_{i}^{\otimes 6}\right)=\sum_{i \in[n]}\left\langle\Phi, a_{i}^{\otimes 2}\right\rangle a_{i}^{\otimes 4} .
$$

With reasonable probability (for exposition's sake, say with probability $1 / n^{10}$ ), $\Phi$ is closer to some $a_{i}^{\otimes 2}$ than to all of the others so that $\left\langle\Phi, a_{i}^{\otimes 2}\right\rangle \geqslant 100\left\langle\Phi, a_{j}^{\otimes 2}\right\rangle$ for all $j \in[n]$, and then $a_{i}^{\otimes 2}$ is distinguishable from the other vectors in the span of our matrix, taking care of the second issue. As we show, a much smaller gap is sufficient to distinguish the top $a_{i}$ from the other $a_{j}$, and so the higherprobability event that $\Phi$ is only slightly closer to $a_{i}$ suffices (allowing us to recover all vectors at

\footnotetext{
${ }^{4}$ We can then approximately recover all the components $a_{1}, \ldots, a_{n}$ by running independent trials of our randomized algorithm repeatedly on the same input.

${ }^{5}$ As the problem is defined, we assume that we do not have access to this input, and in many machine learning applications this is a valid assumption, as gathering the data necessary to generate the 4th order input tensor requires a prohibitively large number of samples.
} 
an additional runtime cost of a factor of $\tilde{O}(n))$. This discussion ignores the presence of a single spurious large eigenvector, which we address in the technical sections.

Of course, we do not have access to the higher-order tensor $\sum_{i \in[n]} a_{i}^{\otimes 6}$. Instead, we can obtain a noisy version of this tensor. Our approach considers the following matrix representation of the polynomial $f^{2}$,

$$
M=\sum_{i, j} a_{i} a_{j}^{\top} \otimes\left(a_{i} a_{i}^{\top}\right) \otimes\left(a_{j} a_{j}^{\top}\right) \in \mathbb{R}^{d^{3} \times d^{3}} .
$$

Alternatively, we can view this matrix as a particular flattening of the Kronecker-squared tensor $\mathbf{T}^{\otimes 2}$. It is instructive to decompose $M=M_{\text {diag }}+M_{\text {cross }}$ into its diagonal terms $M_{\text {diag }}=\sum_{i}\left(a_{i} a_{i}^{\top}\right)^{\otimes 3}$ and its cross terms $M_{\text {cross }}=\sum_{i \neq j} a_{i} a_{j}^{\top} \otimes\left(a_{i} a_{i}^{\top}\right) \otimes\left(a_{j} a_{j}^{\top}\right)$. The algorithm described above is already successful for $M_{\text {diag; }}$ we need only control the eigenvalues of the partial trace of the "noise" component, $M_{\text {cross. }}$. The main technical work will be to show that $\left\|\operatorname{Tr}_{\mathbb{R}^{d}} M_{\text {diag }}\right\|$ is small. In fact, we will have to choose $\Phi$ from a somewhat different distribution-observing that $\operatorname{Tr}_{\mathbb{R}^{d}}(\Phi \otimes \mathrm{Id} \otimes \mathrm{Id})=$ $\sum_{i, j}\left\langle a_{i}, \Phi a_{j}\right\rangle \cdot\left(a_{i} \otimes a_{j}\right)\left(a_{i} \otimes a_{j}\right)^{\top}$, we will sample $\Phi$ so that $\left\langle a_{i}, \Phi a_{i}\right\rangle \gg\left\langle a_{i}, \Phi a_{j}\right\rangle$. We give a more detailed overview of this algorithm in the beginning of Section 5, explaining in more detail our choice of $\Phi$ and justifying heuristically the boundedness of the spectral norm of the noise.

Connection to SoS analysis. To explain how the above algorithm is a speedup of SoS, we give an overview of the SoS algorithm of [GM15, BKS15]. There, the degree- $t$ SoS SDP program is used to obtain an order- $t$ tensor $\chi_{t}$ (or a pseudodistribution). Informally speaking, we can understand $\chi_{t}$ as a proxy for $\sum_{i \in[n]} a_{i}^{\otimes t}$, so that $\chi_{t}=\sum_{i \in[n]} a_{i}^{\otimes t}+N$, where $N$ is a noise tensor. While the precise form of $N$ is unclear, we know that $N$ must obey a set of constraints imposed by the SoS hierarchy at degree $t$. For a formal discussion of pseudodistributions, see [BKS15].

To extract a single component $a_{i}$ from the tensor $\sum_{i \in[n]} a_{i}^{\otimes t}$, there are many algorithms which would work (for example, the algorithm we described for $M_{\text {diag }}$ above). However, any algorithm extracting an $a_{i}$ from $\chi_{t}$ must be robust to the noise tensor $N$. For this it turns out the following algorithm will do: suppose we have the tensor $\sum_{i \in[n]} a_{i}^{\otimes t}$, taking $t=O(\log n)$. Sample $g_{1}, \ldots, g_{\log (n)-2}$ random unit vectors, and compute the matrix $M=\sum_{i}\left(\prod_{1 \leqslant j \leqslant \log (n)-2}\left\langle g_{j}, a_{i}\right\rangle\right) \cdot a_{i} a_{i}^{\top}$. If we are lucky enough, there is some $a_{i}$ so that every $g_{j}$ is a bit closer to $a_{i}$ than any other $a_{i^{\prime}}$, and $M=a_{i} a_{i}^{\top}+E$ for some $\|E\| \ll 1$. The proof that $\|E\|$ is small can be made so simple that it applies also to the SDP-produced proxy tensor $\chi_{\log n}$, and so this algorithm is robust to the noise $N$. This last step is very general and can handle tensors whose components $a_{i}$ are less well-behaved than the random vectors we consider, and also more overcomplete, handling tensors of rank up to $n=\tilde{\Omega}\left(d^{1.5}\right){ }^{6}$

Our subquadratic-time algorithm can be viewed as a low-degree, spectral analogue of the [BKS15] SoS algorithm. However, rather than relying on an SDP to produce an object close to $\sum_{i \in[n]} a_{i}^{\otimes t}$, we manufacture one ourselves by taking the Kronecker square of our input tensor. We explicitly know the form of the deviation of $\mathbf{T}^{\otimes 2}$ from $\sum_{i \in[n]} a_{i}^{\otimes 6}$, unlike in [BKS15], where the deviation of the SDP certificate $\chi_{t}$ from $\sum_{i \in[n]} a_{i}^{\otimes t}$ is poorly understood. We are thus able to control this deviation (or "noise") in a less computationally intensive way, by cleverly designing a partial trace operation which decreases the spectral norm of the deviation. Since the tensor handled by the algorithm is much smaller-order 6 rather than order $\log n$-this provides the desired speedup.

\footnotetext{
${ }^{6}$ It is an interesting open question whether taking $t=O(\log n)$ is really necessary, or whether this heavy computational requirement is simply an artifact of the SoS proof.
} 


\subsection{Tensor principal component analysis}

Recall that in this problem we are given a tensor $\mathbf{T}=\tau \cdot v^{\otimes 3}+\mathbf{A}$, where $v \in \mathbb{R}^{d}$ is a unit vector, $\mathbf{A}$ has iid entries from $\mathcal{N}(0,1)$, and $\tau>0$ is the signal-to-noise ratio. The aim is to recover $v$ approximately.

Background and SoS analysis. A previous application of SoS techniques to this problem discussed several SoS or spectral algorithms, including one that runs in quasi-linear time [HSS15]. Here we apply the partial trace method to a subquadratic spectral SoS algorithm discussed in [HSS15] to achieve nearly the same signal-to-noise guarantee in only linear time.

Our starting point is the polynomial $\mathbf{T}(x)=\tau \cdot\langle v, x\rangle^{3}+\left\langle x^{\otimes 3}, \mathbf{A}\right\rangle$. The maximizer of $\mathbf{T}(x)$ over the sphere is close to the vector $v$ so long as $\tau \gg \sqrt{n}$ [RM14]. In [HSS15], it was shown that degree- 4 SoS maximizing this polynomial can recover $v$ with a signal-to-noise ratio of at least $\tilde{\Omega}\left(n^{3 / 4}\right)$, since there exists a suitable SoS bound on the noise term $\left\langle x^{\otimes 3}, \mathbf{A}\right\rangle$.

Specifically, let $A_{i}$ be the $i$ th slice of $\mathbf{A}$, so that $\left\langle x, A_{i} x\right\rangle$ is the quadratic form $\sum_{j, k} \mathbf{A}_{i j k} x_{j} x_{k}$. Then there is a SoS proof that $\mathbf{T}(x)$ is bounded by $\left|\mathbf{T}(x)-\tau \cdot\langle v, x\rangle^{3}\right| \leqslant f(x)^{1 / 2} \cdot\|x\|$, where $f(x)$ is the degree-4 polynomial $f(x)=\sum_{i}\left\langle x, A_{i} x\right\rangle^{2}$. The polynomial $f$ has a convenient matrix representation: $f(x)=\left\langle x^{\otimes 2},\left(\sum_{i} A_{i} \otimes A_{i}\right) x^{\otimes 2}\right\rangle$ : since this matrix is a sum of iid random matrices $A_{i} \otimes A_{i}$, it is easy to show that this matrix spectrally concentrates to its expectation. So with high probability one can show that the eigenvalues of $\sum_{i} A_{i} \otimes A_{i}$ are at most $\approx d^{3 / 2} \log (d)^{1 / 2}$ (except for a single spurious eigenvector), and it follows that degree-4 SoS solves tensor PCA so long as $\tau \gg d^{3 / 4} \log (d)^{1 / 4}$.

This leads the authors to consider a slight modification of $f(x)$, given by $g(x)=\sum_{i}\left\langle x, T_{i} x\right\rangle^{2}$, where $T_{i}$ is the $i$ th slice of $\mathbf{T}$. Like $\mathbf{T}$, the function $g$ also contains information about $v$, and the SoS bound on the noise term in $\mathbf{T}$ carries over as an analogous bound on the noise in $g$. In particular, expanding $T_{i} \otimes T_{i}$ and ignoring some negligible cross-terms yields

$$
\sum_{i} T_{i} \otimes T_{i} \approx \tau^{2} \cdot(v \otimes v)(v \otimes v)^{\top}+\sum_{i} A_{i} \otimes A_{i}
$$

Using $v \otimes v$ as a test vector, the quadratic form of the latter matrix can be made at least $\tau^{2}-$ $O\left(d^{3 / 2} \log (d)^{1 / 2}\right)$. Together with the boundedness of the eigenvalues of $\sum_{i} A_{i} \otimes A_{i}$ this shows that when $\tau \gg d^{3 / 4} \log (d)^{1 / 4}$ there is a spectral algorithm to recover $v$. Since the matrix $\sum_{i} T_{i} \otimes T_{i}$ is $d^{2} \times d^{2}$, computing the top eigenvector requires $\tilde{O}\left(d^{4} \log n\right)$ time, and by comparison to the input size $d^{3}$ the algorithm runs in subquadratic time.

Improvements. In this work we speed this up to a linear time algorithm via the partial trace approach. As we have seen, the heart of the matter is to show that taking the partial trace of $\tau^{2} \cdot(v \otimes v)(v \otimes v)^{\top}+\sum_{i} A_{i} \otimes A_{i}$ does not increase the spectral noise. That is, we require that

$$
\left\|\operatorname{Tr}_{\mathbb{R}^{d}} \sum_{i} A_{i} \otimes A_{i}\right\|=\left\|\sum_{i} \operatorname{Tr}\left(A_{i}\right) \cdot A_{i}\right\| \leqslant O\left(d^{3 / 2} \log (d)^{1 / 2}\right) .
$$

Notice that the $A_{i}$ are essentially Wigner matrices, and so it is roughly true that $\left|\operatorname{Tr}\left(A_{i}\right)\right| \approx\left\|A_{i}\right\|$, and the situation is very similar to our toy example of the application of partial traces in Section 2.

Heuristically, because $\sum_{i \in[n]} A_{i} \otimes A_{i}$ and $\sum_{i \in[n]} \operatorname{Tr}\left(A_{i}\right) \cdot A_{i}$ are random matrices, we expect that their eigenvalues are all of roughly the same magnitude. This means that their spectral norm should be close to their Frobenius norm divided by the square root of the dimension, since for a matrix $M$ with eigenvalues $\lambda_{1}, \ldots, \lambda_{n},\|M\|_{F}=\sqrt{\sum_{i \in[n]} \lambda_{i}^{2}}$. By estimating the sum of the squared 
entries, we expect that the Frobenious norm of $\sum_{i} \operatorname{Tr}\left(A_{i}\right) \cdot A_{i}$ is less than that of $\sum_{i} A_{i} \otimes A_{i}$ by a factor of $\sqrt{d}$ after the partial trace, while the dimension decreases by a factor of $d$, and so assuming that the eigenvalues are all of the same order, a typical eigenvalue should remain unchanged. We formalize these heuristic calculations using standard matrix concentration arguments in Section 6.

\section{Preliminaries}

Linear algebra. We will work in the real vector spaces given by $\mathbb{R}^{n}$. A vector of indeterminates may be denoted $x=\left(x_{1}, \ldots, x_{n}\right)$, although we may sometimes switch to parenthetical notation for indexing, i.e. $x=(x(1), \ldots, x(n))$ when subscripts are already in use. We denote by $[n]$ the set of all valid indices for a vector in $\mathbb{R}^{n}$. Let $e_{i}$ be the $i$ th canonical basis vector so that $e_{i}(i)=1$ and $e_{i}(j)=0$ for $j \neq i$.

For a vectors space $V$, we may denote by $\mathcal{L}(V)$ the space of linear operators from $V$ to $V$. The space orthogonal to a vector $v$ is denoted $v^{\perp}$.

For a matrix $M$, we use $M^{-1}$ to denote its inverse or its Moore-Penrose pseudoinverse; which one it is will be clear from context. For $M$ PSD, we write $M^{-1 / 2}$ for the unique PSD matrix with $\left(M^{-1 / 2}\right)^{2}=M^{-1}$.

Norms and inner products. We denote the usual entrywise inner product by $\langle\cdot, \cdot\rangle$, so that $\langle u, v\rangle=$ $\sum_{i \in[n]} u_{i} v_{i}$ for $u, v \in \mathbb{R}^{n}$. The $\ell_{p}$-norm of a vector $v \in \mathbb{R}^{n}$ is given by $\|v\|_{p}=\left(\sum_{i \in[n]} v_{i}{ }^{p}\right)^{1 / p}$, with $\|v\|$ denoting the $\ell_{2}$-norm by default. The matrix norm used throughout the paper will be the operator / spectral norm, denoted by $\|M\|=\|M\|_{o p}:=\max _{x \neq 0}\|M x\| /\|x\|$.

Tensor manipulation. Boldface variables will reserved for tensors $\mathbf{T} \in \mathbb{R}^{n \times n \times n}$, of which we consider only order-3 tensors. We denote by $\mathbf{T}(x, y, z)$ the multilinear function in $x, y, z \in \mathbb{R}^{n}$ such that $\mathbf{T}(x, y, z)=\sum_{i, j, k \in[n]} T_{i, j, k} x_{i} y_{j} z_{k}$, applying $x, y$, and $z$ to the first, second, and third modes of the tensor $\mathbf{T}$ respectively. If the arguments are matrices $P, Q$, and $R$ instead, this lifts $\mathbf{T}(P, Q, R)$ to the unique multilinear tensor-valued function such that $[\mathbf{T}(P, Q, R)](x, y, z)=\mathbf{T}(P x, Q y, R z)$ for all vectors $x, y, z$.

Tensors may be flattened to matrices in the multilinear way such that for every $u \in \mathbb{R}^{n \times n}$ and $v \in \mathbb{R}^{n}$, the tensor $u \otimes v$ flattens to the matrix $u v^{\top} \in \mathbb{R}^{n^{2} \times n}$ with $u$ considered as a vector. There are 3 different ways to flatten a 3-tensor $\mathbf{T}$, corresponding to the 3 modes of $\mathbf{T}$. Flattening may be understood as reinterpreting the indices of a tensor when the tensor is expressed as an 3-dimensional array of numbers. The expression $v^{\otimes 3}$ refers to $v \otimes v \otimes v$ for a vector $v$.

Probability and asymptotic bounds. We will often refer to collections of independent and identically distributed (or iid) random variables. The Gaussian distribution with mean $\mu$ and variance $\sigma^{2}$ is denoted $\mathcal{N}\left(\mu, \sigma^{2}\right)$. Sometimes we state that an event happens with overwhelming probability. This means that its probability is at least $1-n^{-\omega(1)}$. A function is $\widetilde{O}(g(n))$ if it is $O(g(n))$ up to polylogarithmic factors. 


\section{Planted sparse vector in random linear subspace}

In this section we give a nearly-linear-time algorithm to recover a sparse vector planted in a random subspace.

Problem 4.1. Let $v_{0} \in \mathbb{R}^{n}$ be a unit vector such that $\left\|v_{0}\right\|_{4}^{4} \geqslant \frac{1}{\varepsilon n}$. Let $v_{1}, \ldots, v_{d-1} \in \mathbb{R}^{n}$ be iid from $\mathcal{N}\left(0, \frac{1}{n} \operatorname{Id}_{n}\right)$. Let $w_{0}, \ldots, w_{d-1}$ be an orthogonal basis for $\operatorname{Span}\left\{v_{0}, \ldots, v_{d-1}\right\}$. Given: $w_{0}, \ldots, w_{d-1}$ Find: a vector $v$ such that $\left\langle v, v_{0}\right\rangle^{2} \geqslant 1-o(1)$.

\section{Sparse Vector Recovery in Nearly-Linear Time}

Algorithm 4.2. Input: $w_{0}, \ldots, w_{d-1}$ as in Problem 4.1. Goal: Find $v$ with $\left\langle\hat{v}, v_{0}\right\rangle^{2} \geqslant 1-o(1)$.

- Compute leverage scores $\left\|a_{1}\right\|^{2}, \ldots,\left\|a_{n}\right\|^{2}$, where $a_{i}$ is the $i$ th row of the $n \times d$ matrix $S:=$ $\left(\begin{array}{lll}w_{0} & \cdots & w_{d-1}\end{array}\right)$.

- Compute the top eigenvector $u$ of the matrix

$$
A \stackrel{\text { def }}{=} \sum_{i \in[n]}\left(\left\|a_{i}\right\|_{2}^{2}-\frac{d}{n}\right) \cdot a_{i} a_{i}^{\top}
$$

- Output Su.

Remark 4.3 (Implementation of Algorithm 4.2 in nearly-linear time). The leverage scores $\left\|a_{1}\right\|^{2}, \ldots,\left\|a_{n}\right\|^{2}$ are clearly computable in time $O(n d)$. In the course of proving correctness of the algorithm we will show that $A$ has constant spectral gap, so by a standard analysis $O(\log d)$ matrix-vector multiplies suffice to recover its top eigenvector. A single matrix-vector multiply $A x$ requires computing $c_{i}:=\left(\left\|a_{i}\right\|^{2}-\frac{d}{n}\right)\left\langle a_{i}, x\right\rangle$ for each $i$ (in time $O(n d)$ ) and summing $\sum_{i \in[n]} c_{i} x_{i}$ (in time $O(n d)$ ). Finally, computing $S u$ requires summing $d$ vectors of dimension $n$, again taking time $O(n d)$.

The following theorem expresses correctness of the algorithm.

Theorem 4.4. Let $v_{0} \in \mathbb{R}^{n}$ be a unit vector with $\left\|v_{0}\right\|_{4}^{4} \geqslant \frac{1}{\varepsilon n}$. Let $v_{1}, \ldots, v_{d-1} \in \mathbb{R}^{n}$ be iid from $\mathcal{N}\left(0, \frac{1}{n} \operatorname{Id}_{n}\right)$. Let $w_{0}, \ldots, w_{d-1}$ be an orthogonal basis for $\operatorname{Span}\left\{v_{0}, \ldots, v_{d-1}\right\}$. Let $a_{i}$ be the $i$-th row of the $n \times d$ matrix $S:=\left(\begin{array}{ccc}w_{0} & \cdots & w_{d-1}\end{array}\right)$.

When $d \leqslant n^{1 / 2} / \operatorname{polylog}(n)$, for any sparsity $\varepsilon>0$, w.ov.p. the top eigenvector $u$ of $\sum_{i=1}^{n}\left(\left\|a_{i}\right\|^{2}-\frac{d}{n}\right) \cdot a_{i} a_{i}^{\top}$ has $\left\langle S u, v_{0}\right\rangle^{2} \geqslant 1-O\left(\varepsilon^{1 / 4}\right)-o(1)$.

We have little control over the basis vectors the algorithm is given. However, there is a particularly nice (albeit non-orthogonal) basis for the subspace which exposes the underlying randomness. Suppose that we are given the basis vectors $v_{0}, \ldots, v_{d}$, where $v_{0}$ is the sparse vector normalized so that $\left\|v_{0}\right\|=1$, and $v_{1}, \ldots, v_{d-1}$ are iid samples from $\mathcal{N}\left(0, \frac{1}{n} \operatorname{Id}_{n}\right)$. The following lemma shows that if the algorithm had been handed this good representation of the basis rather than an arbitrary orthogonal one, its output would be the correlated to the vector of coefficients giving of the planted sparse vector (in this case the standard basis vector $e_{1}$ ). 
Lemma 4.5. Let $v_{0} \in \mathbb{R}^{n}$ be a unit vector. Let $v_{1}, \ldots, v_{d-1} \in \mathbb{R}^{n}$ be iid from $\mathcal{N}\left(0, \frac{1}{n} \mathrm{Id}\right)$. Let $a_{i}$ be the ith row of the $n \times d$ matrix $S:=\left(\begin{array}{lll}v_{0} & \cdots & v_{d-1}\end{array}\right)$. Then there is a universal constant $\varepsilon^{*}>0$ so that for any $\varepsilon \leqslant \varepsilon^{*}$, so long as $d \leqslant n^{1 / 2} / \operatorname{polylog}(n)$, w.ov.p.

$$
\sum_{i=1}^{n}\left(\left\|a_{i}\right\|^{2}-\frac{d}{n}\right) \cdot a_{i} a_{i}^{\top}=\left\|v_{0}\right\|_{4}^{4} \cdot e_{1} e_{1}^{\top}+M
$$

where $e_{1}$ is the first standard basis vector and $\|M\| \leqslant O\left(\left\|v_{0}\right\|_{4}^{3} \cdot n^{-1 / 4}+\left\|v_{0}\right\|_{4}^{2} \cdot n^{-1 / 2}+\left\|v_{0}\right\|_{4} \cdot n^{-3 / 4}+n^{-1}\right)$.

The second ingredient we need is that the algorithm is robust to exchanging this good basis for an arbitrary orthogonal basis.

Lemma 4.6. Let $v_{0} \in \mathbb{R}^{n}$ have $\left\|v_{0}\right\|_{4}^{4} \geqslant \frac{1}{\varepsilon n}$. Let $v_{1}, \ldots, v_{d-1} \in \mathbb{R}^{n}$ be iid from $\mathcal{N}\left(0, \frac{1}{n} \operatorname{Id}_{n}\right)$. Let $w_{0}, \ldots, w_{d-1}$ be an orthogonal basis for $\operatorname{Span}\left\{v_{0}, \ldots, v_{d-1}\right\}$. Let $a_{i}$ be the ith row of the $n \times d$ matrix $S:=\left(\begin{array}{lll}v_{0} & \cdots & v_{d-1}\end{array}\right)$. Let $a_{i}^{\prime}$ be the ith row of the $n \times d$ matrix $S^{\prime}:=\left(\begin{array}{lll}w_{0} & \cdots & w_{d-1}\end{array}\right)$. Let $A:=\sum_{i} a_{i} a_{i}^{\top}$. Let $Q \in \mathbb{R}^{d \times d}$ be the orthogonal matrix so that $S A^{-1 / 2}=S^{\prime} Q$, which exists since $S A^{-1 / 2}$ is orthogonal, and which has the effect that $a_{i}^{\prime}=Q A^{-1 / 2} a_{i}$. Then when $d \leqslant n^{1 / 2} / \operatorname{polylog}(n)$, w.ov.p.

$$
\left\|\sum_{i=1}^{n}\left(\left\|a_{i}^{\prime}\right\|^{2}-\frac{d}{n}\right) \cdot a_{i}^{\prime} a_{i}^{\prime \top}-Q\left(\sum_{i=1}^{n}\left(\left\|a_{i}\right\|^{2}-\frac{d}{n}\right) \cdot a_{i} a_{i}^{\top}\right) Q^{\top}\right\| \leqslant O\left(\frac{1}{n}\right)+o\left(\|v\|_{4}^{4}\right)
$$

Last, we will need the following fact, which follows from standard concentration. The proof is in Section B.

Lemma 4.7. Let $v \in \mathbb{R}^{n}$ be a unit vector. Let $b_{1}, \ldots, b_{n} \in \mathbb{R}^{d-1}$ be iid from $\mathcal{N}\left(0, \frac{1}{n} \operatorname{Id}_{d-1}\right)$. Let $a_{i} \in \mathbb{R}^{d}$ be given by $a_{i}:=\left(v(i) b_{i}\right)$. Then w.ov.p. $\left\|\sum_{i=1}^{n} a_{i} a_{i}^{\top}-\operatorname{Id}_{d}\right\| \leqslant \tilde{O}(d / n)^{1 / 2}$. In particular, when $d=o(n)$, this implies that w.ov.p. $\left\|\left(\sum_{i=1}^{n} a_{i} a_{i}^{\top}\right)^{-1}-\operatorname{Id}_{d}\right\| \leqslant \tilde{O}(d / n)^{1 / 2}$ and $\left\|\left(\sum_{i=1}^{n} a_{i} a_{i}^{\top}\right)^{-1 / 2}-\operatorname{Id}_{d}\right\| \leqslant \tilde{O}(d / n)^{1 / 2}$.

We are ready to prove Theorem 4.4 .

Proof of Theorem 4.4. Let $b_{1}, \ldots, b_{n}$ be the rows of the matrix $S^{\prime}:=\left(\begin{array}{ccc}v_{0} & \cdots & v_{d-1}\end{array}\right)$. Let $B=\sum_{i} b_{i} b_{i}^{\top}$. Note that $S^{\prime} B^{-1 / 2}$ has columns which are an orthogonal basis for $\operatorname{Span}\left\{w_{0}, \ldots, w_{d-1}\right\}$. Let $Q \in \mathbb{R}^{d \times d}$ be the rotation so that $S^{\prime} B^{-1 / 2}=S Q$.

By Lemma 4.5 and Lemma 4.6, we can write the matrix $A=\sum_{i=1}^{n}\left(\left\|a_{i}\right\|_{2}^{2}-\frac{d}{n}\right) \cdot a_{i} a_{i}^{\top}$ as

$$
A=\left\|v_{0}\right\|_{4}^{4} \cdot Q e_{1} e_{1}^{\top} Q^{\top}+M
$$

where w.ov.p.

$$
\|M\| \leqslant O\left(\left\|v_{0}\right\|_{4}^{3} \cdot n^{-1 / 4}+\left\|v_{0}\right\|_{4}^{2} \cdot n^{-1 / 2}+\left\|v_{0}\right\|_{4} \cdot n^{-3 / 4}+n^{-1}\right)+o\left(\|v\|_{4}^{4}\right) .
$$

We have assumed that $\left\|v_{0}\right\|_{4}^{4} \geqslant(\varepsilon n)^{-1}$, and so since $A$ is an almost-rank-one matrix (Lemma A.3), the top eigenvector $u$ of $A$ has $\left\langle u, Q e_{1}\right\rangle^{2} \geqslant 1-O\left(\varepsilon^{1 / 4}\right)$, so that $\left\langle S u, S Q e_{1}\right\rangle^{2} \geqslant 1-O\left(\varepsilon^{1 / 4}\right)$ by columnorthogonality of $S$.

At the same time, $S Q e_{1}=S^{\prime} B^{-1 / 2} e_{1}$, and by Lemma $4.7,\left\|B^{-1 / 2}-\operatorname{Id}\right\| \leqslant \tilde{O}(d / n)^{1 / 2}$ w.ov.p., so that $\left\langle S u, S^{\prime} e_{1}\right\rangle^{2} \geqslant\left\langle S u, S Q e_{1}\right\rangle^{2}-o(1)$. Finally, $S^{\prime} e_{1}=v_{0}$ by definition, so $\left\langle S u, v_{0}\right\rangle^{2} \geqslant 1-O\left(\varepsilon^{1 / 4}\right)-o(1)$. 


\subsection{Algorithm succeeds on good basis}

We now prove Lemma 4.5. We decompose the matrix in question into a contribution from $\left\|v_{0}\right\|_{4}^{4}$ and the rest: explicitly, the decomposition is $\sum\left(\left\|a_{i}\right\|_{2}^{2}-\frac{d}{n}\right) \cdot a_{i} a_{i}^{\top}=\sum v(i)^{2} \cdot a_{i} a_{i}^{\top}+\sum\left(\left\|b_{i}\right\|_{2}^{2}-\frac{d}{n} \cdot a_{i} a_{i}^{\top}\right)$. This first lemma handles the contribution from $\left\|v_{0}\right\|_{4}^{4}$.

Lemma 4.8. Let $v \in \mathbb{R}^{n}$ be a unit vector. Let $b_{1}, \ldots, b_{n} \in \mathbb{R}^{d-1}$ be random vectors iid from $\mathcal{N}\left(0, \frac{1}{n} \cdot \operatorname{Id}_{d-1}\right)$. Let $a_{i}=\left(v(i) b_{i}\right) \in \mathbb{R}^{d}$. Suppose $d \leqslant n^{1 / 2} / \operatorname{polylog}(n)$. Then

$$
\sum_{i=1}^{n} v(i)^{2} \cdot a_{i} a_{i}^{\top}=\|v\|_{4}^{4} \cdot e_{1} e_{1}^{\top}+M^{\prime}
$$

where $\left\|M^{\prime}\right\| \leqslant O\left(\|v\|_{4}^{3} n^{-1 / 4}+\|v\|_{4}^{2} n^{-1 / 2}\right)$ w.ov.p..

Proof of Lemma 4.8. We first show an operator-norm bound on the principal submatrix $\sum_{i=1}^{n} v(i)^{2}$. $b_{i} b_{i}^{\top}$ using the truncated matrix Bernstein inequality Proposition A.7. First, the expected operator norm of each summand is bounded:

$$
\mathbb{E} v(i)^{2}\left\|b_{i}\right\|_{2}^{2} \leqslant\left(\max _{j} v(j)^{2}\right) \cdot O\left(\frac{d}{n}\right) \leqslant\|v\|_{4}^{2} \cdot O\left(\frac{d}{n}\right) .
$$

The operator norms are bounded by constant-degree polynomials in Gaussian variables, so Lemma A.8 applies to truncate their tails in preparation for application of a Bernstein bound. We just have to calculate the variance of the sum, which is at most

$$
\left\|\mathbb{E} \sum_{i=1}^{n} v(i)^{4}\right\| b_{i}\left\|_{2}^{2} \cdot b_{i} b_{i}^{\top}\right\|=\|v\|_{4}^{4} \cdot O\left(\frac{d}{n^{2}}\right) .
$$

The expectation $\mathbb{E} \sum_{i=1}^{n} v(i)^{2} \cdot b_{i} b_{i}^{\top}$ is $\frac{\|v\|^{2}}{n} \cdot \mathrm{Id}$. Applying a matrix Bernstein bound (Proposition A.7) to the deviation from expectation, we get that w.ov.p.,

$$
\left\|\left(\sum_{i=1}^{n} v(i)^{2} \cdot b_{i} b_{i}^{\top}\right)-\frac{1}{n} \cdot \operatorname{Id}\right\| \leqslant\|v\|_{4}^{2} \cdot \tilde{O}\left(\frac{d}{n}\right) \leqslant O\left(\|v\|_{4}^{2} n^{-1 / 2}\right)
$$

for appropriate choice of $d \leqslant n^{-1 / 2} / \operatorname{polylog}(n)$. Hence, by triangle inequality, $\left\|\sum_{i=1}^{n} v(i)^{2} \cdot b_{i} b_{i}^{\top}\right\| \leqslant$ $\|v\|_{4}^{2} n^{-1 / 2}$ w.ov.p..

Using a Cauchy-Schwarz-style inequality (Lemma A.1) we now show that the bound on this principal submatrix is essentially enough to obtain the lemma. Let $p_{i}, q_{i} \in \mathbb{R}^{d}$ be given by

$$
p_{i} \stackrel{\text { def }}{=} v_{0}(i) \cdot\left(\begin{array}{c}
v_{0}(i) \\
0 \\
\vdots \\
0
\end{array}\right) \quad q_{i} \stackrel{\text { def }}{=} v_{0}(i) \cdot\left(\begin{array}{c}
0 \\
b_{i}
\end{array}\right) .
$$

Then

$$
\sum_{i=1}^{n} v(i)^{2} \cdot b_{i} b_{i}^{\top}=\|v\|_{4}^{4}+\sum_{i=1}^{n} p_{i} q_{i}^{\top}+q_{i} p_{i}^{\top}+q_{i} q_{i}^{\top}
$$


We have already bounded $\sum_{i=1}^{n} q_{i} q_{i}^{\top}=\sum_{i=1}^{n} v(i)^{2} \cdot b_{i} b_{i}^{\top}$. At the same time, $\left\|\sum_{i=1}^{n} p_{i} p_{i}^{\top}\right\|=\|v\|_{4}^{4}$. By Lemma A.1, then,

$$
\left\|\sum_{i=1}^{n} p_{i} q_{i}^{\top}+q_{i} p_{i}^{\top}\right\| \leqslant O\left(\|v\|_{4}^{3} n^{-1 / 4}\right)
$$

w.ov.p.. A final application of triangle inquality gives the lemma.

Our second lemma controls the contribution from the random part of the leverage scores.

Lemma 4.9. Let $v \in \mathbb{R}^{n}$ be a unit vector. Let $b_{1}, \ldots, b_{n} \in \mathbb{R}^{d-1}$ be random vectors iid from $\mathcal{N}\left(0, \frac{1}{n} \cdot \operatorname{Id}_{d-1}\right)$. Let $a_{i}=\left(v(i) b_{i}\right) \in \mathbb{R}^{d}$. Suppose $d \leqslant n^{1 / 2} / \operatorname{polylog}(n)$. Then w.ov.p.

$$
\left\|\sum_{i=1}^{n}\left(\left\|b_{i}\right\|_{2}^{2}-\frac{d}{n}\right) \cdot a_{i} a_{i}^{\top}\right\| \leqslant\|v\|_{4}^{2} \cdot O\left(n^{-3 / 4}\right)+\|v\|_{4} \cdot O\left(n^{-1}\right)+O\left(n^{-1}\right) .
$$

Proof. Like in the proof of Lemma 4.8, $\sum_{i=1}^{n}\left(\left\|b_{i}\right\|_{2}^{2}-\frac{d}{n}\right) \cdot a_{i} a_{i}^{\top}$ decomposes into a convenient block structure; we will bound each block separately.

$$
\sum_{i=1}^{n}\left(\left\|b_{i}\right\|_{2}^{2}-\frac{d}{n}\right) \cdot a_{i} a_{i}^{\top}=\sum_{i=1}^{n}\left(\left\|b_{i}\right\|_{2}^{2}-\frac{d}{n}\right) \cdot\left(\begin{array}{cc}
v(i)^{2} & v(i) \cdot b_{i}^{\top} \\
v(i) \cdot b_{i} & b_{i} b_{i}^{\top}
\end{array}\right) .
$$

In each block we can apply a (truncated) Bernstein inequality. For the large block $\sum_{i=1}^{n}\left(\left\|b_{i}\right\|_{2}^{2}-\frac{d}{n}\right) b_{i} b_{i}^{\top}$, the choice $\frac{d}{n}$ ensures that $\mathbb{E}\left(\left\|b_{i}\right\|_{2}^{2}-\frac{d}{n}\right) b_{i} b_{i}^{\top}=O\left(\frac{1}{n^{2}}\right) \cdot \mathrm{Id}$. The expected operator norm of each summand is small:

$$
\begin{aligned}
\mathbb{E}\left\|\left(\left\|b_{i}\right\|_{2}^{2}-\frac{d}{n}\right) b_{i} b_{i}^{\top}\right\| & =\mathbb{E} \mid\left(\left\|b_{i}\right\|_{2}^{2}-\frac{d}{n}\right)\left\|b_{i}\right\|_{2}^{2} \\
& \leqslant\left(\mathbb{E}\left(\left\|b_{i}\right\|_{2}^{2}-\frac{d}{n}\right)^{2}\right)^{1 / 2}\left(\mathbb{E}\left\|b_{i}\right\|_{2}^{4}\right)^{1 / 2} \quad \text { by Cauchy-Schwarz } \\
& \leqslant O\left(\frac{d^{1 / 2}}{n}\right) \cdot O\left(\frac{d}{n}\right) \quad \text { variance of } \chi^{2} \text { with } k \text { degrees of freedom is } O(k) \\
& =O\left(\frac{d^{3 / 2}}{n^{2}}\right) .
\end{aligned}
$$

The termwise operator norms are bounded by constant-degree polynomials in Gaussian variables, so Lemma A.8 applies to truncate the tails of the summands in preparation for a Bernstein bound. We just have to compute the variance of the sum, which is small because we have centered the coefficients:

$$
\left\|\sum_{i} \mathbb{E}\left(\left\|b_{i}\right\|_{2}^{2}-\frac{d}{n}\right)^{2}\right\| b_{i}\left\|_{2}^{2} \cdot b_{i} b_{i}^{\top}\right\| \leqslant O\left(\frac{d^{2}}{n^{3}}\right)
$$

by direct computation of $\mathbb{E}\left(\left\|b_{i}\right\|_{2}^{2}-\frac{d}{n}\right)^{2}\left\|b_{i}\right\|_{2}^{2} b_{i} b_{i}^{\top}$ using Fact A.6. These facts together are enough to apply the matrix Bernstein inequality (Proposition A.7) and conclude that w.ov.p.

$$
\left\|\sum_{i=1}^{n}\left(\left\|b_{i}\right\|_{2}^{2}-\frac{d}{n}\right) \cdot b_{i} b_{i}^{\top}\right\| \leqslant \tilde{O}\left(\frac{d}{n^{3 / 2}}\right) \leqslant O\left(\frac{1}{n}\right)
$$

for appropriate choice of $d \leqslant n / \operatorname{polylog}(n)$. 
We turn to the other blocks from (4.1). The upper-left block contains just the scalar $\sum_{i=1}^{n}\left(\left\|b_{i}\right\|_{2}^{2}-\right.$ $\left.\frac{d}{n}\right) v(i)^{2}$. By standard concentration each term is bounded: w.ov.p.,

$$
\left(\left\|b_{i}\right\|_{2}^{2}-\frac{d}{n}\right) v(i)^{2} \leqslant\left(\max _{i} v(i)^{2}\right) \cdot \tilde{O}\left(\frac{d^{1 / 2}}{n}\right) \leqslant\|v\|_{4}^{2} \cdot \tilde{O}\left(\frac{d^{1 / 2}}{n}\right) .
$$

The sum has variance at most $\sum_{i=1}^{n} v(i)^{4} \mathbb{E}\left(\left\|b_{i}\right\|_{2}^{2}-\frac{d}{n}\right)^{2} \leqslant\|v\|_{4}^{4} \cdot O\left(d / n^{2}\right)$. Again using Lemma A.8 and Proposition A.7, we get that w.ov.p.

$$
\left|\sum_{i=1}^{n}\left(\left\|b_{i}\right\|_{2}^{2}-\frac{d}{n}\right) v(i)^{2}\right| \leqslant\|v\|_{4}^{2} \cdot \tilde{O}\left(\frac{d^{1 / 2}}{n}\right) .
$$

It remains just to address the block $\sum_{i=1}^{n}\left(\left\|b_{i}\right\|_{2}^{2}-\frac{d}{n}\right) v(i) \cdot b_{i}$. Each term in the sum has expected operator norm at most

$$
\left(\max _{i} v(i)^{2}\right)^{1 / 2} \cdot O\left(\frac{d}{n^{3 / 2}}\right) \leqslant\|v\|_{4} \cdot O\left(\frac{d}{n^{3 / 2}}\right),
$$

and once again the since the summands' operator norms are bounded by constant-degree polynomials of Gaussian variables Lemma A.8 applies to truncate their tails in preparation to apply a Bernstein bound. The variance of the sum is at most $\|v\|_{2}^{2} \cdot O\left(d^{2} / n^{3}\right)$, again by Fact A.6. Finally, Lemma A.8 and Proposition A.7 apply to give that w.ov.p.

$$
\left\|\sum_{i=1}^{n}\left(\left\|b_{i}\right\|_{2}^{2}-\frac{d}{n}\right) v(i) \cdot b_{i}\right\| \leqslant\|v\|_{4} \cdot \tilde{O}\left(\frac{d}{n^{3 / 2}}\right)+\tilde{O}\left(\frac{d}{n^{3 / 2}}\right)=\|v\|_{4} \cdot n^{-1}+n^{-1}
$$

for appropriate choice of $d \leqslant n^{1 / 2} / \operatorname{polylog}(n)$. Putting it all together gives the lemma.

We are now ready to prove Lemma 4.5

Proof of Lemma 4.5. We decompose $\left\|a_{i}\right\|_{2}^{2}=v_{0}(i)^{2}+\left\|b_{i}\right\|_{2}^{2}$ and use Lemma 4.8 and Lemma 4.9.

$$
\begin{aligned}
\sum_{i=1}^{n}\left(\left\|a_{i}\right\|_{2}^{2}-\frac{d}{n}\right) \cdot a_{i} a_{i}^{\top} & =\left(\sum_{i=1}^{n} v_{0}(i)^{2} \cdot a_{i} a_{i}^{\top}\right)+\left(\sum_{i=1}^{n}\left(\left\|b_{i}\right\|_{2}^{2}-\frac{d}{n}\right) \cdot a_{i} a_{i}^{\top}\right) \\
& =\left\|v_{0}\right\|_{4}^{4} \cdot e_{1} e_{1}^{\top}+M,
\end{aligned}
$$

where

$$
\|M\| \leqslant O\left(\left\|v_{0}\right\|_{4}^{3} \cdot n^{-1 / 4}+\left\|v_{0}\right\|_{4}^{2} \cdot n^{-1 / 2}\right)+O\left(\left\|v_{0}\right\|_{4} \cdot n^{-1}+n^{-1}\right) .
$$

Since $\left\|v_{0}\right\|_{4}^{4} \geqslant(\varepsilon n)^{-1}$, we get $\left\|v_{0}\right\|_{4}^{4} /\|M\| \geqslant \frac{1}{\varepsilon^{1 / 4}}$, completing the proof.

\subsection{Closeness of input basis and good basis}

We turn now to the proof of Lemma 4.6. We recall the setting. We have two matrices: $M$, which the algorithm computes, and $M^{\prime}$, which is induced by a basis for the subspace which reveals the underlying randomness and which we prefer for the analysis. $M^{\prime}$ differs from $M$ by a rotation and a basis orthogonalization step (the good basis is only almost orthogonal). The rotation is easily handled. The following lemma gives the critical fact about the orthogonalization step: orthogonalizing does not change the leverage scores too much. ${ }^{7}$

\footnotetext{
${ }^{7}$ Strictly speaking the good basis does not have leverage scores since it is not orthogonal, but we can still talk about the norms of the rows of the matrix whose columns are the basis vectors.
} 
Lemma 4.10 (Restatement of Lemma B.4). Let $v \in \mathbb{R}^{n}$ be a unit vector and let $b_{1}, \ldots, b_{n} \in \mathbb{R}^{d-1}$ be iid from $\mathcal{N}\left(0, \frac{1}{n} \operatorname{Id}_{d-1}\right)$. Let $a_{i} \in \mathbb{R}^{d}$ be given by $a_{i}:=\left(v(i) b_{i}\right)$. Let $A:=\sum_{i} a_{i} a_{i}^{\top}$. Let $c \in \mathbb{R}^{d-1}$ be given by $c:=\sum_{i} v(i) b_{i}$. Then for every index $i \in[n]$, w.ov.p.,

$$
\left|\left\|A^{-1 / 2} a_{i}\right\|^{2}-\left\|a_{i}\right\|^{2}\right| \leqslant \tilde{O}\left(\frac{d+\sqrt{n}}{n}\right) \cdot\left\|a_{i}\right\|^{2} .
$$

The proof again uses standard concentration and matrix inversion formulas, and can be found in Section B. We are ready to prove Lemma 4.6.

Proof of Lemma 4.6. The statement we want to show is

$$
\left\|\sum_{i=1}^{n}\left(\left\|a_{i}^{\prime}\right\|^{2}-\frac{d}{n}\right) \cdot a_{i}^{\prime} a_{i}^{\prime \top}-Q\left(\sum_{i=1}^{n}\left(\left\|a_{i}\right\|^{2}-\frac{d}{n}\right) \cdot a_{i} a_{i}^{\top}\right) Q^{\top}\right\| \leqslant O\left(\frac{1}{n}\right)+o\left(\|v\|_{4}^{4}\right) .
$$

Conjugating by $Q$ and multiplying by -1 does not change the operator norm, so that this is equivalent to

$$
\left\|\sum_{i=1}^{n}\left(\left\|a_{i}\right\|^{2}-\frac{d}{n}\right) \cdot a_{i} a_{i}^{\top}-Q^{\top}\left(\sum_{i=1}^{n}\left(\left\|a_{i}^{\prime}\right\|^{2}-\frac{d}{n}\right) \cdot a_{i}^{\prime} a_{i}^{\top}\right) Q\right\| \leqslant O\left(\frac{1}{n}\right)+o\left(\|v\|_{4}^{4}\right) .
$$

Finally, substituting $a_{i}^{\prime}=Q A^{-1 / 2} a_{i}$, and using the fact that $Q$ is a rotation, it will be enough to show

$$
\left\|\left(\sum_{i=1}^{n}\left(\left\|a_{i}\right\|^{2}-\frac{d}{n}\right) \cdot a_{i} a_{i}^{\top}\right)-A^{-1 / 2}\left(\sum_{i=1}^{n}\left(\left\|A^{-1 / 2} a_{i}\right\|^{2}-\frac{d}{n}\right) \cdot a_{i} a_{i}^{\top}\right) A^{-1 / 2}\right\| \leqslant O\left(\frac{1}{n}\right)+o\left(\|v\|_{4}^{4}\right) .
$$

We write the right-hand matrix as

$$
\begin{aligned}
& A^{-1 / 2}\left(\sum_{i=1}^{n}\left(\left\|A^{-1 / 2} a_{i}\right\|^{2}-\frac{d}{n}\right) \cdot a_{i} a_{i}^{\top}\right) A^{-1 / 2} \\
& =A^{-1 / 2}\left(\sum_{i=1}^{n}\left(\left\|A^{-1 / 2} a_{i}\right\|^{2}-\left\|a_{i}\right\|^{2}\right) \cdot a_{i} a_{i}^{\top}\right) A^{-1 / 2}+A^{-1 / 2}\left(\sum_{i=1}^{n}\left(\left\|a_{i}\right\|^{2}-\frac{d}{n}\right) \cdot a_{i} a_{i}^{\top}\right) A^{-1 / 2} .
\end{aligned}
$$

The first of these we observe has bounded operator norm w.ov.p.:

$$
\begin{aligned}
\left\|A^{-1 / 2}\left(\sum_{i=1}^{n}\left(\left\|A^{-1 / 2} a_{i}\right\|^{2}-\left\|a_{i}\right\|^{2}\right) \cdot a_{i} a_{i}^{\top}\right) A^{-1 / 2}\right\| & \leqslant\left\|A^{-1 / 2}\left(\sum_{i=1}^{n}\left\|A^{-1 / 2} a_{i}\right\|^{2}-\left\|a_{i}\right\|^{2} \mid \cdot a_{i} a_{i}^{\top}\right) A^{-1 / 2}\right\| \\
& \leqslant \tilde{O}\left(\frac{d+\sqrt{n}}{n}\right) \cdot\left\|\sum_{i=1}^{n}\right\| a_{i}\left\|^{2} \cdot a_{i} a_{i}^{\top}\right\|
\end{aligned}
$$

where we have used Lemma 4.7 to find that $A^{1 / 2}$ is close to identity, and Lemma 4.10 to simplify the summands

$$
=\tilde{O}\left(\frac{d+\sqrt{n}}{n}\right) \cdot\left(\left\|\sum_{i=1}^{n} v_{0}(i)^{2} \cdot a_{i} a_{i}^{\top}\right\|+\left\|\sum_{i=1}^{n}\right\| b_{i}\left\|_{2}^{2} \cdot a_{i} a_{i}^{\top}\right\|\right)
$$




$$
\leqslant \tilde{O}\left(\frac{d+\sqrt{n}}{n}\right) \cdot\left(O\left(\|v\|_{4}^{4}\right)+\tilde{O}\left(\frac{d}{n}\right)\right),
$$

using in the last step Lemma 4.8 and standard concentration to bound $\sum_{i=1}^{n}\left\|b_{i}\right\|_{2}^{2} \cdot a_{i} a_{i}^{\top}$ (Lemma 4.7). Thus, by triangle inequality applied to (4.2), we get

$$
\begin{aligned}
& \left\|\left(\sum_{i=1}^{n}\left(\left\|a_{i}\right\|^{2}-\frac{d}{n}\right) \cdot a_{i} a_{i}^{\top}\right)-A^{-1 / 2}\left(\sum_{i=1}^{n}\left(\left\|A^{-1 / 2} a_{i}\right\|^{2}-\frac{d}{n}\right) \cdot a_{i} a_{i}^{\top}\right) A^{-1 / 2}\right\| \\
& \leqslant \tilde{O}\left(\frac{d+\sqrt{n}}{n}\right) \cdot\left(O\left(\|v\|_{4}^{4}\right)+\tilde{O}\left(\frac{d}{n}\right)\right)+\left\|\left(\sum_{i=1}^{n}\left(\left\|a_{i}\right\|^{2}-\frac{d}{n}\right) \cdot a_{i} a_{i}^{\top}\right)-A^{-1 / 2}\left(\sum_{i=1}^{n}\left(\left\|a_{i}\right\|^{2}-\frac{d}{n}\right) \cdot a_{i} a_{i}^{\top}\right) A^{-1 / 2}\right\| .
\end{aligned}
$$

Finally, since w.ov.p. $\left\|A^{-1 / 2}-\operatorname{Id}\right\|=\tilde{O}(d / n)^{1 / 2}$, we get

$$
\begin{aligned}
& \left\|\left(\sum_{i=1}^{n}\left(\left\|a_{i}\right\|^{2}-\frac{d}{n}\right) \cdot a_{i} a_{i}^{\top}\right)-A^{-1 / 2}\left(\sum_{i=1}^{n}\left(\left\|A^{-1 / 2} a_{i}\right\|^{2}-\frac{d}{n}\right) \cdot a_{i} a_{i}^{\top}\right) A^{-1 / 2}\right\| \\
& \leqslant \tilde{O}\left(\frac{d+\sqrt{n}}{n}\right) \cdot\left(O\left(\|v\|_{4}^{4}\right)+\tilde{O}\left(\frac{d}{n}\right)\right)+\tilde{O}\left(\frac{d}{n}\right)^{1 / 2} \cdot\left\|\sum_{i=1}^{n}\left(\left\|a_{i}\right\|_{2}^{2}-\frac{d}{n}\right) \cdot a_{i} a_{i}^{\top}\right\| \\
& \leqslant \tilde{O}\left(\frac{d+\sqrt{n}}{n}\right) \cdot\left(O\left(\|v\|_{4}^{4}\right)+\tilde{O}\left(\frac{d}{n}\right)\right)+\tilde{O}\left(\frac{d}{n}\right)^{1 / 2} \cdot O\left(\|v\|_{4}^{4}\right) .
\end{aligned}
$$

using Lemma 4.5 in the last step. For appropriate choice of $d \leqslant n^{-1 / 2} / \operatorname{polylog}(n)$, this is at most $O\left(n^{-1}\right)+o\left(\|v\|_{4}^{4}\right)$.

\section{Overcomplete tensor decomposition}

In this section, we give a polynomial-time algorithm for the following problem when $n \leqslant$ $d^{4 / 3} /($ polylog $d)$ :

Problem 5.1. Given an order-3 tensor $\mathbf{T}=\sum_{i=1}^{n} a_{i} \otimes a_{i} \otimes a_{i}$, where $a_{1}, \ldots, a_{n} \in \mathbb{R}^{d}$ are iid vectors sampled from $\mathcal{N}\left(0, \frac{1}{d} \mathrm{Id}\right)$, find vectors $b_{1}, \ldots, b_{n} \in \mathbb{R}^{n}$ such that for all $i \in[n]$,

$$
\left\langle a_{i}, b_{i}\right\rangle \geqslant 1-o(1)
$$

We give an algorithm that solves this problem, so long as the overcompleteness of the input tensor is bounded such that $n \ll d^{4 / 3} /$ polylog $d$.

Theorem 5.2. Given as input the tensor $\mathbf{T}=\sum_{i=1}^{n} a_{i} \otimes a_{i} \otimes a_{i}$ where $a_{i} \sim \mathcal{N}\left(0, \frac{1}{d} \operatorname{Id}_{d}\right)$ with $d \leqslant n \leqslant$ $d^{4 / 3} /$ polylog $d^{8}$ there is an algorithm which may run in time $\tilde{O}\left(n d^{1+\omega}\right)$ or $\tilde{O}\left(n d^{3.257}\right)$, where $d^{\omega}$ is the time to multiply two $d \times d$ matrices, which with probability $1-o(1)$ over the input $\mathbf{T}$ and the randomness of the algorithm finds unit vectors $b_{1}, \ldots, b_{n} \in \mathbb{R}^{d}$ such that for all $i \in[n]$,

$$
\left\langle a_{i}, b_{i}\right\rangle \geqslant 1-\tilde{O}\left(\frac{n^{3 / 2}}{d^{2}}\right) .
$$

\footnotetext{
${ }^{8}$ The lower bound $d \leqslant n$ on $n$, is a matter of technical convenience, avoiding separate concentration analyses and arithmetic in the undercomplete $(n<d)$ and overcomplete $(n \geqslant d)$ settings. Indeed, our algorithm still works in the undercomplete setting (tensor decomposition is easier in the undercomplete setting than the overcomplete one), but here other algorithms based on local search also work [AGJ15].
} 
We remark that this accuracy can be improved from $1-\tilde{O}\left(n^{3 / 2} / d^{2}\right)$ to an arbitrarily good precision using existing local search methods with local convergence guarantees-see Corollary 5.23.

As discussed in Section 2, to decompose the tensor $\sum_{i} a_{i}^{\otimes 6}$ (note we do not actually have access to this input!) there is a very simple tensor decomposition algorithm: sample a random $g \in \mathbb{R}^{d^{2}}$ and compute the matrix $\sum_{i}\left\langle g, a_{i}^{\otimes 2}\right\rangle\left(a_{i} a_{i}^{\top}\right)^{\otimes 2}$. With probability roughly $n^{-O(\varepsilon)}$ this matrix has (up to scaling) the form $\left(a_{i} a_{i}^{\top}\right)^{\otimes 2}+E$ for some $\|E\| \leqslant 1-\varepsilon$, and this is enough to recover $a_{i}$.

However, instead of $\sum_{i} a_{i}^{\otimes 6}$, we have only $\sum_{i, j}\left(a_{i} \otimes a_{j}\right)^{\otimes 3}$. Unfortunately, running the same algorithm on the latter input will not succeed. To see why, consider the extra terms $E^{\prime}:=\sum_{i \neq j}\left\langle g, a_{i} \otimes\right.$ $\left.a_{j}\right\rangle\left(a_{i} \otimes a_{j}\right)^{\otimes 2}$. Since $\left|\left\langle g, a_{i} \otimes a_{j}\right\rangle\right| \approx 1$, it is straightforward to see that $\left\|E^{\prime}\right\|_{F} \approx n$. Since the rank of $E^{\prime}$ is clearly $d^{2}$, even if we are lucky and all the eigenvalues have similar magnitudes, still a typical eigenvalue will be $\approx n / d \gg 1$, swallowing the $\sum_{i} a_{i}^{\otimes 6}$ term.

A convenient feature separating the signal terms $\sum_{i}\left(a_{i} \otimes a_{i}\right)^{\otimes 3}$ from the crossterms $\sum_{i \neq j}\left(a_{i} \otimes a_{j}\right)^{\otimes 3}$ is that the crossterms are not within the span of the $a_{i} \otimes a_{i}$. Although we cannot algorithmically access $\operatorname{Span}\left\{a_{i} \otimes a_{i}\right\}$, we have access to something almost as good: the unfolded input tensor, $T=$ $\sum_{i \in[n]} a_{i}\left(a_{i} \otimes a_{i}\right)^{\top}$. The rows of this matrix lie in $\operatorname{Span}\left\{a_{i} \otimes a_{i}\right\}$, and so for $i \neq j,\left\|T\left(a_{i} \otimes a_{i}\right)\right\| \gg\left\|T\left(a_{i} \otimes a_{j}\right)\right\|$. In fact, careful computation reveals that $\left\|T\left(a_{i} \otimes a_{i}\right)\right\| \geqslant \tilde{\Omega}(\sqrt{n} / d)\left\|T\left(a_{i} \otimes a_{j}\right)\right\|$.

The idea now is to replace $\sum_{i, j}\left\langle g, a_{i} \otimes a_{j}\right\rangle\left(a_{i} \otimes a_{j}\right)^{\otimes 2}$ with $\sum_{i, j}\left\langle g, T\left(a_{i} \otimes a_{j}\right)\right\rangle\left(a_{i} \otimes a_{j}\right)^{\otimes 2}$, now with $g \sim \mathcal{N}\left(0, \operatorname{Id}_{d}\right)$. As before, we are hoping that there is $i_{0}$ so that $\left\langle g, T\left(a_{i_{0}} \otimes a_{i_{0}}\right)\right\rangle \gg \max _{j \neq i_{0}}\left\langle g, T\left(a_{j} \otimes a_{j}\right)\right\rangle$. But now we also require $\left\|\sum_{i \neq j}\left\langle g, T\left(a_{i} \otimes a_{j}\right)\right\rangle\left(a_{i} \otimes a_{j}\right)\left(a_{i} \otimes a_{j}\right)^{\top}\right\| \ll\left\langle g, T\left(a_{i_{0}} \otimes a_{i_{0}}\right)\right\rangle \approx\left\|T\left(a_{i} \otimes a_{i}\right)\right\|$. If we are lucky and all the eigenvalues of this cross-term matrix have roughly the same magnitude (indeed, we will be lucky in this way), then we can estimate heuristically that

$$
\begin{aligned}
\left\|\sum_{i \neq j}\left\langle g, T\left(a_{i} \otimes a_{j}\right)\right\rangle\left(a_{i} \otimes a_{j}\right)\left(a_{i} \otimes a_{j}\right)^{\top}\right\| & \approx \frac{1}{d}\left\|\sum_{i \neq j}\left\langle g, T\left(a_{i} \otimes a_{j}\right)\right\rangle\left(a_{i} \otimes a_{j}\right)\left(a_{i} \otimes a_{j}\right)^{\top}\right\|_{F} \\
& \leqslant \frac{1}{d} \cdot \frac{\sqrt{n}}{d}\left|\left\langle g, T\left(a_{i_{0}} \otimes a_{i_{0}}\right)\right\rangle\right|\left\|\sum_{i \neq j}\left(a_{i} \otimes a_{j}\right)\left(a_{i} \otimes a_{j}\right)^{\top}\right\|_{F} \\
& \leqslant \frac{n^{3 / 2}}{d^{2}}\left|\left\langle g, T\left(a_{i_{0}} \otimes a_{i_{0}}\right)\right\rangle\right|,
\end{aligned}
$$

suggesting our algorithm will succed when $n^{3 / 2} \ll d^{2}$, which is to say $n \ll d^{4 / 3}$.

The following theorem, which formalizes the intuition above, is at the heart of our tensor decomposition algorithm.

Theorem 5.3. Let $a_{1}, \ldots, a_{n}$ be independent random vectors from $\mathcal{N}\left(0, \frac{1}{d} \operatorname{Id}_{d}\right)$ with $d \leqslant n \leqslant d^{4 / 3} /(\operatorname{polylog} d)$ and let $g$ be a random vector from $N\left(0, \mathrm{Id}_{d}\right)$. Let $\Sigma:=\mathbb{E}_{x \sim \mathcal{N}\left(0, \mathrm{Id}_{d}\right)}\left(x x^{\top}\right)^{\otimes 2}$ and let $R:=\sqrt{2} \cdot\left(\Sigma^{+}\right)^{1 / 2}$. Let $T=\sum_{i \in[n]} a_{i}\left(a_{i} \otimes a_{i}\right)^{\top}$. Define the matrix $M \in \mathbb{R}^{d^{2} \times d^{2}}$,

$$
M=\sum_{i, j \in[n]}\left\langle g, T\left(a_{i} \otimes a_{j}\right)\right\rangle \cdot\left(a_{i} \otimes a_{j}\right)\left(a_{i} \otimes a_{j}\right)^{\top} .
$$

With probability $1-o(1)$ over the choice of $a_{1}, \ldots, a_{n}$, for every polylog $d / \sqrt{d}<\varepsilon<1$, the spectral gap of $R M R$ is at least $\lambda_{2} / \lambda_{1} \leqslant 1-O(\varepsilon)$ and the top eigenvector $u \in \mathbb{R}^{d^{2}}$ of RMR satisfies, with probability $\tilde{\Omega}\left(1 / n^{O(\varepsilon)}\right)$ over the choice of $g$,

$$
\max _{i \in[n]}\left\langle R u, a_{i} \otimes a_{i}\right\rangle^{2} /\left(\|u\|^{2} \cdot\left\|a_{i}\right\|^{4}\right) \geqslant 1-\tilde{O}\left(\frac{n^{3 / 2}}{\varepsilon d^{2}}\right) .
$$


Moreover, with probability $1-o(1)$ over the choice of $a_{1}, \ldots, a_{n}$, for every polylog $d / \sqrt{d}<\varepsilon<1$ there are events $E_{1}, \ldots, E_{n}$ so that $\mathbb{P}_{g} E_{i} \geqslant \tilde{\Omega}\left(1 / n^{1+O(\varepsilon)}\right)$ for all $i \in[n]$ and when $E_{i}$ occurs, $\left\langle R u, a_{i} \otimes a_{i}\right\rangle^{2} /\|u\|^{2} \cdot\left\|a_{i}\right\|^{4} \geqslant$ $1-\tilde{O}\left(\frac{n^{3 / 2}}{\varepsilon d^{2}}\right)$.

We will eventually set $\varepsilon=1 / \log n$, which gives us a spectral algorithm for recovering a vector $\left(1-\tilde{O}\left(n / d^{3 / 2}\right)\right)$-correlated to some $a_{i}^{\otimes 2}$. Once we have a vector correlated with each $a_{i}^{\otimes 2}$, obtaining vectors close to the $a_{i}$ is straightforward. We will begin by proving this theorem, and defer the algorithmic details to section Section 5.4.

The rest of this section is organized as follows. In Section 5.1 we prove Theorem 5.3 using two core facts: the Gaussian vector $g$ is closer to some $a_{i}$ than to any other with good probability, and the noise term $\sum_{i \neq j}\left\langle g, T\left(a_{i} \otimes a_{j}\right)\right\rangle\left(a_{i} \otimes a_{j}\right)\left(a_{i} \otimes a_{j}\right)^{\top}$ is bounded in spectral norm. In Section 5.2 we prove the first of these two facts, and in Section 5.3 we prove the second. In Section 5.4, we give the full details of our tensor decomposition algorithm, then prove Theorem 5.2 using Theorem 5.3. Finally, Section C contains proofs of elementary or long-winded lemmas we use along the way.

\subsection{Proof of Theorem 5.3}

The strategy to prove Theorem 5.3 is to decompose the matrix $M$ into two parts $M=M_{\text {diag }}+M_{\text {cross }}$, one formed by diagonal terms $M_{\text {diag }}=\sum_{i \in[n]}\left\langle g, T\left(a_{i} \otimes a_{i}\right)\right\rangle \cdot\left(a_{i} \otimes a_{i}\right)\left(a_{i} \otimes a_{i}\right)^{\top}$ and one formed by cross terms $M_{\text {cross }}=\sum_{i \neq j}\left\langle g, T\left(a_{i} \otimes a_{j}\right)\right\rangle \cdot\left(a_{i} \otimes a_{j}\right)\left(a_{i} \otimes a_{j}\right)^{\top}$. We will use the fact that the top eigenvector $M_{\text {diag }}$ is likely to be correlated with one of the vectors $a_{j}^{\otimes 2}$, and also the fact that the spectral gap of $M_{\text {diag }}$ is noticeable.

The following two propositions capture the relevant facts about the spectra of $M_{\text {diag }}$ and $M_{\text {cross, }}$ and will be proven in Section 5.2 and Section 5.3 .

Proposition 5.4 (Spectral gap of diagonal terms). Let $R=\sqrt{2} \cdot\left(\left(\mathbb{E}\left(x x^{\top}\right)^{\otimes 2}\right)^{+}\right)^{1 / 2}$ for $x \sim \mathcal{N}\left(0, \operatorname{Id}_{d}\right)$. Let $a_{1}, \ldots, a_{n}$ be independent random vectors from $\mathcal{N}\left(0, \frac{1}{d} \mathrm{Id}_{d}\right)$ with $d \leqslant n \leqslant d^{2-\Omega(1)}$ and let $g \sim \mathcal{N}\left(0, \operatorname{Id}_{d}\right)$ be independent of all the others. Let $T:=\sum_{i \in[n]} a_{i}\left(a_{i} \otimes a_{i}\right)^{\top}$. Suppose $M_{\text {diag }}=\sum_{i \in[n]}\left\langle g, T a_{i}^{\otimes 2}\right\rangle \cdot\left(a_{i} a_{i}^{\top}\right)^{\otimes 2}$. Let also $v_{j}$ be such that $v_{j} v_{j}^{\top}=\left\langle g, T a_{j}^{\otimes 2}\right\rangle \cdot\left(a_{j} a_{j}^{\top}\right)^{\otimes 2}$. Then, with probability $1-o(1)$ over $a_{1}, \ldots, a_{n}$, for each $\varepsilon>$ polylog $d / \sqrt{d}$ and each $j \in[n]$, the event

$$
E_{j, \varepsilon} \stackrel{\text { def }}{=}\left\{\left\|R M_{\text {diag }} R-\varepsilon \cdot R v_{j} v_{j}^{\top} R\right\| \leqslant\left\|R M_{\text {diag }} R\right\|-(\varepsilon-\tilde{O}(\sqrt{n} / d)) \cdot\left\|R v_{j} v_{j}^{\top} R\right\|\right\}
$$

has probability at least $\tilde{\Omega}\left(1 / n^{1+O(\varepsilon)}\right)$ over the choice of $g$.

Second, we show that when $n \ll d^{4 / 3}$ the spectral norm of $M_{\text {cross }}$ is negligible compared to this spectral gap.

Proposition 5.5 (Bound on crossterms). Let $a_{1}, \ldots, a_{n}$ be independent random vectors from $\mathcal{N}\left(0, \frac{1}{d} \operatorname{Id}_{d}\right)$, and let $g$ be a random vector from $\mathcal{N}\left(0, \mathrm{Id}_{d}\right)$. Let $T:=\sum_{i \in[n]} a_{i}\left(a_{i} \otimes a_{i}\right)^{\top}$. Let $M_{\text {cross }}:=\sum_{i \neq j \in[n]}\left\langle g, T\left(a_{i} \otimes\right.\right.$ $\left.\left.a_{j}\right)\right\rangle a_{i} a_{i}^{\top} \otimes a_{j} a_{j}^{\top}$. Suppose $n \geqslant d$. Then with w.ov.p.,

$$
\left\|M_{\text {cross }}\right\| \leqslant \tilde{O}\left(\frac{n^{3}}{d^{4}}\right)^{1 / 2} .
$$


Using these two propositions we will conclude that the top eigenvector of $R M R$ is likely to be correlated with one of the vectors $a_{j}^{\otimes 2}$. We also need two simple concentration bounds; we defer the proof to the appendix.

Lemma 5.6. Let $a_{1}, \ldots, a_{n}$ be independently sampled vectors from $\mathcal{N}\left(0, \frac{1}{d} \operatorname{Id}_{d}\right)$, and let $g$ be sampled from $\mathcal{N}\left(0, \mathrm{Id}_{d}\right)$. Let $T=\sum_{i} a_{i}\left(a_{i} \otimes a_{i}\right)^{\top}$. Then with overwhelming probability, for every $j \in[n]$,

$$
\left|\left\langle g, T\left(a_{j} \otimes a_{j}\right)\right\rangle-\left\langle g, a_{j}\right\rangle\left\|a_{j}\right\|^{4}\right| \leqslant \tilde{O}\left(\frac{\sqrt{n}}{d}\right)
$$

Fact 5.7 (Simple version of Fact C.1). Let $x, y \sim \mathcal{N}\left(0, \frac{1}{d}\right.$ Id). With overwhelming probability, $\left|1-\|x\|^{2}\right| \leqslant$ $\tilde{O}(1 / \sqrt{d})$ and $\langle x, y\rangle^{2}=\tilde{O}(1 / d)$.

As a last technical tool we will need a simple claim about the fourth moment matrix of the multivariate Gaussian:

Fact 5.8 (simple version of Fact C.4). Let $\Sigma=\mathbb{E}_{x \sim \mathcal{N}\left(0, \mathrm{Id}_{d}\right)}\left(x x^{\top}\right)^{\otimes 2}$ and let $R=\sqrt{2}\left(\Sigma^{+}\right)^{1 / 2}$. Then $\|R\|=1$, and for any $v \in \mathbb{R}^{d}$,

$$
\|R(v \otimes v)\|_{2}^{2}=\left(1-\frac{1}{d+2}\right) \cdot\|v\|^{4} .
$$

We are prepared prove Theorem 5.3.

Proof of Theorem 5.3. Let $d \leqslant n \leqslant d^{4 / 3} /(\operatorname{polylog} d)$ for some polylog $d$ to be chosen later. Let $a_{1}, \ldots, a_{n}$ be independent random vectors from $\mathcal{N}\left(0, \frac{1}{d} \operatorname{Id}_{d}\right)$ and let $g \sim \mathcal{N}\left(0, \operatorname{Id}_{d}\right)$ be independent of the others. Let

$$
M_{\text {diag }}:=\sum_{i \in[n]}\left\langle g, T\left(a_{i} \otimes a_{i}\right)\right\rangle \cdot\left(a_{i} a_{i}^{\top}\right)^{\otimes 2} \quad \text { and } \quad M_{\text {cross }} \quad:=\sum_{i \neq j \in[n]}\left\langle g, T\left(a_{i} \otimes a_{j}\right)\right\rangle \cdot a_{i} a_{i}^{\top} \otimes a_{j} a_{j}^{\top} .
$$

Note that $M:=M_{\text {diag }}+M_{\text {cross }}$.

Proposition 5.5 implies that

$$
\mathbb{P}\left\{\left\|M_{\text {cross }}\right\| \leqslant \tilde{O}\left(n^{3 / 2} / d^{2}\right)\right\} \geqslant 1-d^{-\omega(1)} .
$$

Recall that $\Sigma=\mathbb{E}_{x \sim \mathcal{N}\left(0, I_{d}\right)}\left(x x^{\top}\right)^{\otimes 2}$ and $R=\sqrt{2} \cdot\left(\Sigma^{+}\right)^{1 / 2}$. By Proposition 5.4, with probability $1-o(1)$ over the choice of $a_{1}, \ldots, a_{n}$, each of the following events $E_{j, \varepsilon}$ for $j \in[n]$ and $\varepsilon>$ polylog $(d) / \sqrt{d}$ has probability at least $\tilde{\Omega}\left(1 / n^{1+O(\varepsilon)}\right)$ over the choice of $g$ :

$$
E_{j, \varepsilon}^{0}: \quad\left\|R\left(M_{\text {diag }}-\varepsilon\left\langle g, T a_{j}^{\otimes 2}\right\rangle\left(a_{j} a_{j}^{\top}\right)^{\otimes 2}\right) R\right\| \leqslant\left\|R M_{\text {diag }} R\right\|-(\varepsilon-\tilde{O}(\sqrt{n} / d)) \cdot\left(\left|\left\langle g, T a_{j}^{\otimes 2}\right\rangle\right| \cdot\left\|R a_{j}^{\otimes 2}\right\|^{2}\right) .
$$

Together with (5.1), with probability $1-o(1)$ over the choice of $a_{1}, \ldots, a_{n}$, each of the following events $E_{j, \varepsilon}^{*}$ has probability at least $\tilde{\Omega}\left(1 / n^{1+O(\varepsilon)}\right)-d^{-\omega(1)} \geqslant \tilde{\Omega}\left(1 / n^{1+O(\varepsilon)}\right)$ over the choice of $g$,

$$
\begin{aligned}
E_{j, \varepsilon}^{*}: \quad & \left\|R\left(M-\varepsilon\left\langle g, T a_{j}^{\otimes 2}\right\rangle\left(a_{j} a_{j}^{\top}\right)^{\otimes 2}\right) R\right\| \\
\leqslant & \left\|R \cdot M_{\mathrm{diag}} \cdot R\right\| \\
& \quad-(\varepsilon-\tilde{O}(\sqrt{n} / d)) \cdot\left(\left|\left\langle g, T a_{j}^{\otimes 2}\right\rangle\right| \cdot\left\|R a_{j}^{\otimes 2}\right\|^{2}\right)+\tilde{O}\left(n^{3 / 2} / d^{2}\right) \\
\leqslant & \|R \cdot M \cdot R\|-(\varepsilon-\tilde{O}(\sqrt{n} / d)) \cdot\left|\left\langle g, T a_{j}^{\otimes 2}\right\rangle\right| \cdot\left\|R a_{j}^{\otimes 2}\right\|^{2}+\tilde{O}\left(n^{3 / 2} / d^{2}\right) .
\end{aligned}
$$


Here, we used that $M=M_{\text {diag }}+M_{\text {cross }}$ and that $\left\|R \cdot M_{\text {cross }} \cdot R\right\| \leqslant\left\|M_{\text {cross }}\right\|$ as $\|R\| \leqslant 1$ (Fact 5.8).

By standard reasoning about the top eigenvector of a matrix with a spectral gap (recorded in Lemma A.3), the event $E_{j, \varepsilon}^{*}$ implies that the top eigenvector $u \in \mathbb{R}^{d^{2}}$ of $R \cdot M \cdot R$ satisfies

$$
\left\langle u, \frac{R a_{j}^{\otimes 2}}{\left\|R a_{j}^{\otimes 2}\right\|}\right\rangle^{2} \geqslant 1-\frac{\tilde{O}(\sqrt{n} / d)}{\varepsilon\left\|R a_{j}^{\otimes 2}\right\|^{2}}-\frac{\tilde{O}\left(n^{3 / 2} / d^{2}\right)}{\varepsilon\left\|R a_{j}^{\otimes 2}\right\|^{2}\left|\left\langle g, T a_{j}^{\otimes 2}\right\rangle\right|} .
$$

Since $\left\|R a_{j}{ }^{\otimes 2}\right\|^{2} \geqslant \Omega\left(\left\|a_{j}\right\|^{4}\right)$ (by Fact 5.8), and since $\left\|a_{j}\right\| \geqslant 1-\tilde{O}(1 / \sqrt{d})$ (by Fact 5.7),

$$
\geqslant 1-\tilde{O}\left(\frac{\sqrt{n}}{\varepsilon d}\right)-\frac{\tilde{O}\left(n^{3 / 2} / d^{2}\right)}{\varepsilon \cdot\left|\left\langle g, T a_{j}^{\otimes 2}\right\rangle\right|}
$$

Now, by Lemma 5.6 we have that for all $j \in[n],\left|\left\langle g, T a_{j}^{\otimes 2}\right\rangle-\left\langle g, a_{j}\right\rangle\left\|a_{j}\right\|^{4}\right| \leqslant \tilde{O}(\sqrt{n} / d)$ with probability $1-n^{-\omega(1)}$. By standard concentration (see Fact C.1 for a proof) $\left|\left\langle g, a_{j}\right\rangle\right|\left|a_{j} \|^{4}-1\right| \leqslant \tilde{O}(1 / \sqrt{d}$ ) for all $j \in[n]$ with probability $1-n^{-\omega(1)}$. Therefore with overwhelming probability, the final term is bounded by $\tilde{O}\left(n^{3 / 2} / \varepsilon d^{2}\right)$. A union bound now gives the desired conclusion.

Finally, we give a bound on the spectral gap. We note that the second eigenvector $w$ has $\langle u, w\rangle=0$, and therefore

$$
\left\langle w, \frac{R a_{j}^{\otimes 2}}{\left\|R a_{j}^{\otimes 2}\right\|}\right\rangle=\left\langle w, \frac{R a_{j}^{\otimes 2}}{\left\|R a_{j}^{\otimes 2}\right\|}-u\right\rangle \leqslant\left\|\frac{R a_{j}^{\otimes 2}}{\left\|R a_{j}^{\otimes 2}\right\|}-u\right\| \leqslant \tilde{O}\left(n^{3 / 2} / \varepsilon d^{2}\right) .
$$

Thus, using our above bound on $\left\|R\left(M-\varepsilon\left\langle g, T a_{j}^{\otimes 2}\right\rangle\left(a_{j} a_{j}^{\top}\right)^{\otimes 2}\right) R\right\|$ and the concentration bounds we have already applied for $\left\|a_{j}\right\|,\left\langle g, T a_{j}^{\otimes 2}\right\rangle$, and $\left\|R a_{j}^{\otimes 2}\right\|$, we have that

$$
\begin{aligned}
\lambda_{2}(R M R) & =w^{\top} R M R w \\
& =w^{\top} R\left(M-\varepsilon\left\langle g, T a_{j}^{\otimes 2}\right\rangle \cdot\left(a_{j} a_{j}^{\top}\right)^{\otimes 2}\right) R w+\varepsilon\left\langle g, T a_{j}^{\otimes 2}\right\rangle\left\langle w, R a_{j}^{\otimes 2}\right\rangle^{2} \\
& \leqslant\left\|R\left(M-\varepsilon\left\langle g, T a_{j}^{\otimes 2}\right\rangle \cdot\left(a_{j} a_{j}^{\top}\right)^{\otimes 2}\right) R\right\|+\tilde{O}\left(n^{3 / 2} / \varepsilon d^{2}\right) \\
& \leqslant 1-\tilde{O}(\varepsilon)+\tilde{O}\left(n^{3 / 2} / \varepsilon d^{2}\right) .
\end{aligned}
$$

We conclude that the above events also imply that $\lambda_{2}(R M R) / \lambda_{1}(R M R) \leqslant 1-O(\varepsilon)$.

\subsection{Spectral gap for diagonal terms: proof of Proposition 5.4}

We now prove that the signal matrix, when preconditioned by $R$, has a noticeable spectral gap:

Proposition (Restatement of Proposition 5.4). Let $R=\sqrt{2} \cdot\left(\left(\mathbb{E}\left(x x^{\top}\right)^{\otimes 2}\right)^{+}\right)^{1 / 2}$ for $x \sim \mathcal{N}\left(0, \operatorname{Id}_{d}\right)$. Let $a_{1}, \ldots, a_{n}$ be independent random vectors from $\mathcal{N}\left(0, \frac{1}{d} \operatorname{Id}_{d}\right)$ with $d \leqslant n \leqslant d^{2-\Omega(1)}$ and let $g \sim \mathcal{N}\left(0, \operatorname{Id}_{d}\right)$ be independent of all the others. Let $T:=\sum_{i \in[n]} a_{i}\left(a_{i} \otimes a_{i}\right)^{\top}$. Suppose $M_{\text {diag }}=\sum_{i \in[n]}\left\langle g, T a_{i}^{\otimes 2}\right\rangle \cdot\left(a_{i} a_{i}^{\top}\right)^{\otimes 2}$. Let also $v_{j}$ be such that $v_{j} v_{j}^{\top}=\left\langle g, T a_{j}^{\otimes 2}\right\rangle \cdot\left(a_{j} a_{j}^{\top}\right)^{\otimes 2}$. Then, with probability $1-o(1)$ over $a_{1}, \ldots, a_{n}$, for each $\varepsilon>$ polylog $d / \sqrt{d}$ and each $j \in[n]$, the event

$$
E_{j, \varepsilon} \stackrel{\text { def }}{=}\left\{\left\|R M_{\text {diag }} R-\varepsilon \cdot R v_{j} v_{j}^{\top} R\right\| \leqslant\left\|R M_{\text {diag }} R\right\|-(\varepsilon-\tilde{O}(\sqrt{n} / d)) \cdot\left\|R v_{j} v_{j}^{\top} R\right\|\right\}
$$

has probability at least $\tilde{\Omega}\left(1 / n^{1+O(\varepsilon)}\right)$ over the choice of $g$. 
The proof has two parts. First we show that for $a_{1}, \ldots, a_{n} \sim \mathcal{N}\left(0, \operatorname{Id}_{d}\right)$ the matrix $P:=$ $\sum_{i \in[n]}\left(a_{i} a_{i}^{\top}\right)^{\otimes 2}$ has tightly bounded spectral norm when preconditioned with $R$ : more precisely, that $\|R P R\| \leqslant 1+\tilde{O}\left(n / d^{3 / 2}\right)$.

Lemma 5.9. Let $a_{1}, \ldots, a_{n} \sim \mathcal{N}\left(0, \frac{1}{d} \operatorname{Id}_{d}\right)$ be independent random vectors with $d \leqslant n$. Let $R:=\sqrt{2}$. $\left(\left(\mathbb{E}\left(a a^{\top}\right)^{\otimes 2}\right)^{+}\right)^{1 / 2}$ for $a \sim \mathcal{N}\left(0, \operatorname{Id}_{d}\right)$. For $S \subseteq[n]$, let $P_{S}=\sum_{i \in S}\left(a_{i} a_{i}^{\top}\right)^{\otimes 2}$ and let $\Pi_{S}$ be the projector into the subspace spanned by $\left\{R a_{i}^{\otimes 2} \mid i \in S\right\}$. Then, with probability $1-o(1)$ over the choice of $a_{1}, \ldots, a_{n}$,

$$
\forall S \subseteq[n] . \quad\left(1-\tilde{O}\left(n / d^{3 / 2}\right)\right) \cdot \Pi_{S} \leq R P_{S} R \leq\left(1+\tilde{O}\left(n / d^{3 / 2}\right)\right) \cdot \Pi_{S} .
$$

Remark 5.10. In [GM15, Lemma 5] a similar lemma to this one is proved in the context of the SoS proof system. However, since Ge and Ma leverage the full power of the SoS algorithm their proof goes via a spectral bound on a different (but related) matrix. Since our algorithm avoids solving an SDP we need a bound on this matrix in particular.

The proof of Lemma 5.9 proceeds by standard spectral concentration for tall matrices with independent columns (here the columns are $R a_{i}^{\otimes 2}$ ). The arc of the proof is straightforward but it involves some bookkeeping; we have deferred it to Section C.0.4.

We also need the following lemma on the concentration of some scalar random variables involving $R$; the proof is straightforward by finding the eigenbasis of $R$ and applying standard concentration, and it is deferred to the appendix.

Lemma 5.11. Let $a_{1}, \ldots, a_{n} \sim \mathcal{N}\left(0, \frac{1}{d} \operatorname{Id}_{d}\right)$. Let $\Sigma, R$ be as in Fact 5.8. Let $u_{i}=a_{i} \otimes a_{i}$. With overwhelming probability, every $j \in[n]$ satisfies $\sum_{i \neq j}\left\langle u_{j}, R^{2} u_{i}\right\rangle^{2}=\tilde{O}\left(n / d^{2}\right)$ and $\left|1-\left\|R u_{j}\right\|^{2}\right| \leqslant \tilde{O}(1 / \sqrt{d})$.

The next lemma is the linchpin of the proof of Proposition 5.4: one of the inner products $\left\langle g, T a_{j}{ }^{\otimes 2}\right\rangle$, is likely to be $\mathrm{a} \approx(1+1 / \log (n))$-factor larger than the maximum of the inner products $\left\langle g, T a_{i}{ }^{\otimes 2}\right\rangle$ over $i \neq j$. Together with standard linear algebra these imply that the matrix $M_{\text {diag }}=$ $\sum_{i \in[n]}\left\langle g, T a_{i}^{\otimes 2}\right\rangle\left(a_{i} a_{i}^{\top}\right)^{\otimes 2}$ has top eigenvector highly correlated or anticorrelated with some $a_{i}$.

Lemma 5.12. Let $a_{1}, \ldots, a_{n} \in \mathbb{R}^{d}$ be independent random vectors from $\mathcal{N}\left(0, \frac{1}{d} \operatorname{Id}_{d}\right)$, and let $g$ be a random vector from $\mathcal{N}\left(0, \operatorname{Id}_{d}\right)$. Let $T=\sum_{i \in[n]} a_{i}\left(a_{i} \otimes a_{i}\right)^{\top}$. Let $\varepsilon>0$ and $j \in[n]$. Then with overwhelming probability over $a_{1}, \ldots, a_{n}$, the following event $\hat{E}_{j, \varepsilon}$ has probability $1 / n^{1+O(\varepsilon)+\tilde{O}(1 / \sqrt{d})}$ over the choice of $g$,

$$
\hat{E}_{j, \varepsilon}=\left\{\left\langle g, T a_{j}^{\otimes 2}\right\rangle \geqslant(1+\varepsilon)(1-\tilde{O}(1 / \sqrt{d})) \cdot \max _{i \neq j}\left|\left\langle g, T a_{i}^{\otimes 2}\right\rangle\right|\right\} .
$$

Now we can prove Proposition 5.4.

Proof of Proposition 5.4. Let $u_{i}:=a_{i}^{\otimes 2}$. Fix $j \in[n]$. We begin by showing a lower bound on the spectral norm $\left\|R M_{\text {diag }} R\right\|$.

$$
\begin{aligned}
\left\|R M_{\text {diag }} R\right\| & =\max _{\|v\|=1}\left|\left\langle v, R M_{\text {diag }} R v\right\rangle\right| \\
& \geqslant \frac{\left\langle R u_{j},\left(R M_{\text {diag }} R\right) R u_{j}\right\rangle}{\left\|R u_{j}\right\|^{2}} \\
& =\frac{1}{\left\|R u_{j}\right\|^{2}}\left(\left\langle g, T u_{j}\right\rangle\left\|R u_{j}\right\|^{4}+\left\langle R u_{j}, \sum_{i \neq j}\langle g, T\rangle R u_{i} u_{i}^{\top} R \cdot R u_{j}\right\rangle\right)
\end{aligned}
$$


From Lemma 5.12, the random vector $g$ is closer to $T u_{j}$ than to all $T u_{i}$ for $i \neq j, i \in[n]$ with reasonable probability. More concretely there is some polylog $d$ so that as long as $\varepsilon>$ polylog $d / \sqrt{d}$ there is some $\alpha=\Theta(\varepsilon)$ with $1-\varepsilon=1 /\left[(1+\alpha)\left(1-\tilde{O}\left(d^{-1 / 2}\right)\right)\right]$ so that with w.ov.p. over $a_{1}, \ldots, a_{n}$ the following event (a direct consequence of $\hat{E}_{j, \varepsilon}$ ) has probability $\tilde{\Omega}\left(1 / n^{1+O(\alpha)+\tilde{O}\left(d^{-1 / 2}\right)}\right)=\tilde{\Omega}\left(1 / n^{1+O(\varepsilon)}\right)$ over $g$ :

$$
-(1-\varepsilon)\left|\left\langle g, T u_{j}\right\rangle\right| \cdot\left(\sum_{i \neq j} R u_{i} u_{i}^{\top} R\right) \leq \sum_{i \neq j}\left\langle g, T u_{i}\right\rangle \cdot R u_{i} u_{i}^{\top} R \leq(1-\varepsilon)\left|\left\langle g, T u_{j}\right\rangle\right| \cdot\left(\sum_{i \neq j} R u_{i} u_{i}^{\top} R\right) .
$$

When (5.2) occurs,

$$
\begin{aligned}
\left\|R M_{\text {diag }} R\right\| & \geqslant \frac{1}{\left\|R u_{j}\right\|^{2}}\left(\left|\left\langle g, T u_{j}\right\rangle\left\|R u_{j}\right\|^{4}-(1-\varepsilon)\right|\left\langle g, T u_{j}\right\rangle \mid\left\langle R u_{j}, \sum_{i \neq j} R u_{i} u_{i}^{\top} R \cdot R u_{j}\right\rangle\right) \\
& =\frac{\left|\left\langle g, T u_{j}\right\rangle\right| \mid}{\left\|R u_{j}\right\|^{2}}\left(\left\|R u_{j}\right\|^{4}-(1-\varepsilon) \sum_{i \neq j}\left\langle u_{j}, R^{2} u_{i}\right\rangle^{2}\right) \\
& \geqslant \frac{\left|\left\langle g, T u_{j}\right\rangle\right|\left(1-\tilde{O}(1 / \sqrt{d})-(1-\varepsilon) \tilde{O}\left(n / d^{2}\right)\right)}{1+\tilde{O}(1 / \sqrt{d})} \quad \text { w.ov.p. over } a_{1}, \ldots, a_{n} \text { (Lemma 5.11) } \\
& \geqslant\left|\left\langle g, T u_{j}\right\rangle\right| \cdot\left(1-\eta_{\text {norm }}\right),
\end{aligned}
$$

where we have chosen some $0 \leqslant \eta_{\text {norm }} \leqslant \tilde{O}(1 / \sqrt{d})+\tilde{O}\left(n / d^{2}\right)$ (since for any $\left.x \in \mathbb{R},(1+x)(1-x) \leqslant 1\right)$.

Next we exhibit an upper bound on $\left\|R M_{\text {diag }} R-\varepsilon\left\langle g, T u_{j}\right\rangle R u_{j} u_{j}^{\top} R\right\|$. Again when (5.2) occurs,

$$
\begin{aligned}
& \left\|R M_{\text {diag }} R-\varepsilon\left\langle g, T u_{j}\right\rangle R u_{j} u_{j}^{\top} R\right\| \\
& \quad=\left\|(1-\varepsilon)\left\langle g, T u_{j}\right\rangle R u_{j} u_{j}^{\top} R+\sum_{i \neq j}\left\langle g, T u_{i}\right\rangle R u_{i} u_{i}^{\top} R\right\| \\
& \quad \leqslant(1-\varepsilon)\left|\left\langle g, T u_{j}\right\rangle\right|\left\|\sum_{i \in[n]} R u_{i} u_{i}^{\top} R\right\| \quad \text { when (5.2) occurs } \\
& \quad \leqslant(1-\varepsilon)\left|\left\langle g, T u_{j}\right\rangle\right|\left(1+\tilde{O}\left(n / d^{1.5}\right)\right) \quad \text { w.p. } 1-o(1) \text { over } a_{1}, \ldots, a_{n} \text { by Lemma } 5.9 \\
& \quad \leqslant(1-\varepsilon)\left|\left\langle g, T u_{j}\right\rangle\right|\left(1+\eta_{\text {gap }}\right)
\end{aligned}
$$

where we have chosen some $0 \leqslant \eta_{\text {gap }} \leqslant \tilde{O}\left(n / d^{1.5}\right)$.

Putting together (5.3) and (5.5) with our bounds on $\eta_{\text {norm }}$ and $\eta_{\text {gap }}$ and recalling the conditions on (5.2), we have shown that

$$
\begin{gathered}
\underset{a_{1}, \ldots, a_{n}}{\mathbb{P}}\left\{\underset{g}{\mathbb{P}}\left\{\left\|R M_{\text {diag }} R-\varepsilon\left\langle g, T u_{j}\right\rangle R u_{j} u_{j}^{\top} R\right\| \leqslant\left\|R M_{\text {diag }} R\right\|-(\varepsilon-\tilde{O}(\sqrt{n} / d)) \cdot\left|\left\langle g, T u_{j}\right\rangle\right| \cdot\left\|R u_{j}\right\|^{2}\right\}\right. \\
\left.\geqslant \tilde{\Omega}\left(1 / n^{1+O(\varepsilon)}\right)\right\} \geqslant 1-o(1) .
\end{gathered}
$$

This concludes the argument.

We now turn to proving that with reasonable probability, $g$ is closer to some $T a_{j}^{\otimes 2}$ than all others. 
Proof of Lemma 5.12. To avoid proliferation of indices, without loss of generality fix $j=1$. We begin by expanding the expression $\left\langle g, T a_{i}^{\otimes 2}\right\rangle$,

$$
\left\langle g, T a_{i}^{\otimes 2}\right\rangle=\sum_{\ell \in[n]}\left\langle g, a_{\ell}\right\rangle\left\langle a_{\ell}, a_{i}\right\rangle^{2}=\left\|a_{i}\right\|^{4}\left\langle g, a_{i}\right\rangle+\sum_{\ell \neq i}\left\langle g, a_{\ell}\right\rangle\left\langle a_{\ell}, a_{i}\right\rangle^{2} .
$$

The latter sum is bounded by

$$
\left|\sum_{\ell \neq i}\left\langle g, a_{\ell}\right\rangle\left\langle a_{\ell}, a_{i}\right\rangle^{2}\right| \leqslant \tilde{O}\left(\frac{\sqrt{n}}{d}\right),
$$

with overwhelming probability for all $i$ and choices of $g$; this follows from a Bersntein bound, given in Lemma 5.6.

For ease of notation, let $\hat{a}_{i} \stackrel{\text { def }}{=} a_{i} /\left\|a_{i}\right\|_{2}$. We conclude from Fact 5.7 that with overwhelming probability, $1-\tilde{O}(1 / \sqrt{d}) \leqslant\left\|a_{i}\right\|_{2} \leqslant 1+\tilde{O}(1 / \sqrt{d})$ for all $i \in[n]$. Thus $\left\|a_{i}\right\|_{2}$ is roughly equal for all $i$, and we may direct our attention to $\left\langle g, \hat{a}_{i}\right\rangle$.

Let $\mathcal{G}_{1}$ be the event that $\sqrt{2 \alpha} \log ^{1 / 2} n \leqslant\left|\left\langle g, \hat{a}_{1}\right\rangle\right| \leqslant d^{1 / 4}$ for some $\alpha \leqslant d^{1 / 2-\Omega(1)}$ to be chosen later. We note that $\left\langle g, \hat{a}_{1}\right\rangle$ is distributed as a standard gaussian, and that $g$ is independent of $a_{1}, \ldots, a_{n}$. Thus, we can use standard tail estimates on univariate Gaussians (Lemma A.4) to conclude that

$$
\mathbb{P}\left(\left|\left\langle g, \hat{a}_{1}\right\rangle\right| \geqslant \sqrt{2 \alpha} \log ^{1 / 2} n\right)=\tilde{\Theta}\left(n^{-\alpha}\right) \text { and } \mathbb{P}\left(\left|\left\langle g, \hat{a}_{1}\right\rangle\right| \geqslant d^{1 / 4}\right)=\Theta\left(\frac{\exp (-\sqrt{d} / 2)}{d^{1 / 4}}\right) \text {. }
$$

So by a union bound, $\mathbb{P}\left(\mathcal{G}_{1}\right) \geqslant \tilde{\Omega}\left(n^{-\alpha}\right)-O\left(e^{-d^{1 / 2} / 3}\right)=\tilde{\Omega}\left(n^{-\alpha}\right)$.

Now, we must obtain an estimate for the probability that all other inner products with $g$ are small. Let $\mathcal{G}_{i>1}$ be the event that $\left|\left\langle g, \hat{a}_{i}\right\rangle\right| \leqslant \sqrt{(2+\rho)} \log ^{1 / 2} n$ for all $i \in[n], i>1$ and for some $\rho$ to be chosen later. We will show that conditioned on $\mathcal{G}_{1}, \mathcal{G}_{i>1}$ occurs with probability $1-O\left(n^{1-(2+\rho) / 2}\right)$. Define $g_{1}:=\left\langle g, \hat{a}_{1}\right\rangle \hat{a}_{1}$ to be the component of $g$ parallel to $a_{1}$, let $g_{\perp}:=g-g_{1}$ be the component of $g$ orthogonal to $\hat{a}_{1}$, and similarly let $\hat{a}_{2}^{\perp}, \ldots, \hat{a}_{n}^{\perp}$ be the components of $\hat{a}_{2}, \ldots, \hat{a}_{n}$ orthogonal to $a_{1}$. Because $g_{\perp}$ is independent of $g_{1}$, even conditioned on $\mathcal{G}_{1}$ we may apply the standard tail bound for univariate Gaussians (Lemma A.4), concluding that for all $i>1$,

$$
\mathbb{P}\left(\left|\left\langle g_{\perp}, \hat{a}_{i}\right\rangle\right| \geqslant \sqrt{(2+\rho)} \log ^{1 / 2} n \mid \mathcal{G}_{1}\right)=\tilde{\Theta}\left(n^{-(2+\rho) / 2}\right) .
$$

Thus, a union bound over $i \neq 1$ allows us to conclude that conditioned on $\mathcal{G}_{1}$, with probability $1-\tilde{O}\left(n^{-\rho / 2}\right)$ every $i \in[n]$ with $i>1$ has $\left|\left\langle g_{\perp}, \hat{a}_{i}^{\perp}\right\rangle\right| \leqslant \sqrt{(2+\rho)} \log ^{1 / 2} n$.

On the other hand, let $\hat{a}_{2}^{\|}, \ldots, \hat{a}_{n}^{\|}$be the components of the $\hat{a}_{i}$ parallel to $\hat{a}_{1}$. We compute the projection of $\hat{a}_{i}$ onto $\hat{a}_{1}$. With overwhelming probability,

$$
\begin{aligned}
\left\langle\hat{a}_{1}, \hat{a}_{i}\right\rangle & =\frac{\left\langle a_{1}, a_{i}\right\rangle}{\left\|a_{1}\right\|_{2} \cdot\left\|a_{i}\right\|_{2}} \\
& =(1 \pm \tilde{O}(1 / \sqrt{d})) \cdot\left\langle a_{1}, a_{i}\right\rangle \quad \text { w.ov.p. by }\left\|a_{i}\right\|,\left\|a_{1}\right\|=1 \pm \tilde{O}(1 / \sqrt{d})(\text { Fact 5.7) } \\
& =(1 \pm \tilde{O}(1 / \sqrt{d})) \cdot \tilde{O}(1 / \sqrt{d}) \quad \text { w.ov.p. by }\left\langle a_{1}, a_{i}\right\rangle=\tilde{O}(1 / \sqrt{d})(\text { Fact 5.7), }
\end{aligned}
$$

Thus w.ov.p.,

$$
\left\langle g_{1}, \hat{a}_{i}^{\|}\right\rangle=\left\langle g, \hat{a}_{1}\right\rangle \cdot\left\langle\hat{a}_{1}, \hat{a}_{i}\right\rangle \leqslant\left\langle g, \hat{a}_{1}\right\rangle \cdot \tilde{O}(1 / \sqrt{d}),
$$


for all $i \in[n]$. Now we can analyze $\mathcal{G}_{i>1}$. Taking a union bound over the overwhelmingly probable events (including $\left\|a_{i}\right\| \leqslant 1+\tilde{O}(1 / \sqrt{d})$ ) and the event that $\left\langle g_{\perp}, a_{i}\right\rangle$ is small for all $i$, we have that with probability $1-\tilde{O}\left(n^{-\rho / 2}\right)$, for every $i \in[n]$ with $i>1$,

$$
\begin{aligned}
\left|\left\langle g, \hat{a}_{i}\right\rangle\right| & \leqslant\left|\left\langle g_{\perp}, \hat{a}_{i}\right\rangle\right|+\left|\left\langle g_{1}, \hat{a}_{i}\right\rangle\right| \\
& \sqrt{(2+\rho)} \log ^{1 / 2} n+\tilde{O}(1 / \sqrt{d}) \cdot\left\langle g, \hat{a}_{1}\right\rangle \\
& \leqslant \sqrt{(2+\rho)} \log ^{1 / 2} n+\tilde{O}\left(1 / d^{1 / 4}\right) .
\end{aligned}
$$

We conclude that

$$
\begin{aligned}
\mathbb{P}\left(\mathcal{G}_{1}, \mathcal{G}_{i>1}\right) & =\mathbb{P}\left(\mathcal{G}_{i>1} \mid \mathcal{G}_{1}\right) \cdot \mathbb{P}\left(\mathcal{G}_{1}\right) \\
& \geqslant\left(1-O\left(n^{-\rho / 2}\right)\right) \cdot \tilde{\Omega}\left(n^{-\alpha}\right)
\end{aligned}
$$

Setting $\rho=2 \frac{\log \log n}{\log n}$ and $\alpha=(1+\varepsilon)^{2}(1+\log \log n / \log n+\tilde{O}(1 / \sqrt{d}))$, the conclusion follows.

\subsection{Bound for cross terms: proof of Proposition 5.5}

We proceed to the bound on the cross terms $M_{\text {cross }}$.

Proposition (Restatement of Proposition 5.5). Let $a_{1}, \ldots, a_{n}$ be independent random vectors from $\mathcal{N}\left(0, \frac{1}{d} \operatorname{Id}_{d}\right)$, and let $g$ be a random vector from $\mathcal{N}\left(0, \operatorname{Id}_{d}\right)$. Let $T:=\sum_{i \in[n]} a_{i}\left(a_{i} \otimes a_{i}\right)^{\top}$. Let $M_{\text {cross }}:=$ $\sum_{i \neq j \in[n]}\left\langle g, T\left(a_{i} \otimes a_{j}\right)\right\rangle a_{i} a_{i}^{\top} \otimes a_{j} a_{j}^{\top}$. Suppose $n \geqslant d$. Then with w.ov.p.,

$$
\left\|M_{\text {cross }}\right\| \leqslant \tilde{O}\left(\frac{n^{3}}{d^{4}}\right)^{1 / 2} .
$$

The proof will use two iterations of Matrix Rademacher bounds. The first step will be to employ a classical decoupling inequality that has previously been used in a tensor decomposition context [GM15].

Theorem 5.13 (Special Case of Theorem 1 in [dlPMS95]). Let $\left\{s_{i}\right\},\left\{t_{i}\right\}$ be independent iid sequences of random signs. Let $\left\{M_{i j}\right\}$ be a family of matrices. There is a universal constant $C$ so that for every $t>0$,

$$
\mathbb{P}\left(\left\|\sum_{i \neq j} s_{i} s_{j} M_{i j}\right\|_{o p}>t\right) \leqslant C \cdot \mathbb{P}\left(C\left\|\sum_{i \neq j} s_{i} t_{j} M_{i j}\right\|_{o p}>t\right) .
$$

Once the simplified cross terms are decoupled, we can use a matrix Rademacher bound on one set of signs.

Theorem 5.14 (Adapted from Theorem 4.1.1 in [Tro12] ${ }^{9}$ ). Consider a finite sequence $\left\{M_{i}\right\}$ of fixed $m \times m$ Hermitian matrices. Let $s_{i}$ be a sequence of independent sign variables. Let $\sigma^{2}:=\left\|\sum_{i} M_{i}^{2}\right\|$. Then for every $t \geqslant 0$,

$$
\mathbb{P}\left(\left\|\sum_{i} s_{i} M_{i}\right\|_{o p} \geqslant t\right) \leqslant 2 m \cdot e^{-t^{2} / 2 \sigma^{2}} .
$$

\footnotetext{
${ }^{9}$ We remark that Tropp's bound is phrased in terms of $\lambda_{\max } \sum_{i} s_{i} M_{i}$. Since $\lambda_{\max } \sum_{i} s_{i} M_{i}=\lambda_{\min } \sum_{i}-s_{i} M_{i}$, and the distribution of $s_{i} M_{i}$ is negation-invariant, the result we state here follows from an easy union bound.
} 
Also,

$$
\mathbb{E}\left\|\sum_{i} s_{i} M_{i}\right\| \leqslant \sqrt{8 \sigma^{2} \log d} .
$$

Corollary 5.15. Let $s_{1}, \ldots, s_{n}$ be independent signs in $\{-1,1\}$. Let $A_{1}, \ldots, A_{n}$ and $B_{1}, \ldots, B_{n}$ be Hermetian matrices. Then w.ov.p.,

$$
\left\|\sum_{i} s_{i} \cdot A_{i} \otimes B_{i}\right\| \leqslant \tilde{O}\left(\max _{i}\left\|B_{i}\right\| \cdot\left\|\sum_{i} A_{i}^{2}\right\|^{1 / 2}\right) .
$$

Proof. We use a matrix Rademacher bound and standard manipulations:

$$
\begin{aligned}
\left\|\sum_{i} s_{i} \cdot A_{i} \otimes B_{i}\right\| & \stackrel{\text { w.ov.p. }}{\leqslant} \tilde{O}\left(\left\|\sum_{i} A_{i}^{2} \otimes B_{i}^{2}\right\|\right)^{1 / 2} \\
& \leqslant \tilde{O}\left(\left\|\sum_{i}\right\| B_{i}\left\|^{2} \cdot\left(A_{i}^{2} \otimes \mathrm{Id}\right)\right\|\right)^{1 / 2} \text { since } A_{i}^{2} \text { is PSD for all } i \\
& \leqslant \tilde{O}\left(\max _{i}\left\|B_{i}\right\|^{2} \cdot\left\|\sum_{i} A_{i}^{2}\right\|\right)^{1 / 2} \text { since } A_{i}^{2} \otimes \operatorname{Id} \text { is PSD for all } i .
\end{aligned}
$$

We also need a few further concentration bounds on matrices which will come up as parts of $M_{\text {cross }}$. These can be proved by standard inequalities for sums of independent matrices.

Lemma 5.16 (Restatement of Fact C.2 and Lemma C.3). Let $a_{1}, \ldots, a_{n}$ be independent from $\mathcal{N}\left(0, \frac{1}{d} \operatorname{Id}_{d}\right)$ with $n \geqslant d$ polylog $(d)$. With overwhelming probability, $\tilde{\Omega}(n / d) \cdot \operatorname{Id} \leq \sum_{i \in[n]} a_{i} a_{i}^{\top} \leq \tilde{O}(n / d) \cdot I d$. Additionally, if $g \sim \mathcal{N}\left(0, \mathrm{Id}_{d}\right)$ is independent of the rest, for every $j \in[n]$ w.ov.p.

$$
\left\|\sum_{\substack{i \in[n] \\ i \neq j}}\left\langle g, a_{i}\right\rangle\right\| a_{i}\left\|^{2}\left\langle a_{i}, a_{j}\right\rangle \cdot a_{i} a_{i}^{\top}\right\| \leqslant \tilde{O}\left(n / d^{2}\right)^{1 / 2} .
$$

Proof of Proposition 5.5. We expand $M_{\text {cross }}$ :

$$
\begin{aligned}
M_{\text {cross }} & =\sum_{i \neq j}\left\langle g, T\left(a_{i} \otimes a_{j}\right)\right\rangle \cdot a_{i} a_{i}^{\top} \otimes a_{j} a_{j}^{\top} \\
& =\sum_{i \neq j}\left(\sum_{\ell \in[n]}\left\langle a_{\ell}, a_{i}\right\rangle\left\langle a_{\ell}, a_{j}\right\rangle\left\langle g, a_{\ell}\right\rangle\right) \cdot a_{i} a_{i}^{\top} \otimes a_{j} a_{j}^{\top} .
\end{aligned}
$$

Since the joint distribution of $\left(a_{1}, \ldots, a_{n}\right)$ is identical to that of $\left(s_{1} a_{1}, \ldots, s_{n} a_{n}\right)$, this is distributed identically to

$$
M_{\text {cross }}^{\prime}=\sum_{\ell \in[n]} \sum_{i \neq j} s_{i} s_{j} s_{\ell}\left\langle g, a_{\ell}\right\rangle\left\langle a_{\ell}, a_{i}\right\rangle\left\langle a_{\ell}, a_{j}\right\rangle \cdot a_{i} a_{i}^{\top} \otimes a_{j} a_{j}^{\top},
$$




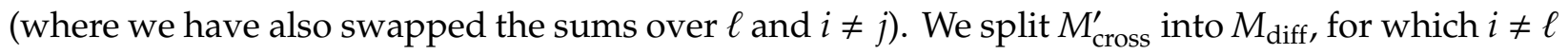
and $j \neq \ell$, and $M_{\text {same, }}$, for which $\ell=i$ or $\ell=j$, and bound the norm of each of these sums separately. We begin with $M_{\text {same }}$.

$$
M_{\text {same }} \stackrel{\text { def }}{=} \sum_{i \neq j} s_{i}^{2} s_{j}\left\langle g, a_{i}\right\rangle\left\langle a_{i}, a_{i}\right\rangle\left\langle a_{i}, a_{j}\right\rangle \cdot a_{i} a_{i}^{\top} \otimes a_{j} a_{j}^{\top}+\sum_{i \neq j} s_{j}^{2} s_{i}\left\langle g, a_{j}\right\rangle\left\langle a_{j}, a_{j}\right\rangle\left\langle a_{i}, a_{j}\right\rangle \cdot a_{i} a_{i}^{\top} \otimes a_{j} a_{j}^{\top} .
$$

By a union bound and an application of the triangle inequality it will be enough to show that just one of these two sums is $\tilde{O}\left(n^{3} / d^{4}\right)^{1 / 2}$ w.ov.p.. We rewrite the left-hand one:

$$
\sum_{i \neq j} s_{i}^{2} s_{j}\left\langle g, a_{i}\right\rangle\left\langle a_{i}, a_{i}\right\rangle\left\langle a_{i}, a_{j}\right\rangle \cdot a_{i} a_{i}^{\top} \otimes a_{j} a_{j}^{\top}=\sum_{j \in[n]} s_{j} a_{j} a_{j}^{\top} \otimes\left(\sum_{i \neq j}\left\langle g, a_{i}\right\rangle\left\|a_{i}\right\|^{2}\left\langle a_{i}, a_{j}\right\rangle \cdot a_{i} a_{i}^{\top}\right) .
$$

Define

$$
M_{j} \stackrel{\text { def }}{=} \sum_{i \neq j}\left\langle g, a_{i}\right\rangle\left\|a_{i}\right\|^{2}\left\langle a_{i}, a_{j}\right\rangle \cdot a_{i} a_{i}^{\top}
$$

so that now we need to bound $\sum_{j \in[n]} s_{j} a_{j} a_{j}^{\top} \otimes M_{j}$. By Corollary 5.15,

$$
\begin{aligned}
\left\|\sum_{j \in[n]} s_{j} a_{j} a_{j}^{\top} \otimes M_{j}\right\| & \stackrel{\text { w.ov.p. }}{\leqslant} \tilde{O}\left(\max _{j}\left\|M_{j}\right\|\right) \cdot \tilde{O}\left(\left\|\sum_{j \in[n]}\right\| a_{j}\left\|^{2} a_{j} a_{j}^{\top}\right\|^{1 / 2}\right) \\
& \leqslant \tilde{O}\left(\max _{j}\left\|M_{j}\right\|\right) \cdot \max _{j}\left\|a_{j}\right\| \cdot \tilde{O}\left(\left\|\sum_{j \in[n]} a_{j} a_{j}^{\top}\right\|^{1 / 2}\right)
\end{aligned}
$$

In Lemma 5.16, we bound $\max _{j}\left\|M_{j}\right\| \leqslant \tilde{O}\left(n / d^{2}\right)^{1 / 2}$ w.ov.p. using a matrix Bernstein inequality. Combining this bound with the concentration of $\left\|a_{j}\right\|$ around 1 (Fact 5.7), we obtain

$$
\begin{aligned}
& \stackrel{\text { w.ov.p. }}{\leqslant} \quad \tilde{O}\left(n / d^{2}\right)^{1 / 2} \cdot \tilde{O}(n / d)^{1 / 2} \\
& =\tilde{O}\left(n / d^{1.5}\right) .
\end{aligned}
$$

Having finished with $M_{\text {same, }}$ we turn to $M_{\text {diff. }}$

$$
\begin{aligned}
\left\|M_{\text {diff }}\right\| & =\left\|\sum_{\ell \neq i \neq j} s_{i} s_{j} s_{\ell}\left\langle g, a_{\ell}\right\rangle\left\langle a_{\ell}, a_{i}\right\rangle\left\langle a_{\ell}, a_{j}\right\rangle \cdot a_{i} a_{i}^{\top} \otimes a_{j} a_{j}^{\top}\right\| \\
& =\left\|\sum_{\ell} s_{\ell}\left\langle g, a_{\ell}\right\rangle\left(\sum_{i \neq \ell} s_{i}\left\langle a_{\ell}, a_{i}\right\rangle a_{i} a_{i}^{\top} \otimes\left(\sum_{j \neq \ell, i} s_{j}\left\langle a_{\ell}, a_{j}\right\rangle a_{j} a_{j}^{\top}\right)\right)\right\| .
\end{aligned}
$$

Letting $t_{1}, \ldots, t_{n}$ and $r_{1}, \ldots, r_{n}$ be independent uniformly random signs, by Theorem 5.13 , it will be enough to bound the spectral norm after replacing the second and third occurrences of $s_{i}$ for $t_{i}$ and $r_{i}$. To this end, we define

$$
M_{\text {diff }}^{\prime} \stackrel{\text { def }}{=} \sum_{\ell} s_{\ell}\left\langle g, a_{\ell}\right\rangle\left(\sum_{i \neq \ell} t_{i}\left\langle a_{\ell}, a_{i}\right\rangle a_{i} a_{i}^{\top} \otimes\left(\sum_{j \neq \ell, i} r_{j}\left\langle a_{\ell}, a_{j}\right\rangle a_{j} a_{j}^{\top}\right)\right) .
$$


Let

$$
N_{\ell} \stackrel{\text { def }}{=} \sum_{i \neq \ell} t_{i}\left\langle a_{\ell}, a_{i}\right\rangle a_{i} a_{i}^{\top} \otimes\left(\sum_{j \neq \ell, i} r_{j}\left\langle a_{\ell}, a_{j}\right\rangle a_{j} a_{j}^{\top}\right)
$$

so that we are to bound $\left\|\sum_{\ell \in[n]} s_{\ell}\left\langle g, a_{\ell}\right\rangle \cdot N_{\ell}\right\|$. By a matrix Rademacher bound and elementary manipulations,

$$
\begin{aligned}
& \left\|\sum_{\ell \in[n]} s_{\ell}\left\langle g, a_{\ell}\right\rangle \cdot N_{\ell}\right\| \stackrel{\text { w.ov.p. }}{\leqslant} \tilde{O}\left(\left\|\sum_{\ell \in[n]}\left\langle g, a_{\ell}\right\rangle^{2} \cdot N_{\ell}^{2}\right\|\right)^{1 / 2} \\
& \leqslant \tilde{O}(\sqrt{n}) \cdot \max _{\ell \in[n]}\left|\left\langle g, a_{\ell}\right\rangle\right| \cdot \max _{\ell \in[n]}\left\|N_{\ell}\right\| \\
& \stackrel{\text { w.ov.p. }}{\leqslant} \tilde{O}(\sqrt{n}) \cdot \max _{\ell \in[n]}\left\|N_{\ell}\right\| \quad \text { since }\left|\left\langle g, a_{i}\right\rangle\right| \leqslant \tilde{O}(1) \text { (Fact 5.7). }
\end{aligned}
$$

The rest of the proof is devoted to bounding $\left\|N_{\ell}\right\|$.

We start with Corollary 5.15 to get

$$
\left.\left\|N_{\ell}\right\| \stackrel{\text { w.ov.p. }}{\leqslant} \tilde{O}\left(\max _{i}\left\|\sum_{j \neq \ell, i} r_{j}\left\langle a_{\ell}, a_{j}\right\rangle \cdot a_{j} a_{j}^{\top}\right\|\right) \cdot\left\|\sum_{i \neq \ell}\left\langle a_{\ell}, a_{i}\right\rangle^{2}\right\| a_{i}\left\|^{2} \cdot a_{i} a_{i}^{\top}\right\|^{1 / 2}\right)
$$

We use a matrix Rademacher bound for the left-hand matrix,

$$
\begin{aligned}
\left\|\sum_{j \neq \ell, i} r_{j}\left\langle a_{\ell}, a_{j}\right\rangle \cdot a_{j} a_{j}^{\top}\right\| & \stackrel{\text { w.ov.p. }}{\leqslant} \tilde{O}\left(\left\|\sum_{j \neq \ell, i}\left\langle a_{\ell}, a_{j}\right\rangle^{2}\right\| a_{j}\left\|^{2} \cdot a_{j} a_{j}^{\top}\right\|\right)^{1 / 2} \\
& \leqslant \tilde{O}\left(\max _{j \neq \ell}\left\langle a_{\ell}, a_{j}\right\rangle^{2}\left\|a_{j}\right\|^{2}\left\|\sum_{j} a_{j} a_{j}^{\top}\right\|\right)^{1 / 2} \\
& \stackrel{\text { w.ov.p. }}{\leqslant} \tilde{O}\left(\frac{\sqrt{n}}{d}\right),
\end{aligned}
$$

where we have used that $\left\langle a_{\ell}, a_{i}\right\rangle^{2}$ concentrates around $\frac{1}{d}$ (Fact 5.7), that $\left\|a_{i}\right\|^{2}$ concentrates around 1 (Fact 5.7), and that $\left\|\sum_{i} a_{i} a_{i}^{\top}\right\|$ concentrates around $\frac{n}{d}$ (Lemma 5.16) within logarithmic factors all with overwhelming probability.

For the right-hand matrix, we use the fact that the summands are PSD to conclude that

$$
\begin{gathered}
\left\|\sum_{i \neq \ell}\left\langle a_{\ell}, a_{i}\right\rangle^{2}\right\| a_{i}\left\|^{2} \cdot a_{i} a_{i}^{\top}\right\| \\
\underset{\substack{\text { w.ov.p. } \\
i \neq \ell}}{\leqslant} \tilde{O}(1 / d) \cdot \tilde{O}(n / d),
\end{gathered}
$$

using the same concentration facts as earlier.

Putting these together, w.ov.p.

$$
\left\|N_{\ell}\right\| \leqslant \tilde{O}(\sqrt{n} / d) \cdot \tilde{O}(\sqrt{n} / d)=\tilde{O}\left(n / d^{2}\right) .
$$


Now we are ready to make the final bound on $M_{\text {diff }}^{\prime}$. With overwhelming probability,

$$
\left\|M_{\text {diff }}^{\prime}\right\| \leqslant \tilde{O}(\sqrt{n}) \cdot \max _{\ell \in[n]}\left\|N_{\ell}\right\| \leqslant \tilde{O}\left(n^{3} / d^{4}\right)^{1 / 2}
$$

and hence by Theorem 5.13, $\left\|M_{\text {diff }}\right\| \leqslant \tilde{O}(\sqrt{n}) \cdot \max _{\ell \in[n]}\left\|N_{\ell}\right\| \leqslant \tilde{O}\left(n^{3} / d^{4}\right)^{1 / 2}$ w.ov.p..

Finally, by triangle inequality and all our bounds thus far, w.ov.p.

$$
\left\|M_{\text {cross }}\right\| \leqslant\left\|M_{\text {same }}\right\|+\left\|M_{\text {diff }}\right\| \leqslant \tilde{O}\left(n / d^{1.5}\right)+\tilde{O}\left(n^{3} / d^{4}\right)^{1 / 2} \leqslant \tilde{O}\left(n^{3} / d^{4}\right)^{1 / 2} .
$$

\subsection{Full algorithm and proof of Theorem 5.2}

In this subsection we give the full details of our tensor decomposition algorithm. As discussed above, the algorithm proceeds by constructing a random matrix from the input tensor, then computing and post-processing its top eigenvector.

\section{Spectral Tensor Decomposition (One Attempt)}

This is the main subroutine of our algorithm-we will run it $\tilde{O}(n)$ times and show that this recovers all of the components $a_{1}, \ldots, a_{n}$.

Algorithm 5.17. Input: $\mathbf{T}=\sum_{i=1}^{n} a_{i} \otimes a_{i} \otimes a_{i}$. Goal: Recover $a_{i}$ for some $i \in[n]$.

- Compute the matrix unfolding $T \in \mathbb{R}^{d^{2} \times d}$ of $\mathbf{T}$. Then compute a 3-tensor $\mathbf{S} \in \mathbb{R}^{d^{2} \times d^{2} \times d^{2}}$ by starting with the 6-tensor $\mathbf{T} \otimes \mathbf{T}$, permuting indices, and flattening to a 3-tensor. Apply $T$ in one mode of $\mathbf{S}$ to obtain $\mathbf{M} \in \mathbb{R}^{d \otimes d^{2} \otimes d^{2}}$, so that:

$$
\begin{gathered}
T=\sum_{i \in[n]} a_{i}\left(a_{i} \otimes a_{i}\right)^{\top}, \quad \mathbf{S}=\mathbf{T}^{\otimes 2}=\sum_{i, j=1}^{n}\left(a_{i} \otimes a_{j}\right)^{\otimes 3}, \\
\mathbf{M}=\mathbf{S}\left(T, \operatorname{Id}_{d^{2}}, \mathrm{Id}_{d^{2}}\right)=\sum_{i, j \in[n]} T\left(a_{i} \otimes a_{j}\right) \otimes\left(a_{i} \otimes a_{j}\right) \otimes\left(a_{i} \otimes a_{j}\right) .
\end{gathered}
$$

- Sample a vector $g \in \mathbb{R}^{d}$ with iid standard gaussian entries. Evaluate $\mathbf{M}$ in its first mode in the direction of $g$ to obtain $M \in \mathbb{R}^{d^{2} \times d^{2}}$ :

$$
M:=\mathbf{M}\left(g, \operatorname{Id}_{d^{2}}, \operatorname{Id}_{d^{2}}\right)=\sum_{i, j \in[n]}\left\langle g, T\left(a_{i} \otimes a_{j}\right)\right\rangle \cdot\left(a_{i} \otimes a_{j}\right)\left(a_{i} \otimes a_{j}\right)^{\top}
$$

- Let $\Sigma \stackrel{\text { def }}{=} \mathbb{E}\left[\left(a a^{\top}\right)^{\otimes 2}\right]$ for $a \sim \mathcal{N}\left(0, \operatorname{Id}_{d}\right)$. Let $R \stackrel{\text { def }}{=} \sqrt{2} \cdot\left(\Sigma^{+}\right)^{1 / 2}$. Compute the top eigenvector $u \in \mathbb{R}^{d^{2}}$ of $R M R$, and reshape $R u$ to a matrix $U \in \mathbb{R}^{d \times d}$.

- For each of the signings of the top 2 unit left (or right) singular vectors $\pm u_{1}, \pm u_{2}$ of $U$, check if $\sum_{i \in[n]}\left\langle a_{i}, \pm u_{j}\right\rangle^{3} \geqslant 1-c(n, d)$ where $c(n, d)=\Theta\left(n / d^{3 / 2}\right)$ is an appropriate threshold. If so, output $\pm u_{j}$. Otherwise output nothing. 
Theorem 5.3 gets us most of the way to the correctness of Algorithm 5.17, proving that the top eigenvector of the matrix $R M R$ is correlated with some $a_{i}^{\otimes 2}$ with reasonable probability. We need a few more ingredients to prove Theorem 5.2. First, we need to show a bound on the runtime of Algorithm 5.17.

Lemma 5.18. Algorithm 5.17 can be implemented in time Õ $\left(d^{1+\omega}\right)$, where $d^{\omega}$ is the runtime for multiplying two $d \times d$ matrices. It may also be implemented in time $\tilde{O}\left(d^{3.257}\right)$.

Proof. To run the algorithm, we only require access to power iteration using the matrix $R M R$. We first give a fast implementation for power iteration with the matrix $M$, and handle the multiplications with $R$ separately.

Consider a vector $v \in \mathbb{R}^{d^{2}}$, and a random vector $g \sim \mathcal{N}\left(0, \operatorname{Id}_{d}\right)$, and let $V, G \in \mathbb{R}^{d \times d}$ be the reshapings of $v$ and $g T$ respectively into matrices. Call $\mathbf{T}_{v}=\mathbf{T}\left(\operatorname{Id}_{d}, V, G\right)$, where we have applied $V$ and $G$ in the second and third modes of $\mathbf{T}$, and call $T_{v}$ the reshaping of $\mathbf{T}_{v}$ into a $d \times d^{2}$ matrix. We have that

$$
T_{v}=\sum_{i \in[n]} a_{i}\left(V a_{i} \otimes G a_{i}\right)^{\top}
$$

We show that the matrix-vector multiply $M v$ can be computed as a flattening of the following product:

$$
\begin{aligned}
T_{v} T^{\top} & =\left(\sum_{i \in[n]} a_{i}\left(V a_{i} \otimes G a_{i}\right)^{\top}\right)\left(\sum_{j \in[n]}\left(a_{j} \otimes a_{j}\right) a_{j}^{\top}\right) \\
& =\sum_{i, j \in[n]}\left\langle a_{j}, V a_{i}\right\rangle \cdot\left\langle a_{j}, G a_{i}\right\rangle \cdot a_{i} a_{j}^{\top} \\
& =\sum_{i, j \in[n]}\left\langle a_{i} \otimes a_{j}, v\right\rangle \cdot\left\langle g T, a_{i} \otimes a_{j}\right\rangle \cdot a_{i} a_{j}^{\top}
\end{aligned}
$$

Flattening $T_{v} T^{\top}$ from a $d \times d$ matrix to a vector $v_{T T} \in \mathbb{R}^{d^{2}}$, we have that

$$
v_{T T}=\sum_{i, j \in[n]}\left\langle g T, a_{i} \otimes a_{j}\right\rangle \cdot\left\langle a_{i} \otimes a_{j}, v\right\rangle \cdot a_{i} \otimes a_{j}=M v .
$$

So we have that $M v$ is a flattening of the product $T_{v} T^{\top}$, which we will compute as a proxy for computing $M v$ via direct multiplication.

Computing $T_{v}=\mathbf{T}(\mathrm{Id}, V, G)$ can be done with two matrix multiplication operations, both times multiplying a $d^{2} \times d$ matrix with a $d \times d$ matrix. Computing $T_{v} T^{\top}$ is a multiplication of a $d \times d^{2}$ matrix by a $d^{2} \times d$ matrix. Both these steps may be done in time $O\left(d^{1+\omega}\right)$, by regarding the $d \times d^{2}$ matrices as block matrices with blocks of size $d \times d$. Alternatively, the asymptotically fastest known algorithm for rectangular matrix multiplication gives a time of $O\left(d^{3.257}\right)$ [LG12].

Now, to compute the matrix-vector multiply $R M R u$ for any vector $u \in \mathbb{R}^{d^{2}}$, we may first compute $v=R u$, perform the operation $M v$ in time $O\left(d^{1+\omega}\right)$ as described above, and then again multiply by $R$. The matrix $R$ is sparse: it has $O(d)$ entries per row (see Fact C.4), so the multiplication $R u$ requires time $O\left(d^{3}\right)$.

Performing the update $R M R v$ a total of $O\left(\log ^{2} n\right)$ times is sufficient for convergence, as we have that with reasonable probability, the spectral gap $\lambda_{2}(R M R) / \lambda_{1}(R M R) \leqslant 1-O\left(\frac{1}{\log n}\right)$, as a result of applying Theorem 5.3 with the choice of $\varepsilon=O\left(\frac{1}{\log n}\right)$. 
Finally, checking the value of $\sum_{i}\left\langle a_{i}, x\right\rangle^{3}$ requires $O\left(d^{3}\right)$ operations, and we do so a constant number of times, once for each of the signings of the top 2 left (or right) singular vectors of $U$.

Next, we need to show that given $u$ with $\left\langle R u, a_{i} \otimes a_{i}\right\rangle^{2} \geqslant\left(1-\tilde{O}\left(n^{3 / 2} / \varepsilon d^{2}\right)\right) \cdot\|u\|^{2} \cdot\left\|a_{i}\right\|^{4}$ we can actually recover the tensor component $a_{i}$. Here Algorithm 5.17 reshapes $R u$ to a $d \times d$ matrix and checks the top two left- or right-singular vectors; the next lemma shows one of these singular vectors must be highly correlated with $a_{i}$. (The proof is deferred to Section A.1.)

Lemma 5.19. Let $M \in \mathbb{R}^{d^{2} \times d^{2}}$ be a symmetric matrix with $\|M\| \leqslant 1$, and let $v \in \mathbb{R}^{d}$ and $u \in \mathbb{R}^{d^{2}}$ be vectors. Furthermore, let $U$ be the reshaping of the vector $M u \in \mathbb{R}^{d^{2}}$ to a matrix in $\mathbb{R}^{d \times d}$. Fix $c>0$, and suppose that $\langle M u, v \otimes v\rangle^{2} \geqslant c^{2} \cdot\|u\|^{2} \cdot\|v\|^{4}$. Then $U$ has some left singular vector a and some right singular vector $b$ such that

$$
|\langle a, v\rangle|,|\langle b, v\rangle| \geqslant c \cdot\|v\|
$$

Furthermore, for any $0<\alpha<1$, there are $a^{\prime}, b^{\prime}$ among the top $\left\lfloor\frac{1}{\alpha c^{2}}\right\rfloor$ singular vectors of $U$ with

$$
\left|\left\langle a^{\prime}, v\right\rangle\right|,\left|\left\langle b^{\prime}, v\right\rangle\right| \geqslant \sqrt{1-\alpha} \cdot c \cdot\|v\| .
$$

If $c \geqslant \sqrt{\frac{1}{2}(1+\eta)}$ for some $\eta>0$, then $a, b$ are amongst the top $\left\lfloor\frac{(1+\eta)}{\eta c^{2}}\right\rfloor$ singular vectors.

Since here $c^{2}=1-o(1)$, we can choose $\eta=1-o(1)$ and check only the top 2 singular vectors.

Next, we must show how to choose the threshold $c(n, d)$ so that a big enough value $\sum_{i \in[n]}\left\langle a_{i}, u_{j}\right\rangle^{3}$ is ensures that $u_{j}$ is close to a tensor component. The proof is at the end of this section. (A very similar fact appears in [GM15]. We need a somewhat different parameterization here, but we reuse many of their results in the proof.)

Lemma 5.20. Let $T=\sum_{i \in[n]} a_{i} \otimes a_{i} \otimes a_{i}$ for normally distributed vectors $a_{i} \sim \mathcal{N}\left(0, \frac{1}{d} \operatorname{Id}_{d}\right)$. For all $0<\gamma, \gamma^{\prime}<1$,

1. With overwhelming probability, for every $v \in \mathbb{R}^{d}$ such that $\sum_{i \in[n]}\left\langle a_{i}, v\right\rangle^{3} \geqslant 1-\gamma$,

$$
\max _{i \in[n]}\left|\left\langle a_{i}, v\right\rangle\right| \geqslant 1-O(\gamma)-\tilde{O}\left(n / d^{3 / 2}\right)
$$

2. With overwhelming probability over $a_{1}, \ldots, a_{n}$ if $v \in \mathbb{R}^{d}$ with $\|v\|=1$ satisfies $\left\langle v, a_{j}\right\rangle \geqslant 1-\gamma^{\prime}$ for some $j$ then $\sum_{i}\left\langle a_{i}, v\right\rangle^{3} \geqslant 1-O\left(\gamma^{\prime}\right)-\tilde{O}\left(n / d^{3 / 2}\right)$.

We are now ready to prove Theorem 5.2.

Proof of Theorem 5.2. By Theorem 5.3, with probability $1-o(1)$ over $a_{1}, \ldots, a_{n}$ there are events $E_{1}, \ldots, E_{n}$ so that $\mathbb{P}_{g}\left(E_{i}\right) \geqslant \tilde{O}\left(1 / n^{1+O(\varepsilon)}\right)$ such that when event $E_{i}$ occurs the top eigenvector $u$ of $R M R$ satisfies

$$
\frac{\left\langle R u, a_{i} \otimes a_{i}\right\rangle^{2}}{\|u\|^{2} \cdot\left\|a_{i}\right\|^{4}} \geqslant 1-\tilde{O}\left(\frac{n^{3 / 2}}{\varepsilon d^{2}}\right) .
$$

For a particular sample $g \sim \mathcal{N}\left(0, \operatorname{Id}_{d}\right)$, let $u_{g}$ be this eigenvector.

The algorithm is as follows. Sample $g_{1}, \ldots, g_{r} \sim \mathcal{N}\left(0, \mathrm{Id}_{d}\right)$ independently for some $r$ to be chosen later. Compute $R u_{g_{1}}, \ldots, R u_{g_{r}}$, reshape each to a $d \times d$ matrix, and compute its singular value decomposition. This gives a family of (right) singular vectors $v_{1}, \ldots, v_{d r}$. For each, evaluate 
$\sum_{i}\left\langle a_{i}, v_{j}\right\rangle^{3}$. Let $c(n, d)$ be a threshold to be chosen later. Initialize $S \subset \mathbb{R}^{d}$ to the empty set. Examining each $1 \leqslant j \leqslant d r$ in turn, add $v_{j}$ to $S$ if $\sum_{i}\left\langle a_{i}, v_{j}\right\rangle^{3} \geqslant 1-c(n, d)$ and for every $v$ already in $S,\left\langle v, v_{j}\right\rangle^{2} \leqslant 1 / 2$. Output the set $S$.

Choose $\varepsilon=1 / \log n$. By Lemma 5.19, when $E_{i}$ occurs for $g_{j}$ one of $v \in\left\{ \pm v_{j r}, \ldots, \pm v_{(j+1) r}\right\}$ has $\left\langle v, a_{i}\right\rangle \geqslant\left(1-\tilde{O}\left(n^{3 / 2} / d^{2}\right)\right)\left(\left\|u_{j}\right\|^{2} \cdot\left\|a_{j}\right\|^{4}\right)$. Then by Lemma 5.20 , when $E_{i}$ occurs for $g_{j}$, this $v$ we will have $\sum_{i}\left\langle a_{i}, \pm v\right\rangle^{3} \geqslant 1-\tilde{O}\left(n / d^{3 / 2}\right)$. Choose $c(n, d)=\tilde{\Theta}\left(n^{3 / 2} / d^{2}\right)$ so that when $E_{i}$ occurs for $g_{j}$, so long as it has not previously occurred for some $j^{\prime}<j$, the algorithm adds $\pm v$ to $S$.

The events $E_{i}^{(t)}$ and $E_{i}^{\left(t^{\prime}\right)}$ are independent for any two executions of the algorithm $t$ and $t^{\prime}$ and have probability $\tilde{\Omega}(1 / n)$. Thus, after $r=\tilde{O}(n)$ executions of the algorithm, with high probability for every $i \in[n]$ there is $j \in[r]$ so that $E_{i}$ occurs for $g_{j}$. Finally, by Lemma 5.20, the algorithm can never add to $S$ a vector which is not $\left(1-\tilde{O}\left(n / d^{3 / 2}\right)\right)$-close to some $a_{i}$.

It just remains to prove Lemma 5.20.

Proof of Lemma 5.20. We start with the first claim. By [GM15, Lemma 2, (proof of) Lemma 8, Theorem 4.2], the following inequalities all hold w.ov.p..

$$
\begin{aligned}
& \sum_{i \in n}\left\langle a_{i}, x\right\rangle^{4} \leqslant 1+\tilde{O}\left(n / d^{3 / 2}\right) \quad \text { for all }\|x\|=1, \\
& \sum_{i \in[n]}\left\langle a_{i}, x\right\rangle^{6} \geqslant 1-O\left(\sum_{i \in[n]}\left\langle a_{i}, x\right\rangle^{3}-1\right)-\tilde{O}\left(n / d^{3 / 2}\right) \quad \text { for all }\|x\|=1 \\
&\left|\sum_{i \in[n]}\left\langle a_{i}, x\right\rangle^{3}\right| \leqslant 1+\tilde{O}\left(n / d^{3 / 2}\right) \quad \text { for all }\|x\|=1 .
\end{aligned}
$$

To begin,

$$
\sum_{i \in[n]}\left\langle a_{i}, v\right\rangle^{6} \leqslant\left(\max _{i \in[n]}\left\langle a_{i}, v\right\rangle^{2}\right) \cdot\left(\sum_{i \in[n]}\left\langle a_{i}, v\right\rangle^{4}\right)
$$

By (5.6), this implies

$$
\max _{i \in[n]}\left\langle a_{i}, v\right\rangle^{2} \geqslant\left(1-\tilde{O}\left(n / d^{3 / 2}\right)\right) \cdot \sum_{i \in[n]}\left\langle v, a_{i}\right\rangle^{6} .
$$

Now combining (5.7) with (5.9) we have

$$
\max _{i \in[n]}\left\langle a_{i}, v\right\rangle^{2} \geqslant\left(1-\tilde{O}\left(n / d^{3 / 2}\right)\right) \cdot\left(1-O\left(1-\sum_{i}\left\langle a_{i}, v\right\rangle^{3}\right)-\tilde{O}\left(n / d^{3 / 2}\right)\right) .
$$

Together with (5.8) this concludes the of the first claim.

For the second claim, we note that by (5.8), and homogeneity, $\left|\sum_{i \neq j}\left\langle a_{i}, x\right\rangle^{3}\right| \leqslant\|x\|^{3}\left(1+\tilde{O}\left(n / d^{3 / 2}\right)\right.$ w.ov.p.. We write $v=\left\langle a_{j}, x\right\rangle a_{j}+x^{\perp}$, where $\left\langle x^{\perp}, a_{j}\right\rangle=0$. Now we expand

$$
\begin{aligned}
\sum_{i}\left\langle a_{i}, v\right\rangle^{3} & \geqslant\left(1-\gamma^{\prime}\right)^{3}+\sum_{i \neq j}\left\langle\left\langle a_{j}, x\right\rangle a_{j}+x^{\perp}, a_{i}\right\rangle^{3} \\
& =\left(1-\gamma^{\prime}\right)^{3}+\sum_{i \neq j}\left\langle a_{j}, x\right\rangle^{3}\left\langle a_{j}, a_{i}\right\rangle^{3}+3\left\langle a_{j}, x\right\rangle^{2}\left\langle a_{j}, a_{i}\right\rangle^{2}\left\langle x^{\perp}, a_{i}\right\rangle \\
& +3\left\langle a_{j}, x\right\rangle\left\langle a_{j}, a_{i}\right\rangle\left\langle x^{\perp} a_{i}\right\rangle^{2}+\left\langle x^{\perp}, a_{i}\right\rangle^{3} .
\end{aligned}
$$


We estimate each term in the expansion:

$$
\begin{aligned}
&\left|\sum_{i \neq j}\left\langle a_{j}, x\right\rangle^{3}\left\langle a_{j}, a_{i}\right\rangle^{3}\right| \leqslant\left|\left\langle a_{j}, x\right\rangle^{3}\right| \sum_{i \neq j}\left|\left\langle a_{j}, a_{i}\right\rangle\right|^{3} \leqslant \tilde{O}\left(\frac{n}{d^{3 / 2}}\right) \\
& \text { w.ov.p. by Cauchy-Schwarz and standard concentration. } \\
&\left|\sum_{i \neq j}\left\langle a_{j}, x\right\rangle^{2}\left\langle a_{j}, a_{i}\right\rangle^{2}\left\langle x^{\perp}, a_{i}\right\rangle\right| \leqslant\left(\sum_{i \neq j}\left\langle a_{j}, x\right\rangle^{4}\left\langle a_{j}, a_{i}\right\rangle^{4}\right)^{1 / 2}\left(\sum_{i \neq j}\left\langle x^{\perp}, a_{i}\right\rangle^{2}\right)^{1 / 2} \quad \text { by Cauchy-Schwarz } \\
& \leqslant O(\sqrt{n}) \cdot \max _{i \neq j}\left\langle a_{j}, a_{i}\right\rangle^{2} \cdot \tilde{O}\left(\frac{n}{d}\right)^{1 / 2} \quad \text { w.ov.p. by standard concentration. } \\
& \leqslant \tilde{O}\left(\frac{n}{d^{3 / 2}}\right) \quad \text { w.ov.p. by standard concentration } \\
&\left|\sum_{i \neq j}\left\langle a_{j}, x\right\rangle\left\langle a_{j}, a_{i}\right\rangle\left\langle x^{\perp}, a_{i}\right\rangle^{2}\right| \leqslant O(1) \cdot \max _{i \neq j}\left|\left\langle a_{j}, a_{i}\right\rangle\right|^{\cdot} \sum_{i \neq j}\left\langle x^{\perp}, a_{i}\right\rangle^{2} \quad \text { w.ov.p. by standard concentration } \\
& \leqslant \tilde{O}\left(\frac{1}{\sqrt{d}}\right) \cdot \tilde{O}\left(\frac{n}{d}\right) \quad \text { w.ov.p. by standard concentration } \\
& \leqslant \tilde{O}\left(\frac{n}{d^{3 / 2}}\right) \quad \leqslant \gamma^{\prime}+\tilde{O}\left(\frac{n}{d^{3 / 2}}\right) \quad \text { w.ov.p. by (5.8) and homogeneity . } \\
&\left|\sum_{i \neq j}\left\langle x^{\perp}, a_{i}\right\rangle^{3}\right|
\end{aligned}
$$

Now we estimate

$$
\sum_{i}\left\langle a_{i}, v\right\rangle^{3} \geqslant\left(1-\gamma^{\prime}\right)^{3}+\sum_{i \neq j}\left\langle a_{i}, x\right\rangle^{3} \geqslant(1-\gamma)^{3}-\gamma^{\prime}-\tilde{O}\left(n / d^{3 / 2}\right) \geqslant 1-O\left(\gamma^{\prime}\right)-\tilde{O}\left(n / d^{3 / 2}\right) .
$$

since $\gamma^{\prime}<1$.

\subsubsection{Boosting Accuracy with Local Search}

We remark that Algorithm 5.17 may be used in conjunction with a local search algorithm to obtain much greater guarantees on the accuracy of the recovered vectors. Previous progress on the tensor decomposition problem has produced iterative algorithms that provide local convergence guarantees given a good enough initialization, but which leave the question of how to initialize the procedure up to future work, or up to the specifics of an implementation. In this context, our contribution can be seen as a general method of obtaining good initializations for these local iterative procedures.

In particular, Anandkumar et al. [AGJ15] give an algorithm that combines tensor power iteration and a form of coordinate descent, which when initialized with the output of Algorithm 5.17, achieves a linear convergence rate to the true decomposition within polynomial time.

Theorem 5.21 (Adapted from Theorem 1 in [AGJ15]). Given a rank-n tensor $\mathbf{T}=\sum_{i} a_{i} \otimes a_{i} \otimes a_{i}$ with random Gaussian components $a_{i} \sim \mathcal{N}\left(0, \frac{1}{d} \mathrm{Id}_{d}\right)$. There is a constant $c>0$ so that if a set of unit vectors $\left\{x_{i} \in \mathbb{R}^{d}\right\}_{i}$ satisfies

$$
\left\langle x_{i}, a_{i}\right\rangle \geqslant 1-c, \quad \forall i \in[n]
$$


then there exists a procedure which with overwhelming probability over $\mathbf{T}$ and for any $\varepsilon>0$, recovers a set of vectors $\left\{\hat{a}_{i}\right\}$ such that

$$
\left\langle\hat{a}_{i}, a_{i}\right\rangle \geqslant 1-\varepsilon, \quad \forall i \in[n]
$$

in time $O\left(\operatorname{poly}(d)+n d^{3} \log \varepsilon\right)$.

Remark 5.22. Theorem 1 of Anandkumar et al. is stated for random asymmetric tensors, but the adaptation to symmetric tensors is stated in equations (14) and (27) in the same paper.

The theorem of Anandkumar et al. allows for a perturbation tensor $\Phi$, which is just the zero tensor in our setting. Additionally, the weight ratios specifying the weight of each rank-one component in the input tensor are $w_{\max }=w_{\min }=1$. Lastly, the initialization conditions are given in terms of the distance between the intialization vectors and the true vectors $\left|x_{i}-a_{i}\right|$, which is related to our measure of closeness $\left\langle x_{i}, a_{i}\right\rangle$ by the equation $\left|x_{i}-a_{i}\right|^{2}=\left|x_{i}\right|^{2}+\left|a_{i}\right|^{2}-2\left\langle x_{i}, a_{i}\right\rangle$.

The linear convergence guarantee is stated in Lemma 12 of Anandkumar et al.

Corollary 5.23 (Corollary of Theorem 5.2). Given as input the tensor $\mathbf{T}=\sum_{i=1}^{n} a_{i} \otimes a_{i} \otimes a_{i}$ where $a_{i} \sim \mathcal{N}\left(0, \frac{1}{d} \operatorname{Id}_{d}\right)$ with $d \leqslant n \leqslant d^{4 / 3} /$ polylog $d$, there is a polynomial-time algorithm which with probability $1-o(1)$ over the input $\mathbf{T}$ and the algorithm randomness finds unit vectors $\hat{a}_{1}, \ldots, \hat{a}_{n} \in \mathbb{R}^{d}$ such that for all $i \in[n]$,

$$
\left\langle\hat{a}_{i}, a_{i}\right\rangle \geqslant 1-O\left(2^{-n}\right) .
$$

Proof. We repeatedly invoke Algorithm 5.17 until we obtain a full set of $n$ vectors as characterized by Theorem 5.2. Apply Theorem 5.21 to the recovered set of vectors until the desired accuracy is obtained.

\section{Tensor principal component analysis}

The Tensor PCA problem in the spiked tensor model is similar to the setting of tensor decomposition, but here the goal is to recover a single large component with all smaller components of the tensor regarded as random noise.

Problem 6.1 (Tensor PCA in the Order-3 Spiked Tensor Model). Given an input tensor $\mathbf{T}=\tau \cdot v^{\otimes 3}+\mathbf{A}$, where $v \in \mathbb{R}^{n}$ is an arbitrary unit vector, $\tau \geqslant 0$ is the signal-to-noise ratio, and $\mathbf{A}$ is a random noise tensor with iid standard Gaussian entries, recover the signal $v$ approximately.

Using the partial trace method, we give the first linear-time algorithm for this problem that recovers $v$ for signal-to-noise ratio $\tau=O\left(n^{3 / 4} /\right.$ poly $\left.\log n\right)$. In addition, the algorithm requires only $O\left(n^{2}\right)$ auxiliary space (compared to the input size of $n^{3}$ ) and uses only one non-adaptive pass over the input.

\subsection{Spiked tensor model}

This spiked tensor model (for general order- $k$ tensors) was introduced by Montanari and Richard [RM14], who also obtained the first algorithms to solve the model with provable statistical guarantees. Subsequently, the SoS approach was applied to the model to improve the signal-to-noise ratio required for odd-order tensors [HSS15]; for 3-tensors reducing the requirement from $\tau=\Omega(n)$ to $\tau=\Omega\left(n^{3 / 4} \log (n)^{1 / 4}\right)$. 
Using the linear-algebraic objects involved in the analysis of the SoS relaxation, the previous work has also described algorithms with guarantees similar to those of the SoS SDP relaxation, while requiring only nearly subquadratic or linear time [HSS15].

The algorithm here improves on the previous results by use of the partial trace method, simplifying the analysis and improving the runtime by a factor of $\log n$.

\subsection{Linear-time algorithm}

\section{Linear-Time Algorithm for Tensor PCA}

Algorithm 6.2. Input: $\mathbf{T}=\tau \cdot v^{\otimes 3}+\mathbf{A}$. Goal: Recover $v^{\prime}$ with $\left\langle v, v^{\prime}\right\rangle \geqslant 1-o(1)$.

- Compute the partial trace $M:=\operatorname{Tr}_{\mathbb{R}^{n}} \sum_{i} T_{i} \otimes T_{i} \in \mathbb{R}^{n \times n}$, where $T_{i}$ are the first-mode slices of $\mathbf{T}$.

- Output the top eigenvector $v^{\prime}$ of $M$.

Theorem 6.3. When $\mathbf{A}$ has iid standard Gaussian entries and $\tau \geqslant C n^{3 / 4} \log (n)^{1 / 2} / \varepsilon$ for some constant $C$, Algorithm 6.2 recovers $v^{\prime}$ with $\left\langle v, v^{\prime}\right\rangle \geqslant 1-O(\varepsilon)$ with high probability over $\mathbf{A}$.

Theorem 6.4. Algorithm 6.2 can be implemented in linear time and sublinear space.

These theorems are proved by routine matrix concentration results, showing that in the partial trace matrix, the signal dominates the noise.

To implement the algorithm in linear time it is enough to show that this (sublinear-sized) matrix has constant spectral gap; then a standard application of the matrix power method computes the top eigenvector.

Lemma 6.5. For any $v$, with high probability over $\mathbf{A}$, the following occur:

$$
\begin{aligned}
\left\|\sum_{i} \operatorname{Tr}\left(A_{i}\right) \cdot A_{i}\right\| & \leqslant O\left(n^{3 / 2} \log ^{2} n\right) \\
\left\|\sum_{i} v(i) \cdot A_{i}\right\| & \leqslant O(\sqrt{n} \log n) \\
\left\|\sum_{i} \operatorname{Tr}\left(A_{i}\right) v(i) \cdot v v^{\top}\right\| & \leqslant O(\sqrt{n} \log n) .
\end{aligned}
$$

The proof may be found in Appendix D.

Proof of Theorem 6.3. We expand the partial trace $\operatorname{Tr}_{\mathbb{R}^{n}} \sum_{i} T_{i} \otimes T_{i}$.

$$
\begin{aligned}
\operatorname{Tr}_{\mathbb{R}^{n}} \sum_{i} T_{i} \otimes T_{i} & =\sum_{i} \operatorname{Tr}\left(T_{i}\right) \cdot T_{i} \\
& =\sum_{i} \operatorname{Tr}\left(\tau \cdot v(i) v v^{\top}+A_{i}\right) \cdot\left(\tau \cdot v(i) v v^{\top}+A_{i}\right) \\
& =\sum_{i}\left(\tau v(i)\|v\|^{2}+\operatorname{Tr}\left(A_{i}\right)\right) \cdot\left(\tau \cdot v(i) v v^{\top}+A_{i}\right)
\end{aligned}
$$




$$
=\tau^{2} v v^{\top}+\tau\left(\sum_{i} v(i) \cdot A_{i}+\sum_{i} \operatorname{Tr}\left(A_{i}\right) v(i) v v^{\top}\right)+\sum_{i} \operatorname{Tr}\left(A_{i}\right) \cdot A_{i} .
$$

Applying Lemma 6.5 and the triangle inequality, we see that

$$
\left\|\tau\left(\sum_{i} v(i) \cdot A_{i}+\sum_{i} \operatorname{Tr}\left(A_{i}\right) v(i) v v^{\top}\right)+\sum_{i} \operatorname{Tr}\left(A_{i}\right) \cdot A_{i}\right\| \leqslant O\left(n^{3 / 2} \log n\right)
$$

with high probability. Thus, for appropriate choice of $\tau=\Omega\left(n^{3 / 4} \sqrt{(\log n) / \varepsilon}\right)$, the matrix $\operatorname{Tr}_{\mathbb{R}^{n}} \sum_{i} T_{i} \otimes$ $T_{i}$ is close to rank one, and the result follows by standard manipulations.

Proof of Theorem 6.4. Carrying over the expansion of the partial trace from above and setting $\tau=$ $O\left(n^{3 / 4} \sqrt{(\log n) / \varepsilon}\right)$, the matrix $\operatorname{Tr}_{\mathbb{R}^{n}} \sum_{i} T_{i} \otimes T_{i}$ has a spectral gap ratio equal to $\Omega(1 / \varepsilon)$ and so the matrix power method finds the top eigenvector in $O(\log (n / \varepsilon))$ iterations. This matrix has dimension $n \times n$, so a single iteration takes $O\left(n^{2}\right)$ time, which is sublinear in the input size $n^{3}$. Finally, to construct $\operatorname{Tr}_{\mathbb{R}^{n}} \sum_{i} T_{i} \otimes T_{i}$ we use

$$
\operatorname{Tr}_{\mathbb{R}^{n}} \sum_{i} T_{i} \otimes T_{i}=\sum_{i} \operatorname{Tr}\left(T_{i}\right) \cdot T_{i}
$$

and note that to construct the right-hand side it is enough to examine each entry of $\mathbf{T}$ just $O(1)$ times and perform $O\left(n^{3}\right)$ additions. At no point do we need to store more than $O\left(n^{2}\right)$ matrix entries at the same time.

\section{Acknowledgements}

We would like to thank Rong Ge for very helpful discussions. We also thank Jonah Brown Cohen, Pasin Manurangsi and Aviad Rubinstein for helpful comments in the preparation of this manuscript.

\section{References}

[AFH ${ }^{+}$15] Anima Anandkumar, Dean P. Foster, Daniel Hsu, Sham M. Kakade, and Yi-Kai Liu, $A$ spectral algorithm for latent dirichlet allocation, Algorithmica 72 (2015), no. 1, 193-214. 3

[AGH $\left.{ }^{+} 14\right]$ Animashree Anandkumar, Rong Ge, Daniel Hsu, Sham M. Kakade, and Matus Telgarsky, Tensor decompositions for learning latent variable models, Journal of Machine Learning Research 15 (2014), no. 1, 2773-2832. 3

[AGHK14] Animashree Anandkumar, Rong Ge, Daniel Hsu, and Sham M. Kakade, A tensor approach to learning mixed membership community models, Journal of Machine Learning Research 15 (2014), no. 1, 2239-2312. 3

[AGJ14] Anima Anandkumar, Rong Ge, and Majid Janzamin, Analyzing tensor power method dynamics: Applications to learning overcomplete latent variable models, CoRR abs/1411.1488 (2014). 3 
[AGJ15] Animashree Anandkumar, Rong Ge, and Majid Janzamin, Learning overcomplete latent variable models through tensor methods, Proceedings of The 28th Conference on Learning Theory, COLT 2015, Paris, France, July 3-6, 2015, pp. 36-112. 3, 4, 20, 36

[AW02] Rudolf Ahlswede and Andreas J. Winter, Strong converse for identification via quantum channels, IEEE Transactions on Information Theory 48 (2002), no. 3, 569-579. 48

$\left[\mathrm{BBH}^{+} 12\right]$ Boaz Barak, Fernando G. S. L. Brandão, Aram Wettroth Harrow, Jonathan A. Kelner, David Steurer, and Yuan Zhou, Hypercontractivity, sum-of-squares proofs, and their applications, Proceedings of the 44th Symposium on Theory of Computing Conference, STOC 2012, New York, NY, USA, May 19 - 22, 2012, pp. 307-326. 1, 8

[BCMV14] Aditya Bhaskara, Moses Charikar, Ankur Moitra, and Aravindan Vijayaraghavan, Smoothed analysis of tensor decompositions, Symposium on Theory of Computing, STOC 2014, New York, NY, USA, May 31 - June 03, 2014, pp. 594-603. 3

[BKS14] Boaz Barak, Jonathan A. Kelner, and David Steurer, Rounding sum-of-squares relaxations, Symposium on Theory of Computing, STOC 2014, New York, NY, USA, May 31 - June 03, 2014, pp. 31-40. 1, 2, 3, 5, 6, 8, 43

[BKS15] _ Dictionary learning and tensor decomposition via the sum-of-squares method, Proceedings of the Forty-Seventh Annual ACM on Symposium on Theory of Computing, STOC 2015, Portland, OR, USA, June 14-17, 2015, pp. 143-151. 1, 3, 5, 8, 10, 11

[BM15] Boaz Barak and Ankur Moitra, Tensor prediction, rademacher complexity and random 3-xor, CoRR abs/1501.06521 (2015). 1, 6

[BRS11] Boaz Barak, Prasad Raghavendra, and David Steurer, Rounding semidefinite programming hierarchies via global correlation, IEEE 52nd Annual Symposium on Foundations of Computer Science, FOCS 2011, Palm Springs, CA, USA, October 22-25, 2011, pp. 472481. 6,8

[BS14] Boaz Barak and David Steurer, Sum-of-squares proofs and the quest toward optimal algorithms, CoRR abs/1404.5236 (2014). 1

[Cha96] J. T. Chang, Full reconstruction of markov models on evolutionary trees: Identifiability and consistency, Math Biosci. 137 (1996), 51-73. 3

[dGJL04] Alexandre d'Aspremont, Laurent El Ghaoui, Michael I. Jordan, and Gert R. G. Lanckriet, A direct formulation for sparse PCA using semidefinite programming, Advances in Neural Information Processing Systems 17 [Neural Information Processing Systems, NIPS 2004, December 13-18, Vancouver, British Columbia, Canada], 2004, pp. 41-48. 2

[DH14] Laurent Demanet and Paul Hand, Scaling law for recovering the sparsest element in a subspace, Information and Inference 3 (2014), no. 4, 295-309. 2, 3

[dlPMS95] Victor H de la Peña and Stephen J Montgomery-Smith, Decoupling inequalities for the tail probabilities of multivariate u-statistics, The Annals of Probability (1995), 806-816. 28 
[dlVK07] Wenceslas Fernandez de la Vega and Claire Kenyon-Mathieu, Linear programming relaxations of maxcut, Proceedings of the Eighteenth Annual ACM-SIAM Symposium on Discrete Algorithms, SODA 2007, New Orleans, Louisiana, USA, January 7-9, 2007, pp. 53-61. 6

[GHK15] Rong Ge, Qingqing Huang, and Sham M. Kakade, Learning mixtures of gaussians in high dimensions, Proceedings of the Forty-Seventh Annual ACM on Symposium on Theory of Computing, STOC 2015, Portland, OR, USA, June 14-17, 2015, pp. 761-770. 3

[GM15] Rong Ge and Tengyu Ma, Decomposing overcomplete 3rd order tensors using sum-of-squares algorithms, Approximation, Randomization, and Combinatorial Optimization. Algorithms and Techniques, APPROX/RANDOM 2015, August 24-26, Princeton, NJ, USA, 2015, pp. 829-849. 1, 3, 4, 5, 10, 11, 25, 28, 34, 35

[GS12] Venkatesan Guruswami and Ali Kemal Sinop, Faster SDP hierarchy solvers for local rounding algorithms, 53rd Annual IEEE Symposium on Foundations of Computer Science, FOCS 2012, New Brunswick, NJ, USA, October 20-23, 2012, pp. 197-206. 6

[GVX14] Navin Goyal, Santosh Vempala, and Ying Xiao, Fourier PCA and robust tensor decomposition, Symposium on Theory of Computing, STOC 2014, New York, NY, USA, May 31 - June 03, 2014, pp. 584-593. 3

[Har70] Richard A Harshman, Foundations of the PARAFAC procedure: Models and conditions for an "explanatory" multi-modal factor analysis, UCLA Working Papers in Phonetics 16 (1970), 1-84. 3

[Hås90] Johan Håstad, Tensor rank is np-complete, J. Algorithms 11 (1990), no. 4, 644-654. 3

[HL13] Christopher J. Hillar and Lek-Heng Lim, Most tensor problems are np-hard, J. ACM 60 (2013), no. 6, 45. 3

[HSS15] Samuel B. Hopkins, Jonathan Shi, and David Steurer, Tensor principal component analysis via sum-of-square proofs, Proceedings of The 28th Conference on Learning Theory, COLT 2015, Paris, France, July 3-6, 2015, pp. 956-1006. 1, 5, 6, 12, 37, 38

[Jan97] Svante Janson, Gaussian hilbert spaces, vol. 129, Cambridge university press, 1997. 46

[KB09] Tamara G. Kolda and Brett W. Bader, Tensor decompositions and applications, SIAM Review 51 (2009), no. 3, 455-500. 3

[Las01] Jean B. Lasserre, Global optimization with polynomials and the problem of moments, SIAM J. Optim. 11 (2000/01), no. 3, 796-817. MR 1814045 (2002b:90054) 1

[LCC07] Lieven De Lathauwer, Joséphine Castaing, and Jean-François Cardoso, Fourth-order cumulant-based blind identification of underdetermined mixtures, IEEE Transactions on Signal Processing 55 (2007), no. 6-2, 2965-2973. 3

[LG12] F. Le Gall, Faster algorithms for rectangular matrix multiplication, Foundations of Computer Science (FOCS), 2012 IEEE 53rd Annual Symposium on, Oct 2012, pp. 514-523. 33 
[LM00] B. Laurent and P. Massart, Adaptive estimation of a quadratic functional by model selection, Ann. Statist. 28 (2000), no. 5, 1302-1338. 52

[LR15] Elaine Levey and Thomas Rothvoss, A lasserre-based $(1+\varepsilon)$-approximation for $P m \mid p_{j}=$ 1, prec $\mid C_{\max }$, CoRR abs/1509.07808 (2015). 8

[MR06] Elchanan Mossel and Sébastien Roch, Learning nonsingular phylogenies and hidden markov models, Ann. Appl. Probab. 16 (2006), no. 2, 583-614. 3

[MW13] Raghu Meka and Avi Wigderson, Association schemes, non-commutative polynomial concentration, and sum-of-squares lower bounds for planted clique, CoRR abs/1307.7615 (2013). 8

[MW15] Tengyu Ma and Avi Wigderson, Sum-of-squares lower bounds for sparse PCA, CoRR abs/1507.06370 (2015). 6

[Nes00] Yurii Nesterov, Squared functional systems and optimization problems, High performance optimization, Appl. Optim., vol. 33, Kluwer Acad. Publ., Dordrecht, 2000, pp. 405-440. MR 1748764 (2001b:90063) 1

[NR09] Phong Q. Nguyen and Oded Regev, Learning a parallelepiped: Cryptanalysis of GGH and NTRU signatures, J. Cryptology 22 (2009), no. 2, 139-160. 3

[Par00] Pablo A Parrilo, Structured semidefinite programs and semialgebraic geometry methods in robustness and optimization, Ph.D. thesis, Citeseer, 2000. 1

[QSW14] Qing Qu, Ju Sun, and John Wright, Finding a sparse vector in a subspace: Linear sparsity using alternating directions, Advances in Neural Information Processing Systems 27: Annual Conference on Neural Information Processing Systems 2014, December 8-13 2014, Montreal, Quebec, Canada, 2014, pp. 3401-3409. 2, 3

[RM14] Emile Richard and Andrea Montanari, A statistical model for tensor PCA, Advances in Neural Information Processing Systems 27: Annual Conference on Neural Information Processing Systems 2014, December 8-13 2014, Montreal, Quebec, Canada, 2014, pp. 2897-2905. 4, 5, 12, 37

[RT12] Prasad Raghavendra and Ning Tan, Approximating csps with global cardinality constraints using SDP hierarchies, Proceedings of the Twenty-Third Annual ACM-SIAM Symposium on Discrete Algorithms, SODA 2012, Kyoto, Japan, January 17-19, 2012, pp. 373387. 8

[Sho87] N. Z. Shor, An approach to obtaining global extrema in polynomial problems of mathematical programming, Kibernetika (Kiev) (1987), no. 5, 102-106, 136. MR 931698 (89d:90202) 1

[SWW12] Daniel A. Spielman, Huan Wang, and John Wright, Exact recovery of sparsely-used dictionaries, COLT 2012 - The 25th Annual Conference on Learning Theory, June 25-27, Edinburgh, Scotland, 2012, pp. 37.1-37.18. 2 
[Tro12] Joel A. Tropp, User-friendly tail bounds for sums of random matrices, Foundations of Computational Mathematics 12 (2012), no. 4, 389-434. 28, 48

[Ver10] Roman Vershynin, Introduction to the non-asymptotic analysis of random matrices, CoRR abs/1011.3027 (2010). 51, 52, 53, 60

\section{A Additional preliminaries}

\section{A.1 Linear algebra}

Here we provide some lemmas in linear algebra.

This first lemma is closely related to the sos Cauchy-Schwarz from [BKS14], and the proof is essentially the same.

Lemma A.1 (PSD Cauchy-Schwarz). Let $M \in \mathbb{R}^{d \times d}, M \geq 0$ and symmetric. Let $p_{1}, \ldots, p_{n}, q_{1}, \ldots, q_{n} \in$ $\mathbb{R}^{d}$. Then

$$
\left\langle M, \sum_{i=1}^{n} p_{i} q_{i}^{\top}\right\rangle \leqslant\left\langle M, \sum_{i=1}^{n} p_{i} p_{i}^{\top}\right\rangle^{1 / 2}\left\langle M, \sum_{i=1}^{n} q_{i} q_{i}^{\top}\right\rangle^{1 / 2} .
$$

In applications, we will have $\sum_{i} p_{i} q_{i}$ as a single block of a larger block matrix containing also the blocks $\sum_{i} p_{i} p_{i}^{\top}$ and $\sum_{i} q_{i} q_{i}^{\top}$.

Proof. We first claim that

$$
\left\langle M, \sum_{i=1}^{n} p_{i} q_{i}^{\top}\right\rangle \leqslant \frac{1}{2}\left\langle M, \sum_{i=1}^{n} p_{i} p_{i}^{\top}\right\rangle+\frac{1}{2}\left\langle M, \sum_{i=1}^{n} q_{i} q_{i}^{\top}\right\rangle .
$$

To see this, just note that the right-hand side minus the left is exactly

$$
\left\langle M, \sum_{i=1}^{n}\left(p_{i}-q_{i}\right)\left(p_{i}-q_{i}\right)^{\top}\right\rangle=\sum_{i}\left(p_{i}-q_{i}\right)^{\top} M\left(p_{i}-q_{i}\right) \geqslant 0 .
$$

The lemma follows now be applying this inequality to

$$
p_{i}^{\prime}=\frac{p_{i}}{\left\langle M, \sum_{i=1}^{n} p_{i} p_{i}^{\top}\right\rangle^{1 / 2}} \quad q_{i}^{\prime}=\frac{q_{i}}{\left\langle M, \sum_{i=1}^{n} q_{i} q_{i}^{\top}\right\rangle^{1 / 2}} .
$$

Lemma A.2 (Operator Norm Cauchy-Schwarz for Sums). Let $A_{1}, \ldots, A_{m}, B_{1}, \ldots, B_{m}$ be real random matrices. Then

$$
\left\|\sum_{i} \mathbb{E} A_{i} B_{i}\right\| \leqslant\left\|\sum_{i} \mathbb{E} A_{i}^{\top} A_{i}\right\|^{1 / 2}\left\|\sum_{i} \mathbb{E} B_{i}^{\top} B_{i}\right\|^{1 / 2} .
$$

Proof. We have for any unit $x, y$,

$$
\begin{aligned}
x^{\top} \sum_{i} \mathbb{E} A_{i} B_{i} x & =\sum_{i} \mathbb{E}\left\langle A_{i} x, B_{i} y\right\rangle \\
& \leqslant \sum_{i} \mathbb{E}\left\|A_{i} x \mid\right\| B_{i} y \|
\end{aligned}
$$




$$
\begin{aligned}
& \leqslant \sum_{i}\left(\mathbb{E}\left\|A_{i} x\right\|^{2}\right)^{1 / 2}\left(\mathbb{E}\left\|B_{i} x\right\|^{2}\right)^{1 / 2} \\
& \leqslant \sqrt{\sum_{i} \mathbb{E}\left\|A_{i} x\right\|^{2}} \sqrt{\sum_{i} \mathbb{E}\left\|B_{i} y\right\|^{2}} \\
& =\sqrt{\mathbb{E} x^{\top} \sum_{i} A_{i}^{\top} A_{i} x} \sqrt{\mathbb{E} y^{\top} \sum_{i} B_{i}^{\top} B_{i} y} \\
& \leqslant\left\|\sum_{i} \mathbb{E} A_{i}^{\top} A_{i}\right\|^{1 / 2}\left\|\sum_{i} \mathbb{E} B_{i}^{\top} B_{i}\right\|^{1 / 2} .
\end{aligned}
$$

where the nontrivial inequalities follow from Cauchy-Schwarz for expectations, vectors and scalars, respectively.

The followng lemma allows to argue about the top eigenvector of matrices with spectral gap.

Lemma A.3 (Top eigenvector of gapped matrices). Let $M$ be a symmetric $r$-by-r matrix and let $u, v$ be a vectors in $\mathbb{R}^{r}$ with $\|u\|=1$. Suppose $u$ is a top singular vector of $M$ so that $|\langle u, M u\rangle|=\|M\|$ and $v$ satisfies for some $\varepsilon>0$,

$$
\left\|M-v v^{\top}\right\| \leqslant\|M\|-\varepsilon \cdot\|v\|^{2}
$$

Then, $\langle u, v\rangle^{2} \geqslant \varepsilon \cdot\|v\|^{2}$.

Proof. We lower bound the quadratic form of $M-v v^{\top}$ evaluated at $u$ by

$$
\left|\left\langle u,\left(M-v v^{\top}\right) u\right\rangle\right| \geqslant|\langle u, M u\rangle|-\langle u, v\rangle^{2}=\|M\|-\langle u, v\rangle^{2} .
$$

At the same time, this quadratic form evaluated at $u$ is upper bounded by $\|M\|-\varepsilon \cdot\|v\|^{2}$. It follows that $\langle u, v\rangle^{2} \geqslant \varepsilon \cdot\|v\|^{2}$ as desired.

The following lemma states that a vector in $\mathbb{R}^{d^{2}}$ which is close to a symmetric vector $v^{\otimes 2}$, if flattened to a matrix, has top eigenvector correlated with the symmetric vector.

Lemma (Restatement of Lemma 5.19). Let $M \in \mathbb{R}^{d^{2} \times d^{2}}$ be a symmetric matrix with $\|M\| \leqslant 1$, and let $v \in \mathbb{R}^{d}$ and $u \in \mathbb{R}^{d^{2}}$ be vectors. Furthermore, let $U$ be the reshaping of the vector $M u \in \mathbb{R}^{d^{2}}$ to a matrix in $\mathbb{R}^{d \times d}$. Fix $c>0$, and suppose that $\langle M u, v \otimes v\rangle^{2} \geqslant c^{2} \cdot\|u\|^{2} \cdot\|v\|^{4}$. Then $U$ has some left singular vector a and some right singular vector $b$ such that

$$
|\langle a, v\rangle|,|\langle b, v\rangle| \geqslant c \cdot\|v\| .
$$

Furthermore, for any $0<\alpha<1$, there are $a^{\prime}, b^{\prime}$ among the top $\left\lfloor\frac{1}{\alpha c^{2}}\right\rfloor$ singular vectors of $U$ with

$$
\left|\left\langle a^{\prime}, v\right\rangle\right|,\left|\left\langle b^{\prime}, v\right\rangle\right| \geqslant \sqrt{1-\alpha} \cdot c \cdot\|v\| .
$$

If $c \geqslant \sqrt{\frac{1}{2}(1+\eta)}$ for some $\eta>0$, then $a, b$ are amongst the top $\left\lfloor\frac{(1+\eta)}{\eta c^{2}}\right\rfloor$ singular vectors. 
Proof. Let $\hat{v}=v /\|v\|$. Let $\left(\sigma_{i}, a_{i}, b_{i}\right)$ be the $i$ th singular value, left and right (unit) singular vectors of $U$ respectively.

Our assumptions imply that

$$
\left|\hat{v}^{\top} U \hat{v}\right|=|\langle M u, \hat{v} \otimes \hat{v}\rangle| \geqslant c \cdot\|u\| .
$$

Furthermore, we observe that $\|U\|_{F}=\|M u\| \leqslant\|M\| \cdot\|u\|$, and that therefore $\|U\|_{F} \leqslant\|u\|$. We thus have that,

$$
c \cdot\|u\| \leqslant\left|\hat{v}^{\top} U \hat{v}\right|=\left|\sum_{i \in[d]} \sigma_{i} \cdot\left\langle\hat{v}, a_{i}\right\rangle\left\langle\hat{v}, b_{i}\right\rangle\right| \leqslant\|u\| \cdot \sqrt{\sum_{i \in[d]}\left\langle\hat{v}, a_{i}\right\rangle^{2}\left\langle\hat{v}, b_{i}\right\rangle^{2}},
$$

where to obtain the last inequality we have used Cauchy-Schwarz and our bound on $\|U\|_{F}$. We may thus conclude that

$$
c^{2} \leqslant \sum_{i \in[d]}\left\langle\hat{v}, a_{i}\right\rangle^{2}\left\langle\hat{v}, b_{i}\right\rangle^{2} \leqslant \max _{i \in[d]}\left\langle a_{i}, \hat{v}\right\rangle^{2} \cdot \sum_{i \in[d]}\left\langle b_{i}, \hat{v}\right\rangle^{2}=\max _{i \in[d]}\left\langle a_{i}, \hat{v}\right\rangle^{2},
$$

where we have used the fact that the left singular values of $U$ are orthonormal. The argument is symmetric in the $b_{i}$.

Furthermore, we have that

$$
c^{2} \cdot\|u\|^{2} \leqslant\left|\hat{v}^{\top} U \hat{v}\right|^{2}=\left|\sum_{i \in[d]} \sigma_{i} \cdot\left\langle\hat{v}, a_{i}\right\rangle\left\langle\hat{v}, b_{i}\right\rangle\right|^{2} \leqslant\left(\sum_{i \in[d]} \sigma_{i}^{2}\left\langle\hat{v}, a_{i}\right\rangle^{2}\right) \cdot\left(\sum_{i \in[d]}\left\langle\hat{v}, b_{i}\right\rangle^{2}\right)=\sum_{i \in[d]} \sigma_{i}^{2}\left\langle\hat{v}, a_{i}\right\rangle^{2},
$$

where we have applied Cauchy-Schwarz and the orthonormality of the $b_{i}$. In particular,

$$
\sum_{i \in[d]} \sigma_{i}^{2}\left\langle\hat{v}, a_{i}\right\rangle^{2} \geqslant c^{2}\|u\|^{2} \geqslant c^{2}\|U\|_{F}^{2} .
$$

On the other hand, let $S$ be the set of $i \in[d]$ for which $\sigma_{i}^{2} \leqslant \alpha c^{2}\|U\|_{F}^{2}$. By substitution,

$$
\sum_{i \in S} \sigma_{i}^{2}\left\langle\hat{v}, a_{i}\right\rangle^{2} \leqslant \alpha c^{2}\|U\|_{F}^{2} \sum_{i \in S}\left\langle\hat{v}, a_{i}\right\rangle^{2} \leqslant \alpha c^{2}\|U\|_{F}^{2}
$$

where we have used the fact that the right singular vectors are orthonormal. The last two inequalities imply that $S \neq[d]$. Letting $T=[d] \backslash S$, it follows from subtraction that

$$
(1-\alpha) c^{2}\|U\|_{F}^{2} \leqslant \sum_{i \in T} \sigma_{i}^{2}\left\langle\hat{v}, a_{i}\right\rangle^{2} \leqslant \max _{i \in T}\left\langle\hat{v}, a_{i}\right\rangle^{2} \sum_{i \in T} \sigma_{i}^{2}=\max _{i \in T}\left\langle\hat{v}, a_{i}\right\rangle^{2}\|U\|_{F}^{2},
$$

so that $\max _{i \in T}\left\langle\hat{v}, a_{i}\right\rangle^{2} \geqslant(1-\alpha) c^{2}$. Finally,

$$
|T| \cdot \alpha c^{2}\|U\|_{F}^{2} \leqslant|T| \cdot \min _{i \in T} \sigma_{i}^{2} \leqslant \sum_{i \in[d]} \sigma_{i}^{2}=\|U\|_{F}^{2}
$$

so that $|T| \leqslant\left\lfloor\frac{1}{\alpha c^{2}}\right\rfloor$. Thus, one of the top $\left\lfloor\frac{1}{\alpha c^{2}}\right\rfloor$ right singular vectors $a$ has correlation $|\langle\hat{v}, a\rangle| \geqslant$ $\sqrt{(1-\alpha)} c$. The same proof holds for the $b$.

Furthermore, if $c^{2}>\frac{1}{2}(1+\eta)$ for some $\eta>0$, and $(1-\alpha) c^{2}>\frac{1}{2}$, then by (A.1) it must be that $\max _{i \in T}\left\langle\hat{v}, a_{i}\right\rangle^{2}=\max _{i \in[d]}\left\langle\hat{v}, a_{i}\right\rangle^{2}$, as $\hat{v}$ cannot have square correlation larger than $\frac{1}{2}$ with more than one left singular vector. Taking $\alpha=\frac{\eta}{1+\eta}$ guarantees this. The conclusion follows. 


\section{A.2 Concentration tools}

We require a number of tools from the literature on concentration of measure.

\section{A.2.1 For scalar-valued polynomials of Gaussians}

We need the some concentration bounds for certain polynomials of Gaussian random variables.

The following lemma gives standard bounds on the tails of a standard gaussian variablesomewhat more precisely than other bounds in this paper. Though there are ample sources, we repeat the proof here for reference.

Lemma A.4. Let $X \sim \mathcal{N}(0,1)$. Then for $t>0$,

$$
\mathbb{P}(X>t) \leqslant \frac{e^{-t^{2} / 2}}{t \sqrt{2 \pi}}
$$

and

$$
\mathbb{P}(X>t) \geqslant \frac{e^{-t^{2} / 2}}{\sqrt{2 \pi}} \cdot\left(\frac{1}{t}-\frac{1}{t^{3}}\right) .
$$

Proof. To show the first statement, we apply an integration trick,

$$
\begin{aligned}
\mathbb{P}(X>t) & =\frac{1}{\sqrt{2 \pi}} \int_{t}^{\infty} e^{-x^{2} / 2} d x \\
& \leqslant \frac{1}{\sqrt{2 \pi}} \int_{t}^{\infty} \frac{x}{t} e^{-x^{2} / 2} d x \\
& =\frac{e^{-t^{2} / 2}}{t \sqrt{2 \pi}}
\end{aligned}
$$

where in the third step we have used the fact that $\frac{x}{t} \leqslant x$ for $t \geqslant x$. For the second statement, we integrate by parts and repeat the trick,

$$
\begin{aligned}
\mathbb{P}(X>t) & =\frac{1}{\sqrt{2 \pi}} \int_{t}^{\infty} e^{-x^{2} / 2} d x \\
& =\frac{1}{\sqrt{2 \pi}} \int_{t}^{\infty} \frac{1}{x} \cdot x e^{-x^{2} / 2} d x \\
& =\frac{1}{\sqrt{2 \pi}}\left[-\frac{1}{x} e^{-x^{2} / 2} \cdot\right]_{t}^{\infty}-\frac{1}{\sqrt{2 \pi}} \int_{t}^{\infty} \frac{1}{x^{2}} \cdot e^{-x^{2} / 2} d x \\
& \geqslant \frac{1}{\sqrt{2 \pi}}\left[-\frac{1}{x} e^{-x^{2} / 2} \cdot\right]_{t}^{\infty}-\frac{1}{\sqrt{2 \pi}} \int_{t}^{\infty} \frac{x}{t^{3}} \cdot e^{-x^{2} / 2} d x \\
& =\frac{1}{\sqrt{2 \pi}}\left(\frac{1}{t}-\frac{1}{t^{3}}\right) e^{-t^{2} / 2} .
\end{aligned}
$$

This concludes the proof.

The following is a small modification of Theorem 6.7 from [Jan97] which follows from Remark 6.8 in the same. 
Lemma A.5. For each $\ell \geqslant 1$ there is a universal constant $c_{\ell}>0$ such that for every $f$ a degree- $\ell$ polynomial of standard Gaussian random variables $X_{1}, \ldots, X_{m}$ and $t \geqslant 2$,

$$
\mathbb{P}(|f(X)|>t \mathbb{E}|f(X)|) \leqslant e^{-c_{\ell} t^{2 / \ell}} .
$$

The same holds (with a different constant $c_{\ell}$ ) if $\mathbb{E}|f(x)|$ is replaced by $\left(\mathbb{E} f(x)^{2}\right)^{1 / 2}$.

In our concentration results, we will need to calculate the expectations of multivariate Gaussian polynomials, many of which share a common form. Below we give an expression for these expectations.

Fact A.6. Let $x$ be a d-dimensional vector with independent identically distributed gaussian entries with variance $\sigma^{2}$. Let $u$ be a fixed unit vector. Then setting $X=\left(\|x\|^{2}-c\right)^{p}\|x\|^{2 m} x x^{T}$, and setting $U=$ $\left(\|x\|^{2}-c\right)^{p}\|x\|^{2 m} u u^{T}$, we have

$$
\mathbb{E}[X]=\left(\sum_{0 \leqslant k \leqslant p}\left(\begin{array}{l}
p \\
k
\end{array}\right)(-1)^{k} c^{k}(d+2) \cdots(d+2 p+2 m-2 k) \sigma^{2(p+m-k+1)}\right) \cdot \mathrm{Id},
$$

and

$$
\mathbb{E}[U]=\left(\sum_{0 \leqslant k \leqslant p}\left(\begin{array}{l}
p \\
k
\end{array}\right)(-1)^{k} c^{k} d(d+2) \cdots(d+2 p+2 m-2 k-2) \sigma^{2(p+m-k)}\right) \cdot u u^{T}
$$

Proof.

$$
\begin{aligned}
\mathbb{E}[X] & =\mathbb{E}\left[\left(\|x\|^{2}-c\right)^{p}\|x\|^{2 m} x_{1}^{2}\right] \cdot \mathrm{Id} \\
& =\mathrm{Id} \cdot \sum_{0 \leqslant k \leqslant p}\left(\begin{array}{l}
p \\
k
\end{array}\right)(-1)^{k} c^{k} \mathbb{E}\left[\left(\sum_{\ell \in[d]} x_{i}^{2}\right)^{p+m-k} x_{1}^{2}\right]
\end{aligned}
$$

Since $\left(\sum_{i \in[d]} x_{i}^{2}\right)^{p+m-k}$ is symmetric in $x_{1}, \ldots, x_{d}$, we have

$$
=\operatorname{Id} \cdot \frac{1}{d} \sum_{0 \leqslant k \leqslant p}\left(\begin{array}{l}
p \\
k
\end{array}\right)(-1)^{k} c^{k} \mathbb{E}\left[\left(\sum_{i \in[d]} x_{i}^{2}\right)^{p+m-k+1}\right]
$$

We have reduced the computation to a question of the moments of a Chi-squared variable with $d$ degrees of freedom. Using these moments,

$$
\begin{aligned}
& =\operatorname{Id} \cdot \frac{1}{d} \sum_{0 \leqslant k \leqslant p}\left(\begin{array}{l}
p \\
k
\end{array}\right)(-1)^{k} c^{k} d(d+2) \cdots(d+2 p+2 m-2 k) \sigma^{2(p+m-k+1)} \\
& =\operatorname{Id} \cdot\left(\sum_{0 \leqslant k \leqslant p}\left(\begin{array}{l}
p \\
k
\end{array}\right)(-1)^{k} c^{k}(d+2) \cdots(d+2 p+2 m-2 k) \sigma^{2(p+m-k+1)}\right) .
\end{aligned}
$$

A similar computation yields the result about $\mathbb{E}[U]$. 


\section{A.2.2 For matrix-valued random variables}

On several occasions we will need to apply a Matrix-Bernstein-like theorem to a sum of matrices with an unfortunate tail. To this end, we prove a "truncated Matrix Bernstein Inequality." Our proof uses an standard matrix Bernstein inequality as a black box. The study of inequalities of this variety - on tails of sums of independent matrix-valued random variables - was initiated by Ahlswede and Winter [AW02]. The excellent survey of Tropp [Tro12] provides many results of this kind.

In applications of the following the operator norms of the summands $X_{1}, \ldots, X_{n}$ have wellbehaved tails and so the truncation is a routine formality. Two corollaries following the proposition and its proof capture truncation for all the matrices we encounter in the present work.

Proposition A.7 (Truncated Matrix Bernstein). Let $X_{1}, \ldots, X_{n} \in \mathbb{R}^{d_{1} \times d_{2}}$ be independent random matrices, and suppose that

$$
\mathbb{P}\left[\left\|X_{i}-\mathbb{E}\left[X_{i}\right]\right\|_{o p} \geqslant \beta\right] \leqslant p \text { for all } i \in[n] .
$$

Furthermore, suppose that for each $X_{i}$,

$$
\left\|\mathbb{E}\left[X_{i}\right]-\mathbb{E}\left[X_{i} \mathbb{I}\left[\left\|X_{i}\right\|_{o p}<\beta\right]\right]\right\| \leqslant q .
$$

Denote

$$
\sigma^{2}=\max \left\{\left\|\sum_{i \in[n]} \mathbb{E}\left[X_{i} X_{i}^{T}\right]-\mathbb{E}\left[X_{i}\right] \mathbb{E}\left[X_{i}^{T}\right]\right\|_{o p},\left\|\sum_{i \in[n]} \mathbb{E}\left[X_{i}^{T} X_{i}\right]-\mathbb{E}\left[X_{i}\right]^{T} \mathbb{E}\left[X_{i}\right]\right\|_{o p}\right\} .
$$

Then for $X=\sum_{i \in[n]} X_{i}$, we have

$$
\mathbb{P}\left[\|X-\mathbb{E}[X]\|_{o p} \geqslant t\right] \leqslant n \cdot p+\left(d_{1}+d_{2}\right) \cdot \exp \left(\frac{-(t-n q)^{2}}{2\left(\sigma^{2}+\beta(t-n q) / 3\right)}\right) .
$$

Proof. For simplicity we start by centering the variables $X_{i}$. Let $\tilde{X}_{i}=X_{i}-\mathbb{E} X_{i}$ and $\tilde{X}=\sum_{i \in[n]} \tilde{X}_{i}$ The proof proceeds by a straightforward application of the noncommutative Bernstein's Inequality. We define variables $Y_{1}, \ldots, Y_{n}$, which are the truncated counterparts of the $\tilde{X}_{i}$ s in the following sense:

$$
Y_{i}= \begin{cases}\tilde{X}_{i} & \left\|\tilde{X}_{i}\right\|_{o p}<\beta \\ 0 & \text { otherwise }\end{cases}
$$

Define $Y=\sum_{i \in[n]} Y_{i}$. We claim that

$$
\begin{aligned}
& \left\|\sum_{i} \mathbb{E} Y_{i} Y_{i}^{T}-\mathbb{E}\left[Y_{i}\right] \mathbb{E}\left[Y_{i}\right]^{T}\right\|_{\mathrm{op}} \leqslant\left\|\sum_{i} \mathbb{E} \tilde{X}_{i} \tilde{X}_{i}^{T}\right\|_{\mathrm{op}} \leqslant \sigma^{2} \text { and } \\
& \left\|\sum_{i} \mathbb{E} Y_{i}^{T} Y_{i}-\mathbb{E}\left[Y_{i}\right]^{T} \mathbb{E}\left[Y_{i}\right]\right\|_{\mathrm{op}} \leqslant\left\|\sum_{i} \mathbb{E} \tilde{X}_{i}^{T} \tilde{X}_{i}\right\|_{\mathrm{op}} \leqslant \sigma^{2},
\end{aligned}
$$

which, together with the fact that $\left\|Y_{i}\right\| \leqslant \beta$ almost surely, will allow us to apply the noncommutative Bernstein's inequality to $Y$. To see (A.2) ((A.3) is similar), we expand $\mathbb{E} Y_{i} Y_{i}^{T}$ as

$$
\mathbb{E} Y_{i} Y_{i}^{T}=\mathbb{P}\left[\left\|\tilde{X}_{i}\right\|_{\mathrm{op}}<\beta\right] \mathbb{E}\left[\tilde{X}_{i} \tilde{X}_{i}^{T} \mid\left\|\tilde{X}_{i}\right\|_{\mathrm{op}}<\beta\right] .
$$


Additionally expanding $\mathbb{E}\left[\tilde{X}_{i} \tilde{X}_{i}^{T}\right]$ as

$$
\mathbb{E}\left[\tilde{X}_{i} \tilde{X}_{i}^{T}\right]=\mathbb{P}\left[\left\|\tilde{X}_{i}\right\|_{\mathrm{op}}<\beta\right] \mathbb{E}\left[\tilde{X}_{i} \tilde{X}_{i}^{T} \mid\left\|\tilde{X}_{i}\right\|_{\mathrm{op}}<\beta\right]+\mathbb{P}\left[\left\|\tilde{X}_{i}\right\|_{\mathrm{op}} \geqslant \beta\right] \mathbb{E}\left[\tilde{X}_{i} \tilde{X}_{i}^{T} \mid\left\|\tilde{X}_{i}\right\|_{\mathrm{op}} \geqslant \beta\right],
$$

we note that $\mathbb{E}\left[\tilde{X}_{i} \tilde{X}_{i}^{T} \mid\left\|\tilde{X}_{i}\right\|_{\text {op }} \geqslant \beta\right]$ is PSD. Thus, $\mathbb{E}\left[Y_{i} Y_{i}^{T}\right] \geq \mathbb{E}\left[X_{i} X_{i}^{T}\right]$. But by definition $\mathbb{E}\left[Y_{i} Y_{i}^{T}\right]$ is still PSD (and hence $\left\|\sum_{i} \mathbb{E}\left[Y_{i} Y_{i}^{T}\right]\right\|_{\text {op }}$ is given by the maximum eigenvalue of $\mathbb{E}\left[Y_{i} Y_{i}^{T}\right]$ ), so

$$
\left\|\sum_{i} \mathbb{E} Y_{i} Y_{i}^{T}\right\|_{\mathrm{op}} \leqslant\left\|\sum_{i} \mathbb{E} \tilde{X}_{i} \tilde{X}_{i}^{T}\right\|_{\mathrm{op}} .
$$

Also PSD are $\mathbb{E}\left[Y_{i}\right] \mathbb{E}\left[Y_{i}\right]^{T}$ and $\mathbb{E}\left[\left(Y_{i}-\mathbb{E}\left[Y_{i}\right]\right)\left(Y_{i}-\mathbb{E}\left[Y_{i}\right]\right)^{T}\right]=\mathbb{E}\left[Y_{i} Y_{i}^{T}\right]-\mathbb{E}\left[Y_{i}\right] \mathbb{E}\left[Y_{i}\right]^{T}$. By the same reasoning again, then, we get $\left\|\sum_{i} \mathbb{E} Y_{i} Y_{i}^{T}-\mathbb{E}\left[Y_{i}\right] \mathbb{E}\left[Y_{i}\right]^{T}\right\|_{\mathrm{op}} \leqslant\left\|\sum_{i} \mathbb{E}\left[Y_{i} Y_{i}^{T}\right]\right\|_{\mathrm{op}}$. Putting this all together gives (A.2).

Now we are ready to apply the non-commutative Bernstein's inequality to $Y$. We have

$$
\mathbb{P}\left[\|Y-\mathbb{E}[Y]\|_{o p} \geqslant \alpha\right] \leqslant\left(d_{1}+d_{2}\right) \cdot \exp \left(\frac{-\alpha^{2} / 2}{\sigma^{2}+\beta \cdot \alpha / 3}\right) .
$$

Now, we have

$$
\begin{aligned}
\mathbb{P}\left[\|X-\mathbb{E}[X]\|_{o p} \geqslant t\right] & =\mathbb{P}\left[\|X-\mathbb{E}[X]\|_{o p} \geqslant t \mid X=Y\right] \cdot \mathbb{P}[X=Y] \\
+ & \mathbb{P}\left[\|X-\mathbb{E}[X]\|_{o p} \geqslant t \mid X \neq Y\right] \cdot \mathbb{P}[X \neq Y] \\
& \leqslant \mathbb{P}\left[\|X-\mathbb{E}[X]\|_{o p} \geqslant t \mid X=Y\right]+n \cdot p
\end{aligned}
$$

by a union bound over the events $\left\{X_{i} \neq Y_{i}\right\}$. It remains to bound the conditional probability $\mathbb{P}\left[\|X-\mathbb{E}[X]\|_{o p} \geqslant t \mid X=Y\right]$. By assumption, $\|\mathbb{E}[X]-\mathbb{E}[Y]\|_{o p} \leqslant n q$, and so by the triangle inequality,

$$
\|X-\mathbb{E}[X]\|_{o p} \leqslant\|X-\mathbb{E}[Y]\|_{o p}+\|\mathbb{E}[Y]-\mathbb{E}[X]\|_{o p} \leqslant\|X-\mathbb{E}[Y]\|_{o p}+n q .
$$

Thus,

$$
\begin{aligned}
\mathbb{P}\left[\|X-\mathbb{E}[X]\|_{o p} \geqslant t \mid X=Y\right] & \leqslant \mathbb{P}\left[\|X-\mathbb{E}[Y]\|_{o p}+n q \geqslant t \mid X=Y\right] \\
& =\mathbb{P}\left[\|Y-\mathbb{E}[Y]\|_{o p} \geqslant t-n q \mid X=Y\right] .
\end{aligned}
$$

Putting everything together and setting $\alpha=t-n q$,

$$
\mathbb{P}\left[\|X-\mathbb{E}[X]\|_{o p} \geqslant t\right] \leqslant n \cdot p+\left(d_{1}+d_{2}\right) \cdot \exp \left(\frac{-(t-n q)^{2} / 2}{\sigma^{2}+\beta(t-n q) / 3}\right),
$$

as desired.

The following lemma helps achieve the assumptions of Proposition A.7 easily for a useful class of thin-tailed random matrices. 
Lemma A.8. Suppose that $X$ is a matrix whose entries are polynomials of constant degree $\ell$ in unknowns $x$, which we evaluate at independent Gaussians. Let $f(x):=\|X\|_{o p}$ and $g(x):=\left\|X X^{T}\right\|_{o p}$, and either $f$ is itself a polynomial in $x$ of degree at most $2 \ell$ or $g$ is a polynomial in $x$ of degree at most $4 \ell$. Then if $\beta=R \cdot \alpha$ for $\alpha \geqslant \min \{\mathbb{E}[|f(x)|], \sqrt{\mathbb{E}[g(x)]}\}$ and $R=\operatorname{polylog}(n)$,

$$
\mathbb{P}\left(\|X\|_{o p} \geqslant \beta\right) \leqslant n^{-\log n},
$$

and

$$
\mathbb{E}\left[\left\|X \cdot \mathbb{I}\left\{\|X\|_{o p} \geqslant \beta\right\}\right\|_{o p}\right] \leqslant(\beta+\alpha) n^{-\log n} .
$$

Proof. We begin with (A.4). Either $f(x)$ is a polynomial of degree at most $2 \ell$, or $g(x)$ is a polynomial of degree at most $4 \ell$ in gaussian variables. We can thus use Lemma A.5 to obtain the following bound,

$$
\mathbb{P}(|f(x)| \geqslant t \alpha) \leqslant \exp \left(-c t^{1 /(2 \ell)}\right),
$$

where $c$ is a universal constant. Taking $t=R=\operatorname{polylog}(n)$ gives us (A.4).

We now address (A.5). To this end, let $p(t)$ and $P(t)$ be the probability density function and cumulative density function of $\|X\|_{o p}$, respectively. We apply Jensen's inequality and instead bound

$$
\left\|\mathbb{E}\left[X \mathbb{I}\left\{\|X\|_{o p} \geqslant \beta\right\}\right]\right\| \leqslant \mathbb{E}\left[\|X\|_{o p} \mathbb{I}\left\{\|X\|_{o p} \geqslant \beta\right\}\right]=\int_{0}^{\infty} t \cdot \mathbb{I}\{t \geqslant \beta\} p(t) d t
$$

since the indicator is 0 for $t \leqslant \beta$,

$$
=\int_{\beta}^{\infty}(-t)(-p(t)) d t
$$

integrating by parts,

$$
=-\left.t \cdot(1-P(t))\right|_{\beta} ^{\infty}+\int_{\beta}^{\infty}(1-P(t)) d t
$$

and using the equality of $1-P(t)$ with $\mathbb{P}\left(\|X\|_{o p} \geqslant t\right)$ along with (A.4),

$$
\leqslant \beta n^{-\log n}+\int_{\beta}^{\infty} \mathbb{P}\left(\|X\|_{o p} \geqslant t\right) d t
$$

Applying the change of variables $t=\alpha s$ so as to apply (A.6),

$$
\begin{aligned}
& =\beta n^{-\log n}+\alpha \int_{R}^{\infty} \mathbb{P}\left(\|X\|_{o p} \geqslant \alpha s\right) d s \\
& \leqslant \beta n^{-\log n}+\alpha \int_{R}^{\infty} \exp \left(-c s^{1 /(2 \ell)}\right) d s
\end{aligned}
$$


Now applying a change of variables so $s=\left(\frac{u \log n}{c}\right)^{2 \ell}$,

$$
\begin{aligned}
& =\beta n^{-\log n}+\alpha \int_{\frac{c R^{1 /(2 \ell)}}{\log n}}^{\infty} n^{-u} \cdot 2 \ell\left(\frac{\log n}{c}\right)^{2 \ell} u^{2 \ell-1} d u \\
& \leqslant \beta n^{-\log n}+\alpha \int_{\frac{c R^{1 /(2 \ell)}}{\log n}}^{\infty} n^{-u / 2} d u,
\end{aligned}
$$

where we have used the assumption that $\ell$ is constant. We can approximate this by a geometric sum,

$$
\begin{aligned}
& \leqslant \beta n^{-\log n}+\alpha \sum_{u=\frac{\left.c R^{1 / 2 \ell}\right)}{\log n}}^{\infty} n^{-u / 2} \\
& \leqslant \beta n^{-\log n}+\alpha \cdot n^{-c R^{1 /(2 \ell)} /(2 \log n)}
\end{aligned}
$$

Evaluating at $R=$ polylog $n$ for a sufficiently large polynomial in the log gives us

$$
\mathbb{E}\left[\left\|X \cdot \mathbb{I}\left\{\|X\|_{o p} \geqslant \beta\right\}\right\|_{o p}\right] \leqslant(\beta+\alpha) n^{-\log n},
$$

as desired.

\section{B Concentration bounds for planted sparse vector in random linear subspace}

Proof of Lemma 4.7. Let $c:=\sum_{i=1}^{n} v(i) b_{i}$. The matrix in question has a nice block structure:

$$
\sum_{i=1}^{n} a_{i} a_{i}^{\top}=\left(\begin{array}{cc}
\|v\|_{2}^{2} & c^{\top} \\
c & \sum_{i=1}^{n} b_{i} b_{i}^{\top}
\end{array}\right) .
$$

The vector $c$ is distributed as $\mathcal{N}\left(0, \frac{1}{n} \operatorname{Id}_{d-1}\right)$ so by standard concentration has $\|c\| \leqslant \tilde{O}(d / n)^{1 / 2}$ w.ov.p.. By assumption, $\|v\|_{2}^{2}=1$. Thus by triangle inequality w.ov.p.

$$
\left\|\sum_{i=1}^{n} a_{i} a_{i}^{\top}-\mathrm{Id}_{d}\right\| \leqslant \tilde{O}\left(\frac{d}{n}\right)^{1 / 2}+\left\|\sum_{i=1}^{n} b_{i} b_{i}^{\top}-\mathrm{Id}_{d-1}\right\| .
$$

By [Ver10, Corollary 5.50] applied to the subgaussian vectors $n b_{i}$, w.ov.p.

$$
\left\|\sum_{i=1}^{n} b_{i} b_{i}^{\top}-\mathrm{Id}_{d-1}\right\| \leqslant O\left(\frac{d}{n}\right)^{1 / 2}
$$

and hence $\left\|\sum_{i=1}^{n} a_{i} a_{i}^{\top}-\operatorname{Id}_{d}\right\| \leqslant \tilde{O}(d / n)^{1 / 2}$ w.ov.p.. This implies $\left\|\left(\sum_{i=1}^{n} a_{i} a_{i}^{\top}\right)^{-1}-\operatorname{Id}_{d}\right\| \leqslant \tilde{O}(d / n)^{1 / 2}$ and $\left\|\left(\sum_{i=1}^{n} a_{i} a_{i}^{\top}\right)^{-1 / 2}-\operatorname{Id}_{d}\right\| \leqslant \tilde{O}(d / n)^{1 / 2}$ when $d=o(n)$ by the following facts applied to the eigenvalues of $\sum_{i=1}^{n} a_{i} a_{i}^{\top}$. For $0 \leqslant \varepsilon<1$,

$$
\begin{aligned}
(1+\varepsilon)^{-1} & =1-O(\varepsilon) & \text { and } & (1-\varepsilon)^{-1}=1+O(\varepsilon), \\
(1+\varepsilon)^{-1 / 2} & =1-O(\varepsilon) & \text { and } & (1-\varepsilon)^{-1 / 2}=1+O(\varepsilon) .
\end{aligned}
$$

These are proved easily via the identity $(1+\varepsilon)^{-1}=\sum_{k=1}^{\infty} \varepsilon^{k}$ and similar. 


\section{Orthogonal subspace basis}

Lemma B.1. Let $a_{1}, \ldots, a_{n} \in \mathbb{R}^{d}$ be independent random vectors from $N\left(0, \frac{1}{n} \mathrm{Id}\right)$ with $d \leqslant n$ and let $A=\sum_{i=1}^{n} a_{i} a_{i}^{\top}$. Then for every unit vector $x \in \mathbb{R}^{d}$, with overwhelming probability $1-d^{-\omega(1)}$,

$$
\left|\left\langle x, A^{-1} x\right\rangle-\|x\|^{2}\right| \leqslant \tilde{O}\left(\frac{d+\sqrt{n}}{n}\right) \cdot\|x\|^{2} .
$$

Proof. Let $x \in \mathbb{R}^{d}$. By scale invariance, we may assume $\|x\|=1$.

By standard matrix concentration bounds, the matrix $B=\mathrm{Id}-A$ has spectral norm $\|B\| \leqslant$ $\tilde{O}(d / n)^{1 / 2}$ w.ov.p. [Ver10, Corollary 5.50]. Since $A^{-1}=(\operatorname{Id}-B)^{-1}=\sum_{k=0}^{\infty} B^{k}$, the spectral norm of $A^{-1}-\mathrm{Id}-B$ is at most $\sum_{k=2}^{\infty}\|B\|^{k}$ (whenever the series converges). Hence, $\left\|A^{-1}-\mathrm{Id}-B\right\| \leqslant \tilde{O}(d / n)$ w.ov.p..

It follows that it is enough to show that $|\langle x, B x\rangle| \leqslant \tilde{O}(1 / n)^{1 / 2}$ w.ov.p.. The random variable $n-n\langle x, B x\rangle=\sum_{i=1}^{n}\left\langle\sqrt{n} \cdot a_{i}, x\right\rangle^{2}$ is $\chi^{2}$-distributed with $n$ degrees of freedom. Thus, by standard concentration bounds, $n|\langle x, B x\rangle| \leqslant \tilde{O}(\sqrt{n})$ w.ov.p. [LM00].

We conclude that with overwhelming probability $1-d^{-\omega(1)}$,

$$
\left|\left\langle x, A^{-1} x\right\rangle-\|x\|^{2}\right| \leqslant|\langle x, B x\rangle|+\tilde{O}(d / n) \leqslant \tilde{O}\left(\frac{d+\sqrt{n}}{n}\right) .
$$

Lemma B.2. Let $a_{1}, \ldots, a_{n} \in \mathbb{R}^{d}$ be independent random vectors from $N\left(0, \frac{1}{n} \mathrm{Id}\right)$ with $d \leqslant n$ and let $A=\sum_{i=1}^{n} a_{i} a_{i}^{\top}$. Then for every index $i \in[n]$, with overwhelming probability $1-d^{\omega(1)}$,

$$
\left|\left\langle a_{j}, A^{-1} a_{j}\right\rangle-\left\|a_{j}\right\|^{2}\right| \leqslant \tilde{O}\left(\frac{d+\sqrt{n}}{n}\right) \cdot\left\|a_{j}\right\|^{2} .
$$

Proof. Let $A_{-j}=\sum_{i \neq j} a_{i} a_{i}^{\top}$. By Sherman-Morrison,

$$
A^{-1}=\left(A_{-j}+a_{j} a_{j}^{\top}\right)^{-1}=A_{-j}^{-1}-\frac{1}{1+a_{j}^{\top} A_{-j}^{-1} a_{j}} A_{-j}^{-1} a_{j} a_{j}^{\top} A_{-j}^{-1}
$$

Thus, $\left\langle a_{j}, A^{-1} a_{j}\right\rangle=\left\langle a_{j}, A_{-j}^{-1} a_{j}\right\rangle-\left\langle a_{j}, A_{-j}^{-1} a_{j}\right\rangle^{2} /\left(1+\left\langle a_{j}, A_{-j}^{-1} a_{j}\right\rangle\right)$. Since $\left\|\frac{n}{n-1} A_{-j}-\operatorname{Id}\right\|=\tilde{O}(d / n)^{1 / 2}$ w.ov.p., we also have $\left\|A_{-j}^{-1}\right\| \leqslant 2$ with overwhelming probability. Therefore, w.ov.p.,

$$
\left|\left\langle a_{j}, A^{-1} a_{j}\right\rangle-\left\langle a_{j}, A_{-j}^{-1} a_{j}\right\rangle\right| \leqslant\left\langle a_{j}, A_{-j}^{-1} a_{j}\right\rangle^{2} \leqslant 4\left\|a_{j}\right\|^{4} \leqslant \tilde{O}(d / n) \cdot\left\|a_{j}\right\|^{2} .
$$

At the same time, by Lemma B.1, w.ov.p.,

$$
\left|\left\langle a_{j}, \frac{n}{n-1} A_{-j}^{-1} a_{j}\right\rangle-\left\|a_{j}\right\|^{2}\right| \leqslant \tilde{O}\left(\frac{d+\sqrt{n}}{n}\right) \cdot\left\|a_{j}\right\|^{2} .
$$

We conclude that, w.ov.p.,

$$
\begin{aligned}
\left|\left\langle a_{j}, A^{-1} a_{j}\right\rangle-\left\|a_{j}\right\|^{2}\right| & \leqslant\left|\left\langle a_{j}, A^{-1} a_{j}\right\rangle-\left\langle a_{j}, A_{-j}^{-1} a_{j}\right\rangle\right|+\left|\left\langle a_{j}, A_{-j}^{-1} a_{j}\right\rangle-\frac{n-1}{n}\left\|a_{j}\right\|^{2}\right|+\frac{1}{n}\left\|a_{j}\right\|^{2} \\
& \leqslant \tilde{O}\left(\frac{d+\sqrt{n}}{n}\right) .
\end{aligned}
$$


Lemma B.3. Let $A$ be a block matrix where one of the diagonal blocks is the $1 \times 1$ identity; that is,

$$
A=\left(\begin{array}{cc}
\|v\|^{2} & c^{\top} \\
c & B
\end{array}\right)=\left(\begin{array}{cc}
1 & c^{\top} \\
c & B
\end{array}\right)
$$

for some matrix $B$ and vector $c$. Let $x$ be a vector which decomposes as $x=\left(x(1) x^{\prime}\right)$ where $x(1)=\left\langle x, e_{1}\right\rangle$ for $e_{1}$ the first standard basis vector.

Then

$$
\left\langle x, A^{-1} x\right\rangle=\left\langle x^{\prime},\left(B^{-1}+\frac{B^{-1} c c^{\top} B^{-1}}{1-c^{\top} B^{-1} c}\right) x^{\prime}\right\rangle+2 x(1)\left\langle\left(B^{-1}+\frac{B^{-1} c c^{\top} B^{-1}}{1-c^{\top} B^{-1} c}\right) c, x^{\prime}\right\rangle+\left(1-c^{\top} B^{-1} c\right)^{-1} x(1)^{2} .
$$

Proof. By the formula for block matrix inverses,

$$
A^{-1}=\left(\begin{array}{cc}
\left(1-c^{\top} B^{-1} c\right)^{-1} & c^{T}\left(B-c C^{\top}\right)^{-1} \\
\left(B-c C^{\top}\right)^{-1} c & \left(B-c C^{\top}\right)^{-1}
\end{array}\right) .
$$

The result follows by Sherman-Morrison applied to $\left(B-c C^{\top}\right)^{-1}$ and the definition of $x$.

Lemma B.4. Let $v \in \mathbb{R}^{n}$ be a unit vector and let $b_{1}, \ldots, b_{n} \in \mathbb{R}^{d-1}$ have iid entries from $\mathcal{N}(0,1 / n)$. Let $a_{i} \in \mathbb{R}^{d}$ be given by $a_{i}:=\left(v(i) b_{i}\right)$. Let $A:=\sum_{i} a_{i} a_{i}^{T}$. Let $c \in \mathbb{R}^{d-1}$ be given by $c:=\sum_{i} v(i) b_{i}$. Then for every index $i \in[n]$,w.ov.p.,

$$
\left|\left\langle a_{i}, A^{-1} a_{i}\right\rangle-\left\|a_{i}\right\|^{2}\right| \leqslant \tilde{O}\left(\frac{d+\sqrt{n}}{n}\right) \cdot\left\|a_{i}\right\|^{2} .
$$

Proof. Let $B:=\sum_{i} b_{i} b_{i}^{T}$. By standard concentration, $\left\|B^{-1}-\mathrm{Id}\right\| \leqslant \tilde{O}(d / n)^{1 / 2}$ w.ov.p. [Ver10, Corollary 5.50]. At the same time, since $v$ has unit norm, the entries of $c$ are iid samples from $\mathcal{N}(0,1 / n)$, and hence $n\|c\|^{2}$ is $\chi^{2}$-distributed with $d$ degrees of freedom. Thus w.ov.p. $\|c\|^{2} \leqslant \frac{d}{n}+\tilde{O}(d n)^{-1 / 2}$. Together these imply the following useful estimates, all of which hold w.ov.p.:

$$
\begin{aligned}
\left|c^{\top} B^{-1} c\right| & \leqslant\|c\|^{2}\left\|B^{-1}\right\|_{o p} \leqslant \frac{d}{n}+\tilde{O}\left(\frac{d}{n}\right)^{3 / 2} \\
\left\|B^{-1} c c^{\top} B^{-1}\right\|_{o p} & \leqslant\|c\|^{2}\left\|B^{-1}\right\|_{o p}^{2} \leqslant \frac{d}{n}+\tilde{O}\left(\frac{d}{n}\right)^{3 / 2} \\
\left\|\frac{B^{-1} c c^{\top} B^{-1}}{1-c^{\top} B^{-1} c}\right\|_{o p} & \leqslant \frac{d}{n}+\tilde{O}\left(\frac{d}{n}\right)^{3 / 2},
\end{aligned}
$$

where the first two use Cauchy-Schwarz and the last follows from the first two.

We turn now to the expansion of $\left\langle a_{i}, A^{-1} a_{i}\right\rangle$ offered by Lemma B.3,

$$
\begin{aligned}
\left\langle a_{i}, A^{-1} a_{i}\right\rangle= & \left\langle b_{i},\left(B^{-1}+\frac{B^{-1} c c^{\top} B^{-1}}{1-c^{\top} B^{-1} c}\right) b_{i}\right\rangle \\
& +2 v(i)\left\langle\left(B^{-1}+\frac{B^{-1} c c^{\top} B^{-1}}{1-c^{\top} B^{-1} c}\right) c, b_{i}\right\rangle \\
& +\left(1-c^{\top} B^{-1} c\right)^{-1} v(i)^{2} .
\end{aligned}
$$


Addressing (B.1) first, by the above estimates and Lemma B.2 applied to $\left\langle b_{i}, B^{-1} b_{i}\right\rangle$,

$$
\left|\left\langle b_{i},\left(B^{-1}+\frac{B^{-1} c c^{\top} B^{-1}}{1-c^{\top} B^{-1} c}\right) b_{i}\right\rangle-\left\|b_{i}\right\|^{2}\right| \leqslant \tilde{O}\left(\frac{d+\sqrt{n}}{n}\right) \cdot\left\|b_{i}\right\|^{2}
$$

w.ov.p.. For (B.2), we pull out the important factor of $\|c\|$ and separate $v(i)$ from $b_{i}$ : w.ov.p.,

$$
\begin{aligned}
\left|2 v(i)\left\langle\left(B^{-1}+\frac{B^{-1} c c^{\top} B^{-1}}{1-c^{\top} B^{-1} c}\right) c, b_{i}\right\rangle\right| & =\left|2\|c\| v(i)\left\langle\left(B^{-1}+\frac{B^{-1} c c^{\top} B^{-1}}{1-c^{\top} B^{-1} c}\right) \frac{c}{\|c\|}, b_{i}\right\rangle\right| \\
& \leqslant\left|\|c\|^{2}\left(v(i)^{2}+\left\langle\left(B^{-1}+\frac{B^{-1} c c^{\top} B^{-1}}{1-c^{\top} B^{-1} c}\right) \frac{c}{\|c\|^{\prime}}, b_{i}\right\rangle^{2}\right)\right| \\
& \leqslant \tilde{O}\left(\frac{d}{n}\right)\left(v(i)^{2}+\left\|b_{i}\right\|^{2}\right) \\
& =\tilde{O}\left(\frac{d}{n}\right)\left\|a_{i}\right\|^{2},
\end{aligned}
$$

where the last inequality follows from our estimates above and Cauchy-Schwarz.

Finally, for (B.3), since $\left(1-c^{\top} B^{-1} c\right) \geqslant 1-\tilde{O}(d / n)$ w.ov.p., we have that

$$
\left|\left(1-c^{\top} B^{-1} c\right)^{-1} v(i)^{2}-v(i)^{2}\right| \leqslant \tilde{O}\left(\frac{d}{n}\right) v(i)^{2}
$$

Putting it all together,

$$
\begin{aligned}
\left|\left\langle a_{i}, A^{-1} a_{i}\right\rangle-\left\|a_{i}\right\|^{2}\right| & \leqslant\left|\left\langle b_{i},\left(B^{-1}+\frac{B^{-1} c c^{\top} B^{-1}}{1-c^{\top} B^{-1} c}\right) b_{i}\right\rangle-\left\|b_{i}\right\|^{2}\right| \\
& +\left|2 v(i)\left\langle\left(B^{-1}+\frac{B^{-1} c c^{\top} B^{-1}}{1-c^{\top} B^{-1} c}\right) c, b_{i}\right\rangle\right| \\
& +\left|\left(1-c^{\top} B^{-1} c\right)^{-1} v(i)^{2}-v(i)^{2}\right| \\
& \leqslant \tilde{O}\left(\frac{d+\sqrt{n}}{n}\right) \cdot\left\|a_{i}\right\|^{2}
\end{aligned}
$$

\section{Concentration bounds for overcomplete tensor decomposition}

We require some facts about the concentration of certain scalar-and matrix-valued random variables, which generally follow from standard concentration arguments. We present proofs here for completeness.

The first lemma captures standard facts about random Gaussians.

Fact C.1. Let $a_{1}, \ldots, a_{n} \in \mathbb{R}^{d}$ be sampled $a_{i} \sim \mathcal{N}\left(0, \frac{1}{d} \mathrm{Id}\right)$.

1. Inner products $\left|\left\langle a_{i}, a_{j}\right\rangle\right|$ are all $\approx 1 / \sqrt{d}$ :

$$
\mathbb{P}\left\{\left\langle a_{i}, a_{j}\right\rangle^{2} \leqslant \tilde{O}\left(\frac{1}{d}\right) \mid \forall i, j \in[n], i \neq j\right\} \geqslant 1-n^{-\omega(1)} .
$$


2. Norms are all about $\left\|a_{i}\right\| \approx 1 \pm \tilde{O}(1 / \sqrt{d})$ :

$$
\mathbb{P}\left\{1-\tilde{O}(1 / \sqrt{d}) \leqslant\left\|a_{i}\right\|_{2}^{2} \leqslant 1+\tilde{O}(1 / \sqrt{d}) \mid \forall i \in[n]\right\} \geqslant 1-n^{-\omega(1)} .
$$

3. Fix a vector $v \in \mathbb{R}^{d}$. Suppose $g \in \mathbb{R}^{d}$ is a vector with entries identically distributed $g_{i} \sim \mathcal{N}(0, \sigma)$. Then $\langle g, v\rangle^{2} \approx \sigma^{2} \cdot\|v\|_{2}^{2}$ :

$$
\mathbb{P}\left\{\left|\langle g, v\rangle^{2}-\sigma^{2} \cdot\|v\|_{2}^{4}\right| \leqslant \tilde{O}\left(\sigma^{2} \cdot\|v\|_{4}^{2}\right)\right\} \geqslant 1-n^{-\omega(1)} .
$$

Proof of Fact C.1. We start with Item 1. Consider the quantity $\left\langle a_{i}, a_{j}\right\rangle^{2}$. We calculate the expectation,

$$
\mathbb{E}\left[\left\langle a_{i}, a_{j}\right\rangle^{2}\right]=\sum_{k, \ell \in[d]} \mathbb{E}\left[a_{i}(k) a_{i}(\ell) a_{j}(k) a_{j}(\ell)\right]=\sum_{k \in[d]} \mathbb{E}\left[a_{i}(k)^{2}\right] \cdot \mathbb{E}\left[a_{j}(k)^{2}\right]=d \cdot \frac{1}{d^{2}}=\frac{1}{d} .
$$

Since this is a degree- 4 square polynomial in the entries of $a_{i}$ and $a_{j}$, we may apply Lemma A.5 to conclude that

$$
\mathbb{P}\left(\left\langle a_{i}, a_{j}\right\rangle^{2} \geqslant t \cdot \frac{1}{d}\right) \leqslant \exp \left(-O\left(t^{1 / 2}\right)\right)
$$

Applying this fact with $t=\operatorname{polylog}(n)$ and taking a union bound over pairs $i, j \in[n]$ gives us the desired result.

Next is Item 2. Consider the quantity $\left\|a_{i}\right\|_{2}^{2}$. We will apply Lemma A.5 in order to obtain a tail bound for the value of the polynomial $\left(\left\|a_{i}\right\|_{2}^{2}-1\right)^{2}$. We have

$$
\mathbb{E}\left[\left(\left\|a_{i}\right\|_{2}^{2}-1\right)^{2}\right]=O\left(\frac{1}{d}\right),
$$

and now applying Lemma A.5 with the square root of this expectation, we have

$$
\mathbb{P}\left(||\left|a_{i} \|_{2}^{2}-1\right| \geqslant \tilde{O}\left(\frac{1}{\sqrt{d}}\right)\right) \leqslant n^{-\log n} .
$$

This gives both bounds for a single $a_{i}$. The result now follows from taking a union bound over all $i$.

Moving on to Item 3, we view the expression $f(g):=\left(\langle g, v\rangle^{2}-\sigma^{2}\|v\|^{2}\right)^{2}$ as a polynomial in the gaussian entries of $g$. The degree of $f(g)$ is 4 , and $\mathbb{E}[|f(g)|]=3 \sigma^{4} \cdot\|v\|_{4}^{4}$, and so we may apply Lemma A.5 to conclude that

$$
\mathbb{P}\left(|f(g)| \geqslant t \cdot 3 \sigma^{4} \cdot\|v\|_{4}^{4}\right) \leqslant \exp \left(-c_{4} t^{1 / 2}\right),
$$

and taking $t=\operatorname{polylog}(n)$ the conclusion follows.

We also use the fact that the covariance matrix of a sum of sufficiently many gaussian outer products concentrates about its expectation.

Fact C.2. Let $a_{1}, \ldots, a_{n} \in \mathbb{R}^{d}$ be vectors with iid gaussian entries such that $\mathbb{E}\left[\left\|a_{i}\right\|_{2}^{2}\right]=1$, and $n=\Omega(d)$. Let $\mathcal{E}$ be the event that the sum $\sum_{i \in[n]} a_{i} a_{i}^{\top}$ is close to $\frac{n}{d} \cdot \mathrm{Id}$, that is

$$
\mathbb{P}\left\{\tilde{\Omega}(n / d) \cdot \mathrm{Id} \leqslant \sum_{i \in[n]} a_{i} a_{i}^{\top} \leqslant \tilde{O}(n / d) \cdot \operatorname{Id}\right\} \geqslant 1-n^{-\omega(1)} .
$$


Proof of Fact C.2. We apply a truncated matrix bernstein inequality. For convenience, $A:=$ $\sum_{i \in[n]} a_{i} a_{i}^{\top}$ and let $A_{i}:=a_{i} a_{i}^{\top}$ be a single summand. To begin, we calculate the first and second moments of the summands,

$$
\begin{aligned}
\mathbb{E}\left[A_{i}\right] & =\frac{1}{d} \cdot \mathrm{Id} \\
\mathbb{E}\left[A_{i} A_{i}^{\top}\right] & =O\left(\frac{1}{d}\right) \cdot \mathrm{Id} .
\end{aligned}
$$

So we have $\mathbb{E}[A]=\frac{n}{d} \cdot \operatorname{Id}$ and $\sigma^{2}(A)=O\left(\frac{n}{d}\right)$.

We now show that each summand is well-approximated by a truncated variable. To calculate the expected norm $\left\|A_{i}\right\|_{o p}$, we observe that $A_{i}$ is rank-1 and thus $\mathbb{E}\left[\left\|A_{i}\right\|_{o p}\right]=\mathbb{E}\left[\left\|a_{i}\right\|_{2}^{2}\right]=1$. Applying Lemma A.8, we have

$$
\mathbb{P}\left(\left\|A_{i}\right\|_{o p} \geqslant \tilde{O}(1)\right) \leqslant n^{-\log n},
$$

and also

$$
\mathbb{E}\left[\left\|A_{i}\right\|_{o p} \cdot \mathbb{I}\left\{\left\|A_{i}\right\|_{o p} \geqslant \tilde{O}(1)\right\}\right] \leqslant n^{-\log n} .
$$

Thus, applying the truncated matrix bernstein inequality from Proposition A.7 with $\sigma^{2}=O\left(\frac{n}{d}\right)$, $\beta=\tilde{O}(1), p=n^{-\log n}, q=n^{-\log n}$, and $t=\tilde{O}\left(\frac{n^{1 / 2}}{d^{1 / 2}}\right)$, we have that with overwhelming probability,

$$
\left\|A-\frac{n}{d} \cdot \mathrm{Id}\right\|_{o p} \leqslant \tilde{O}\left(\frac{n^{1 / 2}}{d^{1 / 2}}\right)
$$

We now show that among the terms of the polynomial $\left\langle g, T a_{i}^{\otimes 2}\right\rangle$, those that depend on $a_{j}$ with $j \neq i$ have small magnitude. This polynomial appears in the proof that $M_{\text {diag }}$ has a noticeable spectral gap.

Lemma (Restatement of Lemma 5.6). Let $a_{1}, \ldots, a_{n}$ be independently sampled vectors from $\mathcal{N}\left(0, \frac{1}{d} \operatorname{Id}_{d}\right)$, and let $g$ be sampled from $\mathcal{N}\left(0, \mathrm{Id}_{d}\right)$. Let $T=\sum_{i} a_{i}\left(a_{i} \otimes a_{i}\right)^{\top}$. Then with overwhelming probability, for every $j \in[n]$,

$$
\left|\left\langle g, T\left(a_{j} \otimes a_{j}\right)\right\rangle-\left\langle g, a_{j}\right\rangle\left\|a_{j}\right\|^{4}\right| \leqslant \tilde{O}\left(\frac{\sqrt{n}}{d}\right)
$$

Proof. Fixing $a_{i}$ and $g$, the terms in the summation are independent, and we may apply a Bernstein inequality. A straightforward calculation shows that the expectation of the sum is 0 and the variance is $\tilde{O}\left(\frac{n}{d^{2}}\right) \cdot\|g\|^{2}\left\|a_{i}\right\|^{4}$. Additionally, each summand is a polynomial in Gaussian variables, the square of which has expectation $\tilde{O}\left(\frac{1}{d^{2}} \cdot\|g\|^{2}\left\|a_{i}\right\|^{4}\right)$. Thus Lemma A.5 allows us to truncate each summand appropriately so as to employ Proposition A.7. An appropriate choice of logarithmic factors and the concentration of $\|g\|^{2}$ and $\left\|a_{i}\right\|^{2}$ due to Fact C.1 gives the result for each $i \in[n]$. A union bound over each choice of $i$ gives the final result.

Finally, we prove that a matrix which appears in the expression for $M_{\text {same }}$ has bounded norm w.ov.p. 
Lemma C.3. Let $a_{1}, \ldots, a_{n}$ be independent from $\mathcal{N}\left(0, \frac{1}{d} \operatorname{Id}_{d}\right)$. Let $g \sim \mathcal{N}\left(0, \operatorname{Id}_{d}\right)$. Fix $j \in[n]$. Then w.ov.p.

$$
\left\|\sum_{\substack{i \in[n] \\ i \neq j}}\left\langle g, a_{i}\right\rangle\right\| a_{i}\left\|^{2}\left\langle a_{i}, a_{j}\right\rangle \cdot a_{i} a_{i}^{\top}\right\| \leqslant \tilde{O}\left(n / d^{2}\right)^{1 / 2} .
$$

Proof. The proof proceeds by truncated matrix Bernstein, since the summands are independent for fixed $g, a_{j}$. For this we need to compute the variance:

$$
\sigma^{2}=\left\|\sum_{\substack{i \in[n] \\ i \neq j}} \mathbb{E}\left\langle g, a_{i}\right\rangle^{2}\right\| a_{i}\left\|^{6}\left\langle a_{i}, a_{j}\right\rangle^{2} \cdot a_{i} a_{i}^{\top}\right\| \leqslant O(1 / d) \cdot\left\|\sum_{\substack{i \in[n] \\ i \neq j}} \mathbb{E} a_{i} a_{i}^{\top}\right\| \leqslant O(1 / d) \cdot n / d \leqslant O\left(n / d^{2}\right) .
$$

The norm of each term in the sum is bounded by a constant-degree polynomial of Gaussians. Straightforward calculations show that in expectation each term is $O\left(\frac{1}{d}\left\langle g, a_{i}\right\rangle\right)$ in norm; w.ov.p. this is $O(\sigma)$. So Lemma A.5 applies to establish the hypothesis of truncated Bernstein Proposition A.7. In turn, Proposition A.7 yields that w.ov.p.

$$
\left\|\sum_{\substack{i \in[n] \\ i \neq j}}\left\langle g, a_{i}\right\rangle\right\| a_{i}\left\|^{2}\left\langle a_{i}, a_{j}\right\rangle \cdot a_{i} a_{i}^{\top}\right\| \leqslant \tilde{O}(\sigma)=\tilde{O}\left(n / d^{2}\right)^{1 / 2} .
$$

\section{C.0.3 Proof of Fact C.4}

Here we prove the following fact.

Fact C.4. Let $\Sigma=\mathbb{E}_{x \sim \mathcal{N}\left(0, \mathrm{Id}_{d}\right)}\left(x x^{\top}\right)^{\otimes 2}$ and let $\tilde{\Sigma}=\mathbb{E}_{x \sim \mathcal{N}\left(0, \mathrm{Id}_{d}\right)}\left(x x^{\top}\right)^{\otimes 2} /\|x\|^{4}$. Let $\Phi=\sum_{i} e_{i}^{\otimes 2} \in \mathbb{R}^{d^{2}}$ and let $\Pi_{\text {sym }}$ be the projector to the symmetric subspace of $\mathbb{R}^{d^{2}}$ (the span of vectors of the form $x^{\otimes 2}$ for $x \in \mathbb{R}^{d}$ ). Then

$$
\begin{array}{ll}
\Sigma=2 \Pi_{\mathrm{sym}}+\Phi \Phi^{\top}, & \tilde{\Sigma}=\frac{2}{d^{2}+2 d} \Pi_{\mathrm{sym}}+\frac{1}{d^{2}+2 d} \Phi \Phi^{\top}, \\
\Sigma^{+}=\frac{1}{2} \Pi_{\mathrm{sym}}-\frac{1}{2(d+2)} \Phi \Phi^{\top}, & \tilde{\Sigma}^{+}=\frac{d^{2}+2 d}{2} \Pi_{\mathrm{sym}}-\frac{d}{2} \Phi \Phi^{\top} .
\end{array}
$$

In particular,

$$
R=\sqrt{2}\left(\Sigma^{+}\right)^{1 / 2}=\Pi_{\text {sym }}-\frac{1}{d}\left(1-\sqrt{\frac{2}{d+2}}\right) \Phi \Phi^{\top} \text { has }\|R\|=1
$$

and for any $v \in \mathbb{R}^{d}$,

$$
\|R(v \otimes v)\|_{2}^{2}=\left(1-\frac{1}{d+2}\right) \cdot\|v\|^{4} .
$$

We will derive Fact C. 4 as a corollary of a more general claim about rotationally symmetric distributions. 
Lemma C.5. Let $\mathcal{D}$ be a distribution over $\mathbb{R}^{d}$ which is rotationally symmetric; that is, for any rotation $R$, $x \sim \mathcal{D}$ is distributed identically to $R x$. Let $\Sigma=\mathbb{E}_{x \sim \mathcal{D}}\left(x x^{\top}\right)^{\otimes 2}$, let $\Phi=\sum_{i} e_{i}^{\otimes 2} \in \mathbb{R}^{d^{2}}$ and let $\Pi_{\text {sym }}$ be the projector to the symmetric subspace of $\mathbb{R}^{d^{2}}$ (the span of vectors of the form $x^{\otimes 2}$ for $x \in \mathbb{R}^{d}$ ). Then there is a constant $r$ so that

$$
\Sigma=2 r \Pi_{\mathrm{sym}}+r \Phi \Phi^{\top}
$$

Furthermore, $r$ is given by

$$
r=\mathbb{E}\langle x, a\rangle^{2}\langle x, b\rangle^{2}=\frac{1}{3} \mathbb{E}\langle x, a\rangle^{4}
$$

where $a, b$ are orthogonal unit vectors.

Proof. First, $\Sigma$ is symmetric and operates nontrivially only on the symmetric subspace (in other words $\operatorname{ker} \Pi_{\text {sym }} \subseteq \operatorname{ker} \Sigma$ ). This follows from $\Sigma$ being an expectation over symmetric matrices whose kernels always contain the complement of the symmetric subspace.

Let $\hat{a}, \hat{b}, \hat{c}, \hat{d} \in \mathbb{R}^{d}$ be any four orthogonal unit vectors. Let $R$ be any rotation of $\mathbb{R}^{d}$ that takes $\hat{a}$ to $-\hat{a}$, but fixes $\hat{b}, \hat{c}$, and $\hat{d}$ (this rotation exists for $d \geqslant 5$, but a different argument holds for $d \leqslant 4$ ). By rotational symmetry about $R$, all of these quantities are 0 :

$$
\begin{gathered}
\mathbb{E}\langle\hat{a}, x\rangle\langle\hat{b}, x\rangle\langle\hat{c}, x\rangle\langle\hat{d}, x\rangle=0, \\
\mathbb{E}\langle\hat{a}, x\rangle\langle\hat{b}, x\rangle\langle\hat{c}, x\rangle^{2}=0, \quad \mathbb{E}\langle\hat{a}, x\rangle\langle\hat{b}, x\rangle^{3}=0 .
\end{gathered}
$$

Furthermore, let $Q$ be a rotation of $\mathbb{R}^{d}$ that takes $\hat{a}$ to $(\hat{a}+\hat{b}) / \sqrt{2}$. Then by rotational symmetry about $Q$,

$$
\mathbb{E}\langle\hat{a}, x\rangle^{4}=\mathbb{E}\langle\hat{a}, Q x\rangle^{4}=\mathbb{E} \frac{1}{4}\langle\hat{a}+\hat{b}, x\rangle^{4}=\mathbb{E} \frac{1}{4}\left[\langle\hat{a}, x\rangle^{4}+\langle\hat{b}, x\rangle^{4}+6\langle\hat{a}, x\rangle^{2}\langle\hat{b}, x\rangle^{2}\right]
$$

Thus, since $\mathbb{E}\langle\hat{a}, x\rangle^{4}=\mathbb{E}\langle\hat{b}, x\rangle^{4}$ by rotational symmetry, we have

$$
\mathbb{E}\langle\hat{a}, x\rangle^{4}=3 \mathbb{E}\langle\hat{a}, x\rangle^{2}\langle\hat{b}, x\rangle^{2} .
$$

So let $r:=\mathbb{E}\langle\hat{a}, x\rangle^{2}\langle\hat{b}, x\rangle^{2}=\frac{1}{3} \mathbb{E}\langle\hat{a}, x\rangle^{4}$. By rotational symmetry, $r$ is constant over choice of orthogonal unit vectors $\hat{a}$ and $\hat{b}$.

Since $\Sigma$ operates only on the symmetric subspace, let $u \in \mathbb{R}^{d^{2}}$ be any unit vector in the symmetric subspace. Such a $u$ unfolds to a symmetric matrix in $\mathbb{R}^{d \times d}$, so that it has an eigendecomposition $u=\sum_{i=1}^{d} \lambda_{i} u_{i} \otimes u_{i}$. Evaluating $\langle u, \Sigma u\rangle$,

$$
\begin{aligned}
\langle u, \Sigma u\rangle & =\sum_{i, j=1}^{d} \mathbb{E} \lambda_{i} \lambda_{j}\left\langle x, u_{i}\right\rangle^{2}\left\langle x, u_{j}\right\rangle^{2} \quad \text { other terms are } 0 \text { by above } \\
& =3 r \sum_{i=1}^{d} \lambda_{i}^{2}+r \sum_{i \neq j} \lambda_{i} \lambda_{j} \\
& =2 r \sum_{i=1}^{d} \lambda_{i}^{2}+r\left(\sum_{i=1}^{d} \lambda_{i}\right)^{2} \\
& =2 r\|u\|^{2}+r\left(\sum_{i=1}^{d} \lambda_{i}\right)^{2} \quad \text { Frobenious norm is sum of squared eigenvalues }
\end{aligned}
$$




$$
\begin{aligned}
& =2 r\|u\|^{2}+r\left(\sum_{i} u_{i, i}\right)^{2} \quad \text { trace is sum of eigenvalues } \\
& =2 r\left\langle u, \Pi_{\text {sym }} u\right\rangle+r\left\langle u, \Phi \Phi^{\top} u\right\rangle,
\end{aligned}
$$

so therefore $\Sigma=2 r \Pi_{\text {sym }}+r \Phi \Phi^{\top}$.

Proof of Fact C.4. When $x \sim \mathcal{N}\left(0, \mathrm{Id}_{d}\right)$, the expectation $\mathbb{E}\langle x, a\rangle^{2}\langle x, b\rangle^{2}=1$ is just a product of independent standard Gaussian second moments. Therefore by Lemma C.5, $\Sigma=2 \Pi_{\text {sym }}+\Phi \Phi^{\top}$.

To find $\tilde{\Sigma}$ where $x$ is uniformly distributed on the unit sphere, we compute

$$
1=\mathbb{E}\|x\|^{4}=\sum_{i, j} \mathbb{E} x_{i}^{2} x_{j}^{2}=d \mathbb{E} x_{1}^{4}+\left(d^{2}-d\right) \mathbb{E} x_{1}^{2} x_{2}^{2}
$$

and use the fact that $\mathbb{E} x_{1}^{4}=3 \mathbb{E} x_{1}^{2}$ (by Lemma C.5) to find that $\mathbb{E} x_{1}^{2} x_{2}^{2}=\frac{1}{d^{2}+2 d}$, and therefore by Lemma C.5, $\tilde{\Sigma}=\frac{2}{d^{2}+2 d} \Pi_{\text {sym }}+\frac{1}{d^{2}+2 d} \Phi \Phi^{\top}$.

To verify the pseudoinverses, it is enough to check that $M M^{+}=\Pi_{\text {sym }}$ for each matrix $M$ and its claimed pseudoinverse $\mathrm{M}^{+}$.

To show that

$$
\|R(v \otimes v)\|_{2}^{2}=\left(1-\frac{1}{d+2}\right) \cdot\|v\|^{4},
$$

for any $v \in \mathbb{R}^{d}$, we write $\|R(v \otimes v)\|_{2}^{2}=(v \otimes v)^{\top} R^{2}(v \otimes v)$ and use the substitution $R^{2}=2 \Sigma^{+}$, along with the facts that $\Pi_{\text {sym }}(v \otimes v)=v \otimes v$ and $\langle\Phi, v \otimes v\rangle=\|v\|^{2}$.

Now we can prove some concentration claims we deferred:

Lemma (Restatement of Lemma 5.11). Let $a_{1}, \ldots, a_{n} \sim \mathcal{N}\left(0, \frac{1}{d} \operatorname{Id}_{d}\right)$. Let $\Sigma, R$ be as in Fact 5.8. Let $u_{i}=a_{i} \otimes a_{i}$. With overwhelming probability, every $j \in[n]$ satisfies $\sum_{i \neq j}\left\langle u_{j}, R^{2} u_{i}\right\rangle^{2}=\tilde{O}\left(n / d^{2}\right)$ and $\left|1-\left\|R u_{j}\right\|^{2}\right| \leqslant \tilde{O}(1 / \sqrt{d})$.

Proof of Lemma 5.11. We prove the first item:

$$
\begin{aligned}
\sum_{i \neq j}\left\langle u_{j}, R^{2} u_{i}\right\rangle^{2} & =\sum_{i \neq j}\left\langle u_{j}, 2 \Sigma^{+} u_{i}\right\rangle^{2} \\
& =\sum_{i \neq j}\left\langle u_{j},\left(\Pi_{\text {sym }}-\frac{1}{d+2} \Phi \Phi^{\top}\right) u_{i}\right\rangle^{2} \quad \text { by Fact C. } 4 \\
& =\sum_{i \neq j}\left(\left\langle a_{j}, a_{i}\right\rangle^{2}-\frac{1}{d+2}\left\|u_{j}\right\|^{2}\left\|u_{i}\right\|^{2}\right)^{2} \\
& =\sum_{i \neq j} \tilde{O}(1 / d)^{2} \quad \text { w.ov.p. by Fact C.1 } \\
& =\tilde{O}\left(n / d^{2}\right) .
\end{aligned}
$$

And one direction of the second item, using Fact C.4 and Fact C.1 (the other direction is similar):

$$
\left\|R u_{j}\right\|^{2}=\left\langle u_{j}, R^{2} u_{j}\right\rangle=\left\langle u_{j},\left(\Pi_{\text {sym }}+\frac{1}{d+2} \Phi \Phi^{\top}\right) u_{j}\right\rangle=(1-\Theta(1 / d))\left\|a_{j}\right\|^{4}=1-\tilde{O}(1 / \sqrt{d})
$$

where the last equality holds w.ov.p.. 


\section{C.0.4 Proof of Lemma 5.9}

To prove Lemma 5.9 we will begin by reducing to the case $S=[n]$ via the following.

Lemma C.6. Let $v_{1}, \ldots, v_{n} \in \mathbb{R}^{d}$. Let $A_{S}$ have columns $\left\{v_{i}\right\}_{i \in S}$. Let $\Pi_{S}$ be the projector to Span $\left\{v_{i}\right\}_{i \in S}$. Suppose there is $c \geqslant 0$ so that $\left\|A_{[n]}^{\top} A_{[n]}-\operatorname{Id}_{n}\right\| \leqslant c$. Then for every $S \subseteq[n],\left\|A_{S} A_{S}^{\top}-\Pi_{S}\right\| \leqslant c$

Proof. If the hypothesized bound $\left\|A_{[n]}^{\top} A_{[n]}-\operatorname{Id}_{n}\right\| \leqslant c$ holds then for every $S \subseteq[n]$ we get $\| A_{S}^{\top} A_{S}-$ $\operatorname{Id}_{|S|} \| \leqslant c$ since $A_{S}^{\top} A_{S}$ is a principal submatrix of $A_{[n]}^{\top} A_{[n]}$. If $\left\|A_{S}^{\top} A_{S}-\operatorname{Id}_{|S|}\right\| \leqslant c$, then because $A_{S} A_{S}^{\top}$ has the same nonzero eigenvalues as $A_{S}^{\top} A_{S}$, we must have also $\left\|A_{S} A_{S}^{\top}-\Pi_{S}\right\| \leqslant c$.

It will be convenient to reduce concentration for matrices involving $a_{i} \otimes a_{i}$ to analogous matrices where the vectors $a_{i} \otimes a_{i}$ are replaced by isotropic vectors of constant norm. The following lemma shows how to do this.

Lemma C.7. Let $a \sim \mathcal{N}\left(0, \frac{1}{d} \operatorname{Id}_{d}\right)$. Let $\tilde{\Sigma}:=\mathbb{E}_{x \sim \mathcal{N}\left(0, \mathrm{Id}_{d}\right)}\left(x x^{\top}\right)^{\otimes 2} /\|x\|^{4}$. Then $u:=\left(\tilde{\Sigma}^{+}\right)^{1 / 2} a \otimes a /\|a\|^{2}$ is an isotropic random vector in the symmetric subspace $\operatorname{Span}\left\{y \otimes y \mid y \in \mathbb{R}^{d}\right\}$ with $\|u\|=$ $\sqrt{\operatorname{dim} \operatorname{Span}\left\{y \otimes y \mid y \in \mathbb{R}^{d}\right\}}$.

Proof. The vector $u$ is isotropic by definition so we prove the norm claim. Let $\tilde{\Phi}=\Phi /\|\Phi\|$. By Fact C.4,

$$
\tilde{\Sigma}^{+}=\frac{d^{2}+2 d}{2} \Pi_{\text {sym }}-\frac{d}{2} \Phi \Phi^{\top}
$$

Thus,

$$
\|u\|^{2}=\left\langle\frac{a \otimes a}{\|a\|^{2}}, \tilde{\Sigma}^{+} \frac{a \otimes a}{\|a\|^{2}}\right\rangle=\frac{d^{2}+2 d}{2}-\frac{d}{2}=\frac{d^{2}+d}{2}=\operatorname{dim} \operatorname{Span}\left\{y \otimes y \mid y \in \mathbb{R}^{d}\right\} .
$$

The last ingredient to finish the spectral bound is a bound on the incoherence of independent samples from $\left(\tilde{\Sigma}^{+}\right)^{1 / 2}$.

Lemma C.8. Let $\tilde{\Sigma}=\mathbb{E}_{a \sim \mathcal{N}\left(0, \operatorname{Id}_{d}\right)}\left(a a^{\top} \otimes a a^{\top}\right) /\|a\|^{4}$. Let $a_{1}, \ldots, a_{n} \sim \mathcal{N}\left(0, \operatorname{Id}_{d}\right)$ be independent, and let $u_{i}=\left(\tilde{\Sigma}^{+}\right)^{1 / 2}\left(a_{i} \otimes a_{i}\right) /\left\|a_{i}\right\|^{2}$. Let $d^{\prime}=\operatorname{dim} \operatorname{Span}\left\{y \otimes y \mid y \in \mathbb{R}^{d}\right\}=\frac{1}{2}\left(d^{2}+d\right)$. Then

$$
\frac{1}{d^{\prime}} \mathbb{E} \max _{i} \sum_{j \neq i}\left\langle u_{i}, u_{j}\right\rangle^{2} \leqslant \tilde{O}(n) .
$$

Proof. Expanding $\left\langle u_{i}, u_{j}\right\rangle^{2}$ and using $\tilde{\Sigma}^{+}=\frac{d^{2}+2 d}{2} \prod_{\text {sym }}-\frac{d}{2} \Phi \Phi^{\top}$, we get

$$
\left\langle u_{i}, u_{j}\right\rangle^{2}=\left(\frac{d^{2}+2 d}{2}\left\langle\frac{a_{i} \otimes a_{i}}{\left\|a_{i}\right\|^{2}}, \frac{a_{j} \otimes a_{j}}{\left\|a_{j}\right\|^{2}}\right\rangle-\frac{d}{2}\right)^{2}=\left(\frac{d^{2}+2 d}{2} \cdot \frac{\left\langle a_{i}, a_{j}\right\rangle^{2}}{\left\|a_{i}\right\|^{2}\left\|a_{j}\right\|^{2}}-\frac{d}{2}\right)^{2}
$$

From elementary concentration, $\mathbb{E} \max _{i \neq j}\left\langle a_{i}, a_{j}\right\rangle^{2} /\left\|a_{i}\right\|^{2}\left\|a_{j}\right\|^{2} \leqslant \tilde{O}(1 / d)$, so the lemma follows by elementary manipulations.

We need the following bound on the deviation from expectation of a tall matrix with independent columns.

Theorem C.9 (Theorem 5.62 in [Ver10]). Let $A$ be an $N \times n$ matrix $(N \geqslant n)$ whose columns $A_{j}$ are independent isotropic random vectors in $\mathbb{R}^{N}$ with $\left\|A_{j}\right\|_{2}=\sqrt{N}$ almost surely. Consider the incoherence parameter

$$
m \stackrel{\text { def }}{=} \frac{1}{N} \mathbb{E} \max _{i \in[n]} \sum_{j \neq i}\left\langle A_{i}, A_{j}\right\rangle^{2} .
$$

Then $\mathbb{E}\left\|\frac{1}{N} A^{T} A-\operatorname{Id}\right\| \leqslant C_{0} \sqrt{\frac{m \log n}{N}}$. 
We are now prepared to handle the case of $S=[n]$ via spectral concentration for matrices with independent columns, Theorem C.9.

Lemma (Restatement of Lemma 5.9). Let $a_{1}, \ldots, a_{n} \sim \mathcal{N}\left(0, \frac{1}{d} \mathrm{Id}_{d}\right)$ be independent random vectors with $d \leqslant n$. Let $R:=\sqrt{2} \cdot\left(\left(\mathbb{E}\left(a a^{\top}\right)^{\otimes 2}\right)^{+}\right)^{1 / 2}$ for $a \sim \mathcal{N}\left(0, \operatorname{Id}_{d}\right)$. For $S \subseteq[n]$, let $P_{S}=\sum_{i \in S}\left(a_{i} a_{i}^{\top}\right)^{\otimes 2}$ and let $\Pi_{S}$ be the projector into the subspace spanned by $\left\{R a_{i}^{\otimes 2} \mid i \in S\right\}$. Then, with probability $1-o(1)$ over the choice of $a_{1}, \ldots, a_{n}$,

$$
\forall S \subseteq[n] . \quad\left(1-\tilde{O}\left(n / d^{3 / 2}\right)\right) \cdot \Pi_{S} \leq R P_{S} R \leq\left(1+\tilde{O}\left(n / d^{3 / 2}\right)\right) \cdot \Pi_{S} .
$$

Proof of Lemma 5.9. By Lemma C.6 it is enough to prove the lemma in the case of $S=[n]$. For this we will use Theorem C.9. Let $A$ be the matrix whose columns are given by $a_{i} \otimes a_{i}$, so that $P_{[n]}=P=A A^{\top}$. Because $R A A^{\top} R$ and $A^{\top} R R A$ have the same nonzero eigenvalues, it will be enough to show that $\left\|A^{\top} R^{2} A-\operatorname{Id}\right\| \leqslant \tilde{O}(\sqrt{n} / d)+\tilde{O}\left(n / d^{3 / 2}\right)$ with probability $1-o(1)$. (Since $n \leqslant d$ we have $\sqrt{n} / d=\tilde{O}\left(n / d^{3 / 2}\right)$ so this gives the theorem.)

The columns of $R A$ are independent, given by $R\left(a_{i} \otimes a_{i}\right)$. However, they do not quite satisfy the normalization conditions needed for Theorem C.9. Let $D$ be the diagonal matrix whose $i$-th diagonal entry is $\left\|a_{i}\right\|^{2}$. Let $\tilde{\Sigma}=\mathbb{E}_{x \sim \mathcal{N}(0, \mathrm{Id})}\left(x x^{\top}\right)^{\otimes 2} /\|x\|^{4}$. Then by Lemma C.7 the matrix $\left(\tilde{\Sigma}^{+}\right)^{1 / 2} D^{-1} A$ has independent columns from an isotropic distribution with a fixed norm $d^{\prime}$. Together with Lemma C.8 this is enough to apply Theorem C.9 to conclude that $\mathbb{E}\left\|\frac{1}{\left(d^{\prime}\right)^{2}} A^{\top} D^{-1} \tilde{\Sigma}^{+} D^{-1} A-\operatorname{Id}\right\| \leqslant$ $\tilde{O}(\sqrt{n} / d)$. By Markov's inequality, $\left\|\frac{1}{\left(d^{\prime}\right)^{2}} A^{\top} D^{-1} \tilde{\Sigma}^{+} D^{-1} A-\operatorname{Id}\right\| \leqslant \tilde{O}(\sqrt{n} / d)$ with probability $1-o(1)$.

We will show next that $\left\|A^{\top} R^{2} A-\frac{1}{\left(d^{\prime}\right)^{2}} A^{\top} D^{-1} \tilde{\Sigma}^{+} D^{-1} A\right\| \leqslant \tilde{O}\left(n / d^{3 / 2}\right)$ with probability $1-o(1)$; the lemma then follows by triangle inequality. The expression inside the norm expands as

$$
A^{\top}\left(R^{2}-\frac{1}{\left(d^{\prime}\right)^{2}} D^{-1} \tilde{\Sigma}^{+} D^{-1}\right) A
$$

and so

$$
\left\|A^{\top} R^{2} A-\frac{1}{\left(d^{\prime}\right)^{2}} A^{\top} D^{-1} \tilde{\Sigma}^{+} D^{-1} A\right\| \leqslant\|A\|^{2}\left\|R^{2}-\frac{1}{\left(d^{\prime}\right)^{2}} D^{-1} \tilde{\Sigma}^{+} D^{-1}\right\|
$$

By Fact C.1, with overwhelming probability $\|D-\mathrm{Id}\| \leqslant \tilde{O}(1 / \sqrt{d})$. So $\|\left(1 / d^{\prime}\right)^{2} D^{-1} \tilde{\Sigma}^{+} D^{-1}-$ $\left(1 / d^{\prime}\right)^{2} \tilde{\Sigma}^{+} \| \leqslant \tilde{O}(1 / \sqrt{d})$ w.ov.p.. We recall from Fact C.4, given that $R=\sqrt{2} \cdot\left(\Sigma^{+}\right)^{1 / 2}$, that

$$
R^{2}=\Pi_{\mathrm{sym}}-\frac{1}{d+2} \Phi \Phi^{\top} \quad \text { and } \quad \frac{1}{\left(d^{\prime}\right)^{2}} \tilde{\Sigma}^{+}=\frac{d+2}{d+1} \Pi_{\mathrm{sym}}-\frac{1}{d+1} \Phi \Phi^{\top}
$$

This implies that $\left\|R^{2}-\left(1 / d^{\prime}\right)^{2} \tilde{\Sigma}^{+}\right\| \leqslant O(1 / d)$. Finally, by an easy application of Proposition A.7, $\|A\|^{2}=\left\|\sum_{i}\left(a_{i} a_{i}^{\top}\right)^{\otimes 2}\right\| \leqslant \tilde{O}(n / d)$ w.ov.p.. All together, $\left\|A^{\top} R^{2} A-\frac{1}{\left(d^{\prime}\right)^{2}} A^{\top} D^{-1} \tilde{\Sigma}^{+} D^{-1} A\right\| \leqslant \tilde{O}\left(n / d^{3 / 2}\right)$.

\section{Concentration bounds for tensor principal component analysis}

For convenience, we restate Lemma 6.5 here.

Lemma D.1 (Restatement of Lemma 6.5). For any v, with high probability over A, the following occur:

$$
\begin{aligned}
\left\|\sum_{i} \operatorname{Tr}\left(A_{i}\right) \cdot A_{i}\right\| & \leqslant O\left(n^{3 / 2} \log ^{2} n\right) \\
\left\|\sum_{i} v(i) \cdot A_{i}\right\| & \leqslant O(\sqrt{n} \log n)
\end{aligned}
$$




$$
\left\|\sum_{i} \operatorname{Tr}\left(A_{i}\right) v(i) \cdot v v^{T}\right\| \leqslant O(\sqrt{n} \log n) .
$$

Proof of Lemma 6.5. We begin with the term $\sum_{i} \operatorname{Tr}\left(A_{i}\right) \cdot A_{i}$. It is a sum of iid matrices $\operatorname{Tr}\left(A_{i}\right) \cdot A_{i}$. A routine computation gives $\mathbb{E} \operatorname{Tr}\left(A_{i}\right) \cdot A_{i}=\mathrm{Id}$. We will use the truncated matrix Bernstein's inequality (Proposition A.7) to bound $\left\|\sum_{i} \operatorname{Tr}\left(A_{i}\right) A_{i}\right\|$.

For notational convenience, let $A$ be distributed like a generic $A_{i}$. By a union bound, we have both of the following:

$$
\begin{array}{r}
\mathbb{P}(\|\operatorname{Tr}(A) \cdot A\| \geqslant t n) \leqslant \mathbb{P}(|\operatorname{Tr}(A)| \geqslant \sqrt{t n})+\mathbb{P}(\|A\| \geqslant \sqrt{t n}) \\
\mathbb{P}(\|\operatorname{Tr}(A) \cdot A-\operatorname{Id}\| \geqslant(t+1) n) \leqslant \mathbb{P}(|\operatorname{Tr}(A)| \geqslant \sqrt{t n})+\mathbb{P}(\|A\| \geqslant \sqrt{t n}) .
\end{array}
$$

Since $\operatorname{Tr}(A)$ the sum of iid Gaussians, $\mathbb{P}(|\operatorname{Tr}(A)| \geqslant \sqrt{t n}) \leqslant e^{-c_{1} t}$ for some constant $c_{1}$. Similarly, since the maximum eigenvalue of a matrix with iid entries has a subgaussian tail, $\mathbb{P}(\|A\| \geqslant \sqrt{t n}) \leqslant$ $e^{-c_{2} t}$ for some $c_{2}$. All together, for some $c_{3}$, we get $\mathbb{P}(\|\operatorname{Tr}(A) \cdot A\| \geqslant t n) \leqslant e^{-c_{3} t}$ and $\mathbb{P}(\|\operatorname{Tr}(A) \cdot A-\operatorname{Id}\| \geqslant$ $(t+1) n) \leqslant e^{-c_{3} t}$.

For a positive parameter $\beta$, let $\mathbb{I}_{\beta}$ be the indicator variable for the event $\|\operatorname{Tr}(A) \cdot A\| \leqslant \beta$. Then

$$
\begin{aligned}
\mathbb{E}\|\operatorname{Tr}(A) \cdot A\|-\mathbb{E}\|\operatorname{Tr}(A) \cdot A\| \mathbb{I}_{\beta} & =\int_{0}^{\infty}\left[\mathbb{P}(\|\operatorname{Tr} A \cdot A\|>s)-\mathbb{P}\left(\|\operatorname{Tr} A \cdot A\| \mathbb{I}_{\beta}>s\right)\right] \mathrm{d} s \\
& =\beta \mathbb{P}(\|\operatorname{Tr} A \cdot A\|>\beta)+\int_{\beta}^{\infty} \mathbb{P}(\|\operatorname{Tr} A \cdot A\|>s) \mathrm{d} s \\
& \leqslant \beta e^{-c_{3} \beta / n}+\int_{\beta}^{\infty} \mathbb{P}(\|\operatorname{Tr} A \cdot A\|>s) \mathrm{d} s \\
& =\beta e^{-c_{3} \beta / n}+\int_{\beta / n}^{\infty} \mathbb{P}(\|\operatorname{Tr} A \cdot A\| \geqslant t n) n \mathrm{~d} t \\
& \leqslant \beta e^{-c_{3} \beta / n}+\int_{\beta / n}^{\infty} n e^{-c_{3} t} \mathrm{~d} t \\
& =\beta e^{-c_{3} \beta / n}+\frac{n}{c_{3}} e^{-c_{3} \beta / n} .
\end{aligned}
$$

Thus, for some $\beta=O(n \log n)$ we may take the parameters $p, q$ of Proposition A.7 to be $O\left(n^{-150}\right)$. The only thing that remains is to bound the parameter $\sigma^{2}$. Since $(\mathbb{E} \operatorname{Tr}(A) \cdot A)^{2}=\mathrm{Id}$, it is enough just to bound $\left\|\mathbb{E} \operatorname{Tr}(A)^{2} A A^{T}\right\|$. We use again a union bound:

$$
\mathbb{P}\left(\left\|\operatorname{Tr}(A)^{2} A A^{T}\right\|>t n^{2}\right) \leqslant \mathbb{P}\left(|\operatorname{Tr}(A)|>t^{1 / 4} \sqrt{n}\right)+\mathbb{P}\left(\|A\|>t^{1 / 4} \sqrt{n}\right) .
$$

By a similar argument as before, using the Gaussian tails of $\operatorname{Tr} A$ and $\|A\|$, we get $\mathbb{P}\left(\left\|\operatorname{Tr}(A)^{2} A A^{T}\right\|>\right.$ $\left.t n^{2}\right) \leqslant e^{-c_{4} \sqrt{t}}$. Then starting out with the triangle inequality,

$$
\begin{aligned}
\sigma^{2} & =\left\|n \cdot \mathbb{E} \operatorname{Tr}(A)^{2} A A^{T}\right\| \\
& \leqslant n \cdot \mathbb{E}\left\|\operatorname{Tr}(A)^{2} A A^{T}\right\| \\
& =n \cdot \int_{0}^{\infty} \mathbb{P}\left(\operatorname{Tr}(A)^{2} A A^{T}>s\right) \mathrm{d} s \\
& =n \cdot \int_{0}^{\infty} \mathbb{P}\left(\operatorname{Tr}(A)^{2} A A^{T}>t n^{2}\right) n^{2} \mathrm{~d} t
\end{aligned}
$$




$$
\begin{aligned}
& \leqslant n \cdot \int_{0}^{\infty} e^{-c_{4} \sqrt{t}} n^{2} \mathrm{~d} t \\
& =n \cdot\left[-\frac{2 n^{2}\left(c_{4} \sqrt{t}+1\right)}{c_{4}^{2}} e^{-c_{4} \sqrt{t}}\right]_{t=0}^{t=\infty} \\
& \leqslant O\left(n^{3}\right) .
\end{aligned}
$$

This gives that with high probability,

$$
\left\|\sum_{i} \operatorname{Tr}\left(A_{i}\right) \cdot A_{i}\right\| \leqslant O\left(n^{3 / 2} \log ^{2} n\right) .
$$

The other matrices are easier. First of all, we note that the matrix $\sum_{i} v(i) \cdot A_{i}$ has independent standard Gaussian entries, so it is standard that with high probability $\left\|\sum_{i} v(i) \cdot A_{i}\right\| \leqslant O(\sqrt{n} \log n)$. Second, we have

$$
\sum_{i} v(i) \operatorname{Tr}\left(A_{i}\right) v v^{T}=v v^{T} \sum_{i} v(i) \operatorname{Tr}\left(A_{i}\right)
$$

The random variable $\operatorname{Tr}\left(A_{i}\right)$ is a centered Gaussian with variance $n$, and since $v$ is a unit vector, $\sum_{i} v(i) \operatorname{Tr}\left(A_{i}\right)$ is also a centered Gaussian with variance $n$. So with high probability we get

$$
\left\|v v^{T} \sum_{i} v(i) \operatorname{Tr}\left(A_{i}\right)\right\|=\left|\sum_{i} v(i) \operatorname{Tr}\left(A_{i}\right)\right| \leqslant O(\sqrt{n} \log n)
$$

by standard estimates. This completes the proof. 